\title{
ConsEuro Conference 2015, London, May 14-16
}

The bi-annual ConsEuro Conference 2015 was recently held in London at the Queen Elizabeth II conference centre, Westminster. The conference was hosted by Professor Stephen Dunne, President of the European Federation of Conservative Dentistry (EFCD) and was a joint meeting of the EFCD, British Society for Restorative Dentistry, Association of Teachers of Conservative Dentistry and King's College London.

The theme of the conference was Technology in Dentistry and in keeping with that theme, the conference was as near paperless as possible. The conference proved a great success with over 500 delegates attending, from 29 different countries, from as far afield as India, Australia, Senegal, USA and, of course, from right across Europe.

Over 40 lecturers and demonstrators from around the world presented a truly international view and covered the full range of technologies in dentistry. The programme also included ancillary meetings, lectures and demonstrations including the Academy of Operative Dentistry, European Section, sponsored by DMG and separate meetings organised and sponsored by Kuraray, Colgate, Unilever, the BDJ, 3M ESPE and Henry Schein. The conference received extraordinary support from the dental industry, the Gold Sponsors were Colgate, 3M ESPE, Kuraray and Unilever. The Silver Sponsors were Dentsply, Dentinal Tubules, Septodont, and Ivoclar Vivadent, while the Bronze Sponsors were Henry Schein and Planmeca. Exhibitors included ARC Laser, Dentists Provident, GC, the BDJ, Quintessence Publishing and NS.

Professor Dunne expressed his gratitude to the dental industry for their generosity and also to the ConsEuro Conference Committee, in particular to Mr Leslie Howe, Professor Avi Banerji, and Mr Jim Lucas of Hampton Medical, our Professional Conference Organiser, for their tireless efforts in organising this conference.

ConsEuro 2017 will be held in Italy and the EFCD look forward to welcoming you.

Professor Stephen Dunne

President of the EFCD

\section{Biomaterials (BM)}

BM01

Laboratory evaluation of tooth dissolution in cough syrups

Cornelius Bamise, Temitope Esan, Elizabeth Oziegbe, Francis Alo Obafemi Awolowo University, Ile Ife, Osun State, Nigeria

Background: Currently, the increased consumption of acidic foods and medications is becoming an important factor for the development of erosive wear because of their acidic nature. Therefore, most clinical and laboratory researches have focused on their dental impact.

Aim. To evaluate the associated weight loss of extracted teeth after immersion in cough syrups for a period of time.

Materials and Methods. pHs of the medicines were measured at the start of the study.

25 extracted test teeth were weighed to $0.01 \mathrm{mg}$ and assigned at random to each syrup under evaluation. The test medicines were placed in $10 \mathrm{~mL}$ screw-cap plastic containers and the test teeth were weighed after 7 days.

Result. $88 \%$ of the medicines caused reduction in weights of the test teeth after seven days of immersion. Weight loss of the test teeth also noticed with basic syrups. Also lowest $\mathrm{pH}$ syrup did not produce the greatest weight loss.

Conclusion: Majority of the cough syrups caused loss of weight of the test teeth. Thus, they possess the potential to cause dental erosion.

\section{BM02}

Quantification of the amount of blue light passing through monolithic zirconia

Nicoleta Ilie ${ }^{1}$, Bogna Stawarczyk ${ }^{2}$

${ }^{T}$ Dental School, Ludwig-Maximilians University, 1Department of Operative/Restorative Dentistry, Periodontology and Pedodontics, Munich, Germany, ${ }^{2}$ Dental School, Ludwig-Maximilians University, 2Department of Prosthodontics, Munich, Germany

Dual-polymerized luting composite resin cements would benefit from an enhanced irradiance transmitted through a ceramic restoration. A quantification of the amount of transmitted light through translucent zirconia is missing so far. It was the purpose of this study to evaluate the amount of light (360 to $540 \mathrm{~nm}$ ) passing diverse ceramic materials with respect to material thickness and different polymerizing modes and to identify material parameters allowing calculation of the amount of transmitted irradiance at any initial irradiance or material thickness.

Six translucent and a conventional zirconia (negative control) as well as a glass-ceramic (positive control group) were considered. Ten specimens of each material and thickness $(0.5 ; 1 ; 1.5 ; 2 ; 2.5 ; 3 \mathrm{~mm})$ were fabricated $(\mathrm{N}$ 
$=480$ ). The irradiance passing the different ceramics and thicknesses was measured using a violet-blue LED polymerizing unit in three polymerizing modes with a USB4000 Spectrometer. Data were analyzed using one and multivariate analysis as well as Pearson correlation analysis $(\mathrm{p}<0.05)$.

Zirconia was less translucent compared to the glass-ceramic, but the translucency decreased more slowly with material thickness, thus approaching the translucency of glass-ceramics at specimen thicknesses of 2.5 to $3 \mathrm{~mm}$.

The decrease of transmitted irradiance with increased specimen's thickness was well described by the Beer-Lambert law in all materials. The derivative of this equation allows determination of the velocity of translucency decrease as a function of thickness. The determined equation parameters allow calculation of the amount of transmitted irradiance for the analysed materials at any initial irradiance or material thickness.

\section{BM03}

Effect of MTA setting time intervals on its shear bond strength with overlying restorative materials.

Ahmed Ali, Avijit Banerjee, Francesco Mannocci

King's College London, London, UK

Objective: in-vitro comparison of shear bond strength (SBS) and interface failure modes (IFM) between mineralised trioxide aggregate (MTA) and glass ionomer cement (GIC) or resin composite (RC) after different setting time intervals for MTA.

Materials and Method: MTA was mixed and packed into standardised holes $\left(4 \mathrm{X} 3 \mathrm{~mm}\right.$ ) in acrylic blocks. $\mathrm{RC}$ (with $37 \% \mathrm{H}_{3} \mathrm{PO}_{4}$ and type 2 adhesive used per manufacturer's instructions) or GIC were bonded to MTA specimens either after $10 \mathrm{~min}, 24 \mathrm{hrs}$ and $72 \mathrm{hrs}$ intervals $(\mathrm{n}=10$ / group, 6 groups). All groups were stored $\left(37 \mathrm{C}^{0}, 24 \mathrm{hrs}\right)$ before testing. SBS was tested $(0.5 \mathrm{~mm} / \mathrm{m}$ ) and statistically analysed (ANOVA, LSD, $p<$ 0.05 ). IFM were identified using stereomicroscopy and SEM images of three samples were analysed.

Results: There was a significant difference between all groups $(\mathrm{p}=$ 0.003). SBS of RC to MTA $(5.09 \pm 1.79 \mathrm{MPa})$ was higher than SBS of GIC to MTA $(2.77 \pm 1.05 \mathrm{MPa})$. There was significant increase in SBS of $\mathrm{RC}$ to MTA with time except in 10min setting time groups, where SBS of GIC to MTA was higher. IFM were mixed (adhesive and cohesive) in all groups but in RC-MTA groups there was decrease in adhesive trend with time in contrast to GIC-MTA groups.

Conclusion: Shear bond strengths between RC and MTA was significantly higher than that of GIC and MTA after 24 and 72 hours' time intervals of MTA setting time.

Clinical relevance: If a vital pulp therapy treatment in one session is to be considered it will be feasible to use GIC over unset MTA before placement of a RC.

\section{BM04}

Influence of tetracalcium-phosphate (TTCP) and calcium-phosphate acid (DCPA) on bond-strength of self-etch adhesives

Allegra Comba $^{1}$, Mattia Chiapello ${ }^{1}$, Lorenzo Breschi ${ }^{2}$, Gianluca Turco ${ }^{3}$, Elio Berutti ${ }^{1}$, Nicola Scotti ${ }^{1}$

${ }^{1}$ University of Turin, Turin, Italy, ${ }^{2}$ Alma Mater Studiorum, Bologna, Italy,

${ }^{3}$ University of Trieste, Trieste, Italy
Objective: The aim of this in-vitro study was to evaluate the influence of a dentinal pre-treatment with TTCP and DCPA on the bond-strength of 2 adhesive systems employed with the etchand-dry technique. The null hypothesis are that the application of synthetic hydroxyapatite over dentin (1) is not able to influence bond strength of adhesives applied following an etch-anddry approach and (2) it does not prevent adhesive interface degradation.

Methods: 60 molars crowns were flattened and standardized smear layer was created. Samples were randomly divided in 6 groups:

G1 2-step self-etch adhesive (Clearfil Protect Bond, Kuraray) application

G2 TeethMate Desensitizer (Kuraray), water rinse for 10 seconds and the 2-step self-etch adhesive application

G3 10\%EDTA for 30 seconds, air drying and TeethMate Desensitizer and 2-step adhesive application

G4 universal adhesive (All Bond Universal, Bisco) application

G5 TeethMate Desensitizer, water rinse for 10 seconds and universal adhesive application

G6 $10 \%$ EDTA for 30 seconds, air drying and TeethMate Desensitizer and universal adhesive application

Then resin composite was applied. Specimens were serially sectioned to obtain 1-mm-thick beams in accordance with the $\mu$ TBS test technique. Beams were stressed to failure after $24 \mathrm{~h}, 6$ and 12 months of storage in artificial buffer at $37 \mathrm{C}$.

Results: Three-way Anova showed that adhesive system, treatment protocol and aging are influencing factors $(\mathrm{p}<0.0001)$, but not their interactions.

Conclusion: Null hypothesis are partially refused since the application of TTCP and DCPA is able to improve bond strength values and degradation overtime only if employed in combination with dentin pre-treatment with $10 \%$ EDTA.

\section{BM05}

The effect of MMP-inhibitor modified adhesive primer on the caries-affected dentine: A Raman spectroscopy study

Ahmed Almahdy $^{1,2}$, Garrit Koller ${ }^{1}$, Frederic Festy ${ }^{1}$, Jörg Bartsch ${ }^{1,3}$, Timothy F. Watson', Avijit Banerjee ${ }^{1}$

${ }^{1}$ Dental Institute, King's College London, London, UK, ${ }^{2}$ College of Dentistry, King Saud University, Riyadh, Saudi Arabia, ${ }^{3}$ University of Marburg, Marburg, Germany

Objectives: To evaluate the effect of adding a matrix metalloproteinase (MMP) inhibitor (BB94) to the primer of a three-step etch and rinse adhesive system on the chemical content of the caries-affected dentine $(\mathrm{CaD})$-adhesive interface.

Methods: Caries-infected dentine (CiD) was excavated selectively from five freshly extracted human carious teeth using a chemo-mechanical agent. Each tooth was sectioned into three slabs. These were treated with either Optibond FL “OB” (Kerr, Orange, USA) without MMP inhibitor, or with $500 \mu \mathrm{M}$ BB94 prior to the application of OB primer and bond, or with OB primer that contained $5 \mu \mathrm{M}$ BB94. Raman micro-spectroscopy 
(Renishaw Plc, Wotton-under-Edge, UK) was used to investigate the changes in the chemical content at the $\mathrm{CaD}$-adhesive interface. The interface areas were scanned immediately (week 0), after two weeks (week 2) and after one month (week 4). Pearson-based cluster analysis of the dataset (over 1.8 million spectra) was performed. The average cluster content within the hybrid layer layer was calculated for all groups. Two-way ANOVA and Tukey's post-hoc test were used to compare the differences in the relative contribution of each cluster between the three groups at the three different times.

Results: The use of OB adhesive with BB94 resulted in 33\% higher resin infiltration into $\mathrm{CaD}$-adhesive interfaces (SE 3.88). This was maintained over time.

Significance: Through competitive inhibition by BB94, the adhesive primer was brought close to the collagen matrix and the dental adhesive wettability was enhanced. This proposed mechanism explains the presence of more resin within the hybrid layer.

\section{BM06}

Evaluation of hardness increase of GIC restorative surface in saliva.

Yusuke Shimada, Yusuke Hokii, Katsushi Yamamoto, Shouichi Fukushima, Tomohiro Kumagai

GC Corporation $R \&$ D centre, Tokyo, Japan

Objective: Increasing of surface hardness of GIC in saliva is one of unique phenomenon. The aim of this study was to evaluate the surface hardness of new experimental GI filling system.

Methods: Experimental system consists of experimental GI filling material, EFI-300 and experimental surface coating material, EQC-107. EFI300 and Fuji IX GP EXTRA (F9E) were filled into acrylic mold (7 $\mathrm{mm}$ in diameter, $2 \mathrm{~mm}$ in depth). Specimens were then stored at $37^{\circ} \mathrm{C}$, $100 \%$ R.H. for 1 hour. Specimens were then stored as follows under $37^{\circ} \mathrm{C}$, EWC: EFI-300 with EQC-107 in water. ESC: EFI-300 with EQC-107 in saliva. EW: EFI-300 in water. ES: EFI-300 in saliva. XWC: F9E with G-Coat Plus (GCP) in water. XSC: F9E with GCP in saliva. XW: F9E in water. ES: F9E in saliva. After 0, 30 and 65days, surface hardness of specimens were measured with micro vickers hardness machine (SHIMADZU: HMV-G21DT). Coatings were removed from GI surface before measuring vickers hardness. Specimens were recoated before immersion to storage from 0 to 30 days. After 30days, all specimens were immersed in the solutions without coatings.

Result: Results of 30days are ESC(121HV), XSC(106), EWC(117), XWC(126), ES(116), XS(115), EW(105), XW(106), and 65 days are ESC(141HV), XSC(139), EWC(118), XWC(116), ES(127), XS(129), $\mathrm{EW}(105), \mathrm{XW}(92)$.

Conclusion: These results indicate that new experimental GI filling system also increases in surface hardness in saliva.

\section{BM07}

\section{Comparison of Mechanical Properties of Composite with Different Enamel Shades}

Zeynep Bilge Kutuk ${ }^{1}$, Sevil Gurgan ${ }^{1}$, Reinhard Hickel ${ }^{2}$, Nicoleta Ilie ${ }^{2}$ ${ }^{T}$ Hacettepe University Faculty of Dentistry Restorative Dentistry Department, Ankara, Turkey, ${ }^{2}$ Ludwig Maximilians University, School of Dentistry, Department of Restorative Dentistry and Periodontology, Munich, Germany
Objective: To compare the macro- and micro-mechanical properties of a highly translucent composite with different enamel shades.

Materials and methods: Three different enamel shades of a highly translucent composite were used (G-aenial Anterior SE, AE and JE). The specimens $(\mathrm{n}=20)$ were prepared by a steel mould having an internal dimension of $2 \times 2 \times 16 \mathrm{~mm}$. Irradiation was occurred on top and at the bottom of the specimens, with three light exposures of $20 \mathrm{~s}$ per side. The specimens were ground with silicon carbide sand papers (1200/4000), then stored in distilled water at $37^{\circ} \mathrm{C}$ for $24 \mathrm{~h}$. The specimens were loaded until failure in the universal testing machine in a three-point bending test device to determine flexural strength and flexural modulus. Micromechanical properties -Vickers hardness (HV), indentation modulus $(\mathrm{E})$ and creep $(\mathrm{Cr})$ were examined by a universal hardness device. Six measurements per specimen, 60 measurements for each material were done. Results were statistically compared using one-way ANOVA and Tukey's HSD post hoc test $(\alpha=0.05)$.

Results: Among the three different enamel shades, SE which mimics elderly people enamel shade showed the lowest values for all mechanical properties. Conversely, JE shade, suitable for young patients, showed the highest flexural strength, flexural modulus, $\mathrm{E}$ and $\mathrm{Cr}$ values. Nevertheless, no significant differences were found between the macro- and micro-mechanical properties of the composites with different enamel shades $(p>0.05)$.

Conclusion: Differences in enamel shades did not affect the mechanical properties of highly translucent composites.

\section{BM08}

The Effect of Different Storage Mediums on Dentin Moisture, Microhardness and Bonding

Aysegul Kaya ${ }^{1}$, Tijen Pamir ${ }^{1}$, Pinar Kosker ${ }^{1}$, Bilge Hakan Sen ${ }^{2}$ ${ }^{1}$ Ege University School of Dentistry, Izmir / Bornova, Turkey, ${ }^{2}$ izmir Katip Çelebi University School of Dentistry, Izmir / Cigli, Turkey

Purpose: This study was aimed to observe the effect of different storage mediums on dentin moisture and microhardness in addition to the bond strength of a resin composite to dentin.

Methods: 30 human non-carious pre-molars were used. After extraction, each 10 teeth group was kept in \% 0.1 tyhmol, distilled water or dry storage for 7 days at room temperature. After sectioning of all teeth in longitudinally, enamel of the samples was removed. Vestibular parts of the samples were used for dentin moisture which were determined by grain moisture meter(M recieter 480,Japan). Following the dentin microhardness, microshear bond strength were measured on lingual dentin parts of the samples. The flowable resin composite cylinders(SDR, Dentsply,USA). in $0.9 \mathrm{~mm}$ diameter and $3 \mathrm{~mm}$ high were bonded to dentin using a self-etching adhesive(Clearfil SE Bond,Japan). Universal testing machine was used bond strength measurements.

Results: Moisture measurements of the dentin samples which were preserved various storage mediums presented significant differences $(\mathrm{p}<$ 0.05 ). Similarly, storage mediums affected bonding strenght of the resin composite to dentin $(\mathrm{p}<0.05)$. The highest dentin bond strength were obtained when distilled water was used for storage solution $(\mathrm{p}<0.05)$. However, microhardness measurements were not affected from different storage conditions $(\mathrm{p}>0.05)$. 
Conclusions: Within the limitations of this study, it was concluded that different storage mediums affected the dentin moistures and microshear bond strength test results except for microhardness.

Keywords: Dentin, Moisture, Storage medium, Micro-hardness, Microshear bond strength

\section{BM09}

Randomised crossover-controlled trial investigating poly-etherether-ketone (PEEK) materials in tooth-and-mucosa supported RPDs - Preliminary report.

Zaid Ali ${ }^{1,2}$, Nicolas Martin ${ }^{1,2}$, Sarah Baker ${ }^{1}$

${ }^{7}$ University of Sheffield, Sheffield, UK, ${ }^{2}$ Charles Clifford Dental Hospital, Sheffield, UK

Introduction: Poly-aryl-ether-ketone (PAEK) materials are marketed as non-metal alternatives for prosthodontic frameworks increasing the range of materials to consider when prescribing fixed and removable restorations. Despite the advantages of bone-mimicking modulus of elasticity, low weight to strength ratio, and biocompatibility from years of use as an orthopaedic device material, the introduction into the dental market has been a relatively recent development. Current evidence regarding the clinical use of these materials is limited to case reports and case series. The aim of this EPSRC funded randomised crossover-controlled clinical trial is to explore the differences between the use of poly-ether-etherketone (PEEK) framework removable partial dentures (RPDs) and traditional cobalt chromium $(\mathrm{CoCr})$ framework RPDs on oral health-related quality of life (OHRQoL), health-related quality of life (HRQoL) and gingival health.

Methods: In this pilot study twelve patients with a minimum of four missing teeth per dental arch (excluding third molars) have been provided with either CoCr or PEEK framework RPDs to wear for four weeks. Outcome measures in this pilot study are impact on OHRQoL measured by OHIP-20, HRQoL measured by the SF-12v2.0 and clinical outcomes including plaque index, bleeding on probing, tooth mobility and periodontal pocketing. After the first four weeks patients will be given the other denture to wear for a second four week period before outcome measures are repeated.

Preliminary Results: We present the results for the first twelve participants to be recruited to this pilot study.

\section{BM10}

Comparative study of the photo-polymerization depth of flow composite materials using Raman microscopy

Sebastiana Arroyo Bote, Javier Martinez Osorio, Esther Berastegui Jimeno

$\overline{\text { Faculty }}$ of Dentistry. Barcelona University, Barcelona, Spain

Objective: The aim of this study is to evaluate the depth of photopolymerization of different composite materials whose indications are Cavitary base flow in comparation with conventional flow hybrid composites.

\section{Materials and Methods:}

We have selected four Cavitary base flow materials (A) and four conventional flow hybrid composites (B).

\section{GROUP A:}

1. SDR TM (Dentsply)

2. Xtra base (VOCO)

3. Vertiseflow (Kerr)

4. everX Posterior + IFU (GC)

GROUP B:

6. X-Flow (Dentsply)

7. Grandioso flow (VOCO)

8. Premise flowable (Kerr)

9. G-aenial universal Flow (GC)

For photo-polymerization have been selected Demi Plus LED Dental Curing Light $1100 \mathrm{~mW} / \mathrm{cm} 2$ (KERR).

We have prepared ten samples for each material, in cylinders of 3,5mm of diameter and $10 \mathrm{~mm}$ of height. Ever cylinder was polymerized during 10 seconds. The polymerized cylinders have been studied by microscopy Raman analyzing the spectra of the material along the height of the cylinders, realizing measurements every 100 microns.

Results: The results demonstrate the differences between the eight materials. The statistical study with no parametric probe (Kruskal-Wallis), revealed significant differences between the results obtained from the two groups (A-B), the best results were from the group A. We also found significant differences in the depth of the polymerization between the four materials that we analyzed in group A,(U of Mann-Whitney). The most successful results were EverX Posterior.

Conclusion: The polymerization carried out with the high intensity lamp for 10 seconds, was lower for Cavitary base flow group in comparation with conventional flow hybrid composite.

\section{BM11}

\section{Fatigue Testing of Ultrathin Occlusal Veneers}

Karl-Heinz Kunzelmann ${ }^{1}$, Peter Rösch ${ }^{2}$, Helena Schäfer ${ }^{1}$ ${ }^{T}$ LMU-München, Operative Dentistry, Munich, Germany, ${ }^{2}$ Univ. Applied Sciences, Augsburg, Computer Science, Augsburg, Germany

Introduction: Occlusal veneers $(\mathrm{OV})$ are a conservative alternative during the treatment of occlusal erosions in order to reestablish the original occlusal height and protect the tooth from further occusal tissue loss. While traditionally a ceramic thickness of $1.5 \mathrm{~mm}$ is recommended by the manufactures for occlusally loaded ceramic restoration material improvements make a reevaluation of this recommendation necessary.

Materials and Methods: The teeth were anatomically reduced so that dentin was exposed on the whole occlusal surface and ultrathin $\mathrm{OV}$ were manufactured with defined thickness using a CEREC CAD/CAM machine. The thickness of the OV was 0.5- 
$0.8 \mathrm{~mm} .20$ samples of each material (Vivadent: IPSEmpressCAD, IPSe.maxCad, 3M/ESPE: LavaUltimate) were adhesively luted with Variolink II after pretreatment of the tooth surface with Syntac Classic (total-etch). The restorative materials were pretreated according to manufacturer's recommendations. The teeth were fatigue loaded in a fatigue simulator for $10^{\wedge} 6$ cycles with $50 \mathrm{~N} /$ cycle. Every decade the damage was investigated. As soon as a crack was visible with lateral illumination the sample was considered to be a loss.

Results: The cumulative survival probability (Kaplan-Meier) was: Empress 0.5 ( 9 losses after $10^{\wedge} 4$ cycles, 1 after $10^{\wedge} 5$ cycles), E.max 1.0, LavaUltimate 0.95 ( 1 loss after $10^{\wedge} 5$ cycles). The $95 \%$ CI suggests a significant difference between Empress and E.max/ LavaUltimate, while no difference was obvious between E.max and LavaUltimate.

Conclusions: Based on the high-cycle fatigue simulation and the rigorous failure criterion the modern materials E.max and LavaUltimate should a feasible alternative for the treatment of occlusal erosions with ultrathin occlusal veneers.

\section{BM12}

\section{Finite Element Analysis of Ultrathin Occlusal Veneers}

Peter Rösch $^{1}$, Karl-Heinz Kunzelmann ${ }^{2}$

${ }^{T}$ Augsburg University of Applied Sciences, Faculty of Computer Science, Augsburg, Germany, ${ }^{2}$ Ludwigs-Maximilians University, Munich, Dept. of Restorative Dentistry and Periodontology, Munich, Germany

Introduction: In order to systematically develop ultrathin occlusal veneers, an experimental fatique simulation has been complemented with a finite element analysis (FEA) based on micro CT images of the very same specimen. A quantitative comparison of different materials and geometries requires configurable software allowing for semi automatic volumetric analysis and visualisation.

Materials and Methods: Extracted teeth with ultrathin occlusal veneers were imaged using a Scanco Micro-CT 40 device. The 3D images (isotropic resolution: $0.04 \mathrm{~mm}$ ) were segmented with ImageJ and itk-SNAP. Orchestrated by a configurable python script, tetrahedral meshing and linear FEA were performed using iso2mesh and z88 with Young's moduli of 95.0, 84.1, 18.6 and 5.0 GPa for ceramic, enamel, dentine and glue respectively. Mimicking experimental conditions, a distributed surfcace load of $100 \mathrm{~N}$ was applied to restored cusps. For a comparison with resin nano ceramic materials, FEA was repeated with a modulus of $13.0 \mathrm{GPa}$ for the restoration. Results were visualised and statistically analysed with ParaView and numpy.

Results: Plausibility of results could be verified by visual inspection. Statistical analysis of volume weighted maximum principle stress values within the restoration based on about 200000 tetrahedra yields the following percentiles: Median: 0.30 (0.066) GPa, $\mathrm{P}(0.9): 2.0$ (0.57) GPa, P(0.99): 5.8 (4.3) GPa and $\mathrm{P}(0.999): 8.7$ (5.5) $\mathrm{GPa}$ where stress values in brackets correspond to nano ceramic material.

Conclusions: Based on free software, a tool chain for FEA has been established and successfully applied to ultrathin occlusal veneers. The study will be extended to a larger number of samples.

\section{BM13}

Bonding of a CPP-ACP containing glass ionomer cement to human enamel, sound-, and caries-affected dentine

Michael Burrow ${ }^{1}$, Hiroyasu Koizumi ${ }^{2}$, Hamdi Hamama ${ }^{3}$

${ }^{T}$ University of Melbourne, Melbourne, Australia, ${ }^{2}$ Nihon University, Tokyo, Japan, ${ }^{3}$ Mansoura University, Mansoura, Egypt

Glass ionomer cements are regarded as one of the few materials that reliably bond to enamel and dentine as well as assist with inhibition of dental caries. To aid caries inhibition and remineralisation of early lesions, casein phosphopeptide amorphous calcium phosphate (CPP-ACP) has been incorporated into Fuji VII (Fuji Triage). Previous laboratory work has shown the addition of CPP-ACP reduces enamel demineralisation.

The purpose of this study was to evaluate the microtensile bond strength of a conventional and the CPP-ACP containing glass-ionomer cement (GICs) to human enamel, sound-, and caries-affected dentine (CAD).

Twenty non-carious teeth and 10 teeth exhibiting frank dentine caries lesions (human molars) were used. A conventional GIC (Fuji VII, GC Corporation, Japan) or CPP-ACP-modified GIC (Fuji VII EP, GC Corp) were used as the test materials. Microtensile bond strengths were measured and analyzed statistically using two-way ANOVA at $\alpha=0.05$. The morphology of bonded interfaces were observed with SEM.

The results of the two-way ANOVA showed that the bond strength was not significantly affected by the type of 'GICs' $(p=0.183,>0.05)$ and 'teeth substrates' $(p=0.390,>0.05)$. Furthermore, the interaction between 'GICs' and 'teeth substrates' was also not significant ( $p=0.349,>0.05)$. Mean bond strengths of the Fuji VII was 3.8 MPa for enamel, 5.3 MPa for sound-dentine, and 5.0 MPa for CAD, and Fuji VII EP was 5.4 MPa for enamel, 5.4 MPa for sound-dentine, and 5.1 MPa for CAD. The results demonstrated that the adhesion of CPP-ACP containing GIC was no different to that of the conventional GIC.

\section{BM14}

\section{Effect of a cavity cleanser/desensitizing agent on bond strengths}

Hiroyasu Koizumi $^{1,4}$, Michael Burrow ${ }^{2}$, Hamdi Hamama ${ }^{3}$ ${ }^{T}$ Nihon University School of Dentistry, Tokyo, Japan, ${ }^{2}$ University of Melbourne, Melbourne, Australia, ${ }^{3}$ Mansoura University, Mansoura, Egypt,

${ }^{4}$ The University of Hong Kong, Hong Kong, Hong Kong

Recently a silver diamine fluoride and potassium iodide solution (Riva Star) has been introduced for clinical use as a desensitizing agent or biofilm inhibitor. Little is known whether bond strengths are affected by application of this material.

The aim of this study was to determine whether Riva Star influenced bond strengths to an etch and rinse (Optibond FL), 2-step self-etching (Clearfil Liner Bond F) and all-in-one (Optibond Versa) resin-based adhesive and a resin-modified GIC adhesive (Riva Bond LC).

Human mid-coronal dentine was the substrate used. The adhesives were bonded according to the manufacturers' instructions or after the dentine surfaces had been treated with the Riva Star. Teeth were sectioned into $1 \mathrm{~mm} \mathrm{X}$ $1 \mathrm{~mm}$ samples and subjected to a microtensile bond strength test. Results were analysed using univariate analysis of variance and Sidak's multiple test. 
It was shown bond strengths were adversely affected for all adhesives. Control: Optibond FL 32.1 MPa, Clearfil Liner Bond F 28.4 MPa, Optibond Versa 35.0, Riva Bond LC 18.4 MPa; Riva Star treated groups: Optibond FL 21.4 MPa, Clearfil Liner Bond F 10.8 MPa, Optibond Versa 9.6 MPa, Riva Bond LC 14.5 MPa.

Only the GIC and etch and rinse adhesive were less effected by the Riva Star application. SEM analysis showed that Riva Star left a precipitate on the dentine surface which adversely affects self-etching systems.

It was concluded that Riva Star should not be recommended to cleanse cavities but used only for spot application where a cavity is close to the pulp.

\section{BM15}

Conversion Degree and Micro-Hardness two dual cements cured with three different protocols under Zirconia discs

Stefano Serra, Allegra Comba, Luca Fontanive, Milena Cadenaro, Elio Berutti, Nicola Scotti

University of Turin, Department of Surgical Sciences, Turin, Italy

Objectives: The aim of this in vitro study was to assess the conversion degree (DC) and the micro-hardness (MH) of two dual cements employed under zirconia discs and cured with three different time protocols. The null hypothesis is that different curing protocols are not able to improve $\mathrm{DC}$ and $\mathrm{MH}$ of the cement.

Methods: 30 Zirconia discs of $2 \mathrm{~cm}$ of diameter were prepared. Discs were divided into two groups $(\mathrm{n}=15)$ according to the cement employed: 1) Rely-X (3M, ESPE); 2) Panavia V5 (Kuraray). Each group was further divided into 3 subgroups $(\mathrm{n}=5)$ : a) no light; b) $20 \mathrm{sec}$ cure; c) $120 \mathrm{sec}$ cure. Polymerization was performed $60 \mathrm{sec}$ after the sample was placed on the diamond support of a FT-IR spectrophotometer of new generation (Thermo Scientific Nicolet IS10) with a high power multiLED lamp (Valo-Ultradent). Final DC\% were calculated after 10 minutes. Once the conversion degree has been established, a Vickers Test with a micro-indentometer was performed. ANOVA test was performed to find differences between $\mathrm{DC} \%$ and $\mathrm{HM}$ of the two cements.

Results: One-way Anova showed that the two cements reached higher $\mathrm{DC} \%$ in subgroup $\mathrm{b})$ and $\mathrm{c})(\mathrm{p}<0.05)$. Further, Rely-X showed higher $\mathrm{DC} \%$ than Panavia2.0 only after 20 sec cuing $(\mathrm{p}<0.05)$. As concern $\mathrm{MH}$, Anova test showed that an increase in curing time is able to improve $\mathrm{MH}$ significantly both for Rely-X and Panavia2.0.

Conclusions: The null hypothesis has to be rejected since DC\% and $\mathrm{MH}$ of the dual cements tested were influenced by the curing time protocol.

\section{BM16}

\section{Color changes of expired restorative composite resins}

Nikolina Spyropoulou, Anastasia Plevritaki, Panagiotis Lagouvardos Dental school of Athens, National Kapodistrian university of Athens, Athens, Greece

Purpose: Composite resins are not to be used after their expiration date but a number of clinicians do make restorations with such materials. Since information on the stability in staining solutions of these materials is lacking, the purpose of this study was to evaluate the changes of expired composites within coffee as a staining solution.

Materials and Methods: 3 composite resins were selected from a pool of expired(X) after a significant time period, but kept in standard conditions(ice/SDI-15months,rok/SDI-89months, Spectrum/Dentsply-146months).Non expired(N) composites from the same materials were also selected and discoid specimens $(2 \mathrm{~mm}$ in thickness by $6.5 \mathrm{~mm}$ in diameter) were made from all materials. All specimens were finished with $\mathrm{SiC}$ wetordry paper up to 1000 grit, polished on a velvet microcloth and stored initially for $48 \mathrm{~h}$ in water of $37 \mathrm{oC}$ and then in coffee baths for 2 hours, 4 hours and 6hours, simulating a 30days,60days and 90days staining effect of coffee (two daily drinks of coffee- $4 \mathrm{~min} /$ day). A mean of three measurements of color parameters in the CIELAB system were taken in all periods with a portable contact colorimeter(ShadeEye/Shiofu) and the data were analyzed statistically by 2 wayANOVA at $\mathrm{a}=0.05$.

Results: Color difference values $\left(\Delta \mathrm{E}^{*}\right)$ between base line and the staining periods for the investigated materials were 4.9,9.3,10 for rokX,5.2,11.9,13.8 for rokN,10.8,19.7,29.6 for spectrumX,17.2, 31.4,38.8 for spectrum and 8.7,27.9,23.7 for iceX,11.3,23.9,20.2 for iceN. 2way ANOVA indicated no differences between expired and not expired materials but significant differences between staining periods.

Conclusions: Although investigated materials had expired for long, their staining behavior within coffee were not different from not expired materials.

\section{BM17}

$\mu$ TBS of an etch-and-rinse adhesive system enriched with Chlorhexidine-digluconate Loaded Nanodroplets

Stefania Rivarossa ${ }^{1}$, Diletta Basile ${ }^{1}$, Riccardo Tempesta ${ }^{1}$, Marta Gai $^{2}$, Caterina Guiot $^{3}$, Roberta Cavalli ${ }^{3}$, Elio Berutti ${ }^{1}$, Nicola Scotti ${ }^{1}$

${ }^{I}$ Dental School, University of Turin, Torino, Italy, ${ }^{2}$ Department of Molecular Biotechnology and Health Sciences, University of Torino, Torino, Italy, ${ }^{3}$ Department of Neurosciences, University of Turin, Torino, Italy

Purpose: Drug Loaded Nanodroplets (DLNs) could be employed for MMPs Inhibition Agents delivery and release in dentin. The aim of this in vitro study was to evaluate the influence of dentin pre-treatment with Chlorhexidine-digluconate loaded Nanodroplet (CHXLNs) on the bond strength of an etch-andrinse adhesive system.

Methods and Materials: 10 molars crowns were flattened and standardized smear layer was created. Exposed dentin surfaces were etched with $36 \%$ phophoric acid for $15 \mathrm{sec}$. Sample were divided in two groups according to the application (G1) or not (G2) of CHXLNs for $30 \mathrm{sec}$ and dried. Then, in all samples, Schotchbond Universal was applied and cured for $60 \mathrm{sec}$ at $1400 \mathrm{~mW}$ with Valo. Then resin composite was applied. Specimens were serially sectioned to obtain 1-mm-thick beams in accordance with the $\mu$ TBS test technique. Beams were stressed to failure after 7 days of storage in artificial buffer. Two more samples were prepared and sectioned in order to observe hybrid layer with confocal laser scanning microscopy. One-way Anova was performed to evaluate the effects of CHXLNs pre-treatment on the bond strength of and etch-and-rinse adhesive system. 
Results: Mean bond strength in G1 was $35.03 \pm 8.94 \mathrm{MPa}$, in G2 was $38.73 \pm 8.97$. ANOVA test showed any influence of CHXLNs pretreatment on the bond strength of adhesive tested. CLMS showed that CHXLNs are present under adhesive resin tags.

Conclusion: Within the limits of this study we can conclude that CHXLNs do not influence bond strength of etch-and-rinse adhesive system. The effect of CHXLNs on bond strength durability is under investigation.

\section{BM18}

Influence of Synthetic Hydroxyapatite application on fiber posts bond strength and durability.

Diletta Basile $^{1}$, Emanuele Bergantin ${ }^{1}$, Gianluca Turco ${ }^{2}$, Milena Cadenaro $^{2}$, Lorenzo Breschi ${ }^{3}$, Elio Berutti ${ }^{1}$, Nicola Scotti ${ }^{1}$

${ }^{1}$ Dental school, university of Turin, Torino, Italy, ${ }^{2}$ University of Trieste, Department of Medical Sciences, Trieste, Italy, ${ }^{3}$ Alma Mater Studiorum, University of Bologna, DIBINEM., Bologna, Italy

Objective: The aim of this in vitro study was evaluate the effect of Calcium-Phosphate minerals application on fiber post bond strength and durability.

Methods: 64 single-root teeth were endodontically treated and a $10 \mathrm{~mm}$ post space was prepared in each sample. Specimens were randomly assigned to four groups $(\mathrm{n}=16)$ according to the fiber post luting procedures. G1: EDTA10\% + self-adhesive cement (Panavia SA,Kuraray); G2: EDTA $10 \%$ + TeethMate Desensitiser (Kuraray) + self-adhesive cement (Panavia SA,Kuraray) G3: AllBond Universal (Bisco) + Core-X Flow (Dentsply); G4: AllBond Universal (Bisco) + TeethMate Desensitiser (Kuraray) + Core-X Flow (Dentsply). Teeth were cut in $1 \mathrm{~mm}$ thick slices; sample were pushed until failure with an Instron Machine after 24h and 12 months of storage in artificial buffer at $37^{\circ} \mathrm{C}$. 2 samples per group were prepared for the SEM analysis. An EDS (EDAX Integrated GENESIS APEX 2i EDS System with Apollo X Silicon Drift Detector) detector was used together with the SEM for elemental analysis of the sample's surface. Results were statistically analyzed with one-way ANOVA test $(\mathrm{p}<0.05)$.

Results: Statistical analysis showed a significant difference in bond strength between G1 and G2 (p=0,0001) and between G3 and G4 (p= 0,001 ). Hydroxyapatite application significantly helps to prevent bond strength decrease overtime $(p=0.0001)$. EDAX analysis showed that TeethMate created a Calcium-Phosphate precipitation over dentinal tubules.

Conclusion: Within the limitations of an in vitro study we can affirm that the creation of calcium-phosphate precipitation over dentinal walls could improve and stabilize bond strength of fiber post luted with 10 MDP-containing adhesive systems.

\section{BM19}

\section{Ultrashort pulsed lasers - Effects on dental tissues and restorative} materials

Joerg Meister $^{1}$, Florian Schelle ${ }^{2}$, Claudia Dehn ${ }^{1}$, Matthias Frentzen ${ }^{1}$ ${ }^{T}$ Dept. of Periodontology, Conservative and Preventive Dentistry, Bonn University, Dental Faculty, Bonn, Germany, ${ }^{2}$ Institute for Occupational Safety and Health of the German Social Accident Insurance (IFA), Sankt Augustin, Germany
In recent years ultrashort pulsed lasers (USPL) were introduced into dental laser research. The aim of this technology is to ablate oral hard and soft tissues as well as restorative materials with minimal collateral damages at sufficient ablation rates under the condition of high precision and minimal pain.

Basic investigations with the present technology were carried out with $\mathrm{Nd}: \mathrm{YVO}_{4}$ laser systems emitting light in the spectral range of $1 \mu \mathrm{m}$ at pulse durations of $8 \mathrm{ps}$ and pulse repetition rates of $500 \mathrm{kHz}$. The average power is $9 \mathrm{~W}$. With a $\mathrm{M}^{2}$ of 1.1 and a beam diameter of $30 \mu \mathrm{m}$, the corresponding intensity of the laser is $300 \mathrm{GW} / \mathrm{cm}^{2}$. Additionally, comparable high power ablation rate measurements were performed employing a laser source with an average power up to $50 \mathrm{~W}$. Irradiations were carried out with a scanner system and without a water spray.

Threshold values in the range of $0.45 \mathrm{~J} / \mathrm{cm}^{2}$ (composite) to $1.54 \mathrm{~J} / \mathrm{cm}^{2}$ (enamel) were observed. An ablation rate of up to $37.53 \mathrm{~mm}^{3} / \mathrm{min}$ for composite material was achieved with the $9 \mathrm{~W}$ laser source. Be employing the $50 \mathrm{~W}$ laser, increased rates up to $\sim 50 \mathrm{~mm}^{3} / \mathrm{min}$ i.e., for dentin were obtained.

The current state of this technology regarding dental material processing (oral and restorative materials) including advantages, limitations and future prospects of this technology will be presented.

\section{BM20}

Antimicrobial Activity of Octenidine Dihydrochloride, Polyhexanide and Chlorhexidine Digluconate Against Cariogenic Microorganisms

Esra Uzer Celok ${ }^{1}$, Mustafa Ates ${ }^{2}$, Ayse Tugce Tunac ${ }^{1}$, Bilge Hakan Sen ${ }^{3}$ ${ }^{1}$ Department of Restorative Dentistry, Faculty of Dentistry, Izmir Katip Celebi University, Izmir, Turkey, ${ }^{2}$ Division of Basic and Industrial Microbiology, Department of Biology, Faculty of Science, Ege University, Izmir, Turkey, ${ }^{3}$ Private Practice, Izmir, Turkey

Aim: The aim of this study was to assess the antimicrobial in-vitro effects of octenidine dihydrochloride, polyhexanide and chlorhexidine digluconate on cariogenic microorganisms by using their minimum inhibitory concentrations (MIC) and minimum bactericidal concentrations (MBC).

Materials and Methods: Streptococcus mutans (ATCC 25175), Lactobacillus acidophilus (ATCC 4356), Lactobacillus rhamnosus (ATCC 7469) and Actinomyces viscosus (ATCC 15987) were submitted to broth microdilution of octenidine dihydrochloride and polyhexanide at $1000-3 \mathrm{mg} / \mathrm{L}$ and chlorhexidine digluconate at $10.000-30 \mathrm{mg} / \mathrm{L}$. After the tubes were incubated at $37^{\circ} \mathrm{C}$ for $48 \mathrm{~h}$ and they were read in a spectrophotometer in order to detect the MIC. A new subculture was placed on agar plates to obtain the $\mathrm{MBC}$.

Results: Different MIC and MBC were observed in all disinfectants against each microorganism. The lowest $\mathrm{MIC}$ and $\mathrm{MBC}$ against $\mathrm{S}$. mutans $(60 \mathrm{mg} / \mathrm{L})$ were obtained from polyhexanide while the lowest values against L. acidophilus ( $15 \mathrm{mg} / \mathrm{L}, 30 \mathrm{mg} / \mathrm{L}) \mathrm{L}$. rhamnosus $(15 \mathrm{mg} / \mathrm{L}, 30 \mathrm{mg} / \mathrm{L})$ and $\mathrm{A}$. viscosus $(30 \mathrm{mg} / \mathrm{L})$ were determined by octenidine dihydrochloride.

Conclusion: Octenidine dihydrochloride and polyhexanide have the potential to be replaced with chlorhexidine digluconate as they were effective on cariogenic microorganisms. 


\section{BM21}

\section{Microleakage of Class II cavities restored with bulk-fill restorative resins}

Tugce Guney, A.Ruya Yazici

Hacettepe University, School of Dentistry, Department of Restorative Dentistry, Ankara, Turkey

Objective: The aim of this in vitro study was to evaluate the microleakage of Class II cavities restored with different restorative resins; bulk-fill resin composite, bulk-fill flowable resin, fiber-reinforced resin composite and a nanohybrid resin composite.

\begin{abstract}
Materials and Methods: Forty-eight extracted non-caries human molars were used for the study. In each tooth standardized Class II (slot) cavities were prepared (on mesial and distal surfaces) with one having the gingival margin below, one above the cemetoenamel junction (CEJ). After preparation the teeth were randomly assigned into four groups of twelve specimens each $(n=12)$. The cavities were restored with; Group 1: Tetric EvoCeram Bulk Fill (Ivoclar/Vivadent); Group 2: SureFil SDR Flow + Ceram X Mono (Dentsply); Group 3: GCeverX posterior + G-aenial posterior(GC Corp.); Group 4: Filtek Supreme(3M ESPE). Each restorative material was used with its' respective adhesive system. The restored teeth were stored in distilled water for $24 \mathrm{~h}$ at $37{ }^{\circ} \mathrm{C}$ and then thermocycled $\left(5-55^{\circ} \mathrm{C}, \mathrm{x} 1000\right)$ before immersion in $0.5 \%$ basic fuchsin dye for $24 \mathrm{~h}$. The teeth were then rinsed and sectioned mesiodistally and dye penetration was scored under a stereomicroscope(20X). Data were statistically analyzed using the Kruskal-Wallis and Mann-Whitney U tests $(p<0.05)$.
\end{abstract}

Results: The Kruskal-Wallis test revealed no significant differences in microleakage scores among all groups either on enamel $(p=0.296)$ or on dentin $(p=0.788)$.

Conclusion: None of the restorative materials tested completely eliminated microleakage. Nanohybrid resin composite, fiber-reinforced resin composite and bulk-fill resins either flowable or not showed similar microleakage values.

\section{BM22}

Effect of exposure distance with MultiLED lamps on surface microhardness in a bulk-fill composite.

Oana Andreea Miclaus, Allegra Comba, Damiano Pasqualini, Mario Alovisi, Elio Berutti, Nicola Scotti

University of Turin, Department of Surgical Sciences, Dental School, Turin, Italy

Objectives: The aim of this in vitro study was to assess the effect of exposure distance on surface microhardness obtained with different MultiLED curing lights of a bulk-fill composites. The hypothesis is that polywave multiLED curing lights induce uniform microhardness distribution on composite surface independently of curing distance.

Methods: A bulk-fill composite (Filtek Bulk Fill Posterior, 3M ESPE) was selected and placed in a $2 \mathrm{~mm}$ metallic mould.
Samples were exposed to irradiation with 3 different curing lights: TwoWave-Heraeus (Group1),Valo-Ultradent (Group 2), Swiss Master Light-EMS (Group 3) at the same energy density (27J/ $\mathrm{cm} 2$ ). Polymerization was performed with the curing tip at a distance of $0 \mathrm{~mm}, 2 \mathrm{~mm}, 4 \mathrm{~mm}, 6 \mathrm{~mm}$ and $8 \mathrm{~mm}$ from the top surface of the sample. All specimens were stored in distilled water in a light-proof container at $37^{\circ} \mathrm{C}$ for 24 hours. Top and bottom surface of samples were submitted to Vickers test on the central and peripheral area. Analysis of variance (ANOVA) was performed to evaluate the effects of light distance, sample zone (central vs peripheral), curing light and their interactions on microhardness. All statistical analyses were performed at a significance level of 0.05 .

Results: Exposure distance significantly influenced microhardness with all tested lamps $(\mathrm{p}=0.0001)$. Between curing units, TwoWave performed significantly better than Valo and Halogen light $(\mathrm{p}=0.0001)$. Sample central area was significantly harder than peripheral area in all groups $(p=0.0001)$.

Conclusions: The initial hypothesis is rejected, since exposure distance, curing light and surface zone all significantly affected surface microhardness of bulk-fill resin composite.

\section{BM23}

Cytotoxicity of high temperature pressure polymerized resin infiltrated ceramic networks on human oral stem cells

Eric Bonte ${ }^{1,4}$, Mathilde Tassin ${ }^{2}$, Tsouria Berbar ${ }^{2}$, Ludwig Loison Robert ${ }^{1}$ ${ }^{2}$, Michael Sadoun ${ }^{1,3}$, Benjamin Fournier ${ }^{5,2}$

${ }^{I}$ Paris Descartes University, Sorbonne Paris Cité, Dental faculty, Paris, France, ${ }^{2}$ INSERM UMR 1138, Cordeliers research center, Paris, France, ${ }^{3}$ URB2i, Paris Descartes Dental faculty, Paris, France, ${ }^{4}$ Bretonneau Hospital, AP-HP, Paris, France, ${ }^{5}$ Paris Diderot University, dental faculty Garancière, Paris, France

Background: The development of CAD - CAM techniques revealed the need for new materials suited to this technique and offering a safe and sustainable clinical implementation. The infiltration of resin in a ceramic network under high pressure and high temperature defines a new class of hybrid materials for this purpose which requires to be evaluated by cytotoxicity. We used oral stem cells (gingival and pulpal) as an in vitro experiment model.

Methods: In this study, 4 biomaterials were powdered and assessed at $2 \mathrm{mg} / \mathrm{ml}$ : Enamic (Vita), Experimental hybrid material (EHM), EHM with initiator (EHMi) and polymerized Z100 composite material $(3 \mathrm{M})$ on oral stem cells. Viability, apoptosis, proliferation, cytoskeleton, inflammatory response, oxidative stress and morphology (SEM) were evaluated. Statistical analysis (ANOVA) was performed using GraphPad Prism6 (Graphpad Software inc, 6.0) software.

Results: Proliferation was delayed by all the tested biomaterials but no difference was observed between them. Study of the cytotoxicity revealed less viable cells in presence of Z100 (MTS assay), however no significant apoptosis occurred. Cytoskeleton and extracellular matrix synthesis were not altered and an intimate contact appeared between materials and cells.

Conclusion: The four polymerized and powdered materials tested did not exhibit adverse effects on oral stem cells in our experimental conditions. 


\section{BM24}

Which methodological factors influence the shear-bond strength of fissure sealants?

Vinay Pitchika ${ }^{1}$, Meng-Ling Chiang ${ }^{2}$, Sebastian Birlbauer ${ }^{1}$, Alexander Crispin $^{1}$, Reinhard Hickel ${ }^{1}$, Nicoleta Ilie ${ }^{1}$, Jan Kühnisch ${ }^{1}$

${ }^{1}$ Ludwig Maximilians University of Munich, Munich, Germany, ${ }^{2}$ School of Dentistry, Taipei, Taiwan

Objective: This study aimed to investigate the influence of different methodological variables on shear-bond strength (SBS), e.g. tooth surface, enamel pretreatment, specimen alteration and shearing technique.

Methods: 180 healthy, caries-free, extracted human third molars were selected. All roots were sectioned off $1 \mathrm{~mm}$ apical to cement-enameljunction and the crowns were divided into four surfaces (mesial/ distal/ oral/ buccal). The resulting 720 tooth pieces were embedded, numbered and randomly allocated to the pre-treatment (prismatic/ aprismatic enamel and 30/60sec etching time), alteration (1day/3months water storage/ 5000x thermocycling), and shearing method (Ultradent/ Guilliotine). Finally, 18 groups each containing 40 specimens ( 10 of every surface) were formed. All sealants (Helioseal, Vivadent, Schaan, Liechtenstein) were placed using the cylindrical mould insert which was fixed in a bonding clamp (ISO 29022) according to the specification of each group. After specimen storage, the shearing force was measured in a universal testing machine (MCE 2000ST, Quicktest, Langenfeld, Germany). Unpaired ttests, one-way ANOVA, linear regression and multiple linear regressions were used for data analysis.

Results: There were significances between the groups under t-test and one-way ANOVA test. All of the included factors were significant under linear regression, which were then included into multiple linear regression; resulting in a significant model with aprismatic enamel, distal surface and 5000x thermocycling as significant factors.

Conclusion: While specimen preparation and thermocycling showed an significant influence on SBS, the shearing method and chosen enamel surface might act as confounders and should be controlled in future invitro sealant trials.

\section{BM25}

Immediate adhesive properties to dentine of two "universal" adhesives

Maria Cura, Ana Hurtado, Maria Victoria Fuentes, Laura Ceballos $\overline{R e y ~ J u a n ~ C a r l o s ~ U n i v e r s i t y, ~ M a d r i d, ~ S p a i n ~}$

Objective: To determine microtensile bond strength ( $\mu \mathrm{TBS}$ ) to dentine and degree of conversion inside the hybrid layer (DC) of two "universal" adhesives using self-etch or etch-and-rinse strategies in comparison with a 2-step self-etch adhesive.

Methods: 50 extracted caries-free human molars were assigned to five groups: 1) the "universal" adhesive Xeno Select (Dentsply), applied as a one-step self-etch adhesive (XE-1SE); 2) Xeno Select, applied as a 2-step etch and rinse adhesive (XS-2ER); 3) the "universal" adhesive Scotchbond Universal adhesive (3M ESPE), applied as a one-step selfetch adhesive (SBU-1SE); 4) Scotchbond Universal adhesive applied as a 2-step etch-and-rinse adhesive (SBU-2ER); 5) A 2-step self-etch adhesive, Clearfil SE Bond (CSE, Kuraray). Specimens were prepared for $\mu \mathrm{TBS}$ test and for DC determination by micro-Raman spectroscopy. Data were analyzed by ANOVA and Tukey test $(\mathrm{p}<0.05)$.

Results: $\mu$ TBS mean values in MPa (standard deviation) were: XE-1SE $18,24(12,8)^{\mathrm{b}}$; XS-2ER 22,71 $(12,0)^{\mathrm{b}}$; SBU-1SE 55,94 $(23,6)^{\mathrm{a}}$; SBU2ER 59,30 (16,4) ${ }^{\text {a }}$; CSE 58,77 $(23,3)^{\text {a }}$; and DC in \% (standard deviation) were: XE-1SE 53,34 (16,5) ${ }^{\text {b}}$; XS-2ER 70,19 $(11,2)^{\text {a }}$; SBU-1SE 76,32 $(13,7)^{\mathrm{a}}$; SBU-2ER 77,25 $(16,5)^{\mathrm{a}}$; $\operatorname{CSE} 79,16(10,8)^{\mathrm{a}}$.

Conclusions: Clearfil SE and Scotchbond Universal, used as 2-step etchand-rinse or as a one-step self-etch adhesive, yielded higher bond strength to dentin than Xeno Select. Xeno Select as a self-etch adhesive had a significantly lower DC than the other testing groups.

\section{BM26}

Bisphenol A release: a comprehensive survey on the composite resin composition.

Elisabeth Dursun ${ }^{1,3}$, Jean-Pierre Attal ${ }^{1,4}$, Hélène Fron ${ }^{1,4}$, Anne Raskin ${ }^{1,5}$ ${ }^{T}$ Paris Descartes University, Paris, France, ${ }^{2}$ Aix-Marseille University, Marseille, France, ${ }^{3}$ A. Chevenevier Hospital, Creteil, France, ${ }^{4}$ C. Foix Hospital, Ivry-sur-Seine, France, ${ }^{5}$ La Timone Hospital, Marseille, France

Introduction: Bisphenol A (BPA) is an endocrine disruptor with a potential toxicity. If the composite resins (CR) do not contain pure BPA, its derivatives are widely used. Several studies reported BPA levels and its derivatives in the saliva or urine after the placement of CR. The aims of our study were: to establish an exhaustive list of the CR and their composition; to assess to what extent BPA derivatives are used.

Method: A search on the manufacturers' websites was performed to reference all the $\mathrm{CR}$ marketed in Europe. A comprehensive search of their composition was then carried out from both material safety data sheets and standardized questionnaire sent to manufacturers. They should: indicate if their CR contained the monomers listed; add other monomers; note " no communicated ", if they did not want to give any details. The results were recorded and analyzed using the Excel software.

Results: 160 CR have been identified in 31 manufacturers. 24 manufacturers $(77,4 \%)$ responded to our survey. When they have not responded $(22,6 \%)$, the web research was not sufficient to complete the CR composition. $61 \%$ are based on bis-GMA, or bis-GMA and UDMA; $15 \%$ on UDMA; $5 \%$ do not contain bis-GMA, nor UDMA; for 19\%, it was not communicated. One CR contains bis-DMA.

Conclusion: Even the scientific evidence encouraging to stop using CR with BPA derivatives, it may seem logical to select CR without them, for the population at risk (children and pregnant women). This work has established a list of 19 possible products.

\section{BM27}

Vickers Micro-Hardness evaluation of two fiber-post dual cements cured with three different time protocols

Maria Luisa Favale, Elena Manzon, Dorina Selaj, Allegra Comba, Elio Berutti, Nicola Scotti 
University of Turin, Department of Surgical Sciences, Dental School, Torino, Italy

Objectives: The aim of this in vitro study was to assess the effect of three different curing protocols on the micro-hardness $(\mathrm{MH})$ of one selfadhesive and one dual-curing cement. The null hypothesis tested is that different waiting times before light curing do not influence $\mathrm{MH}$ of dualcuring cements.

Methods: To conduct the in vitro study, 48 cement samples were prepared using a custom-made metallic mold that simulates post-space in terms of depth, diameter and light conditions. Samples were divided into 2 groups ( $\mathrm{n}=24)$ according to the dual-curing cement employed: 1$)$ Selfadhesive resin cement (Rely-X Unicem 2, 3M ESPE); 2) Dual-curing cement (Clearfil DC Core, Kuraray). Each group was further divided into three subgroups considering the curing protocol: a) $60 \mathrm{sec}$. curing after one minute of waiting time; b) $60 \mathrm{sec}$. curing after five minutes of waiting time; c) $60 \mathrm{sec}$. curing after nine minutes of waiting time. Vikers MH for each sample, for both the coronal and the apical area, was obtained using a nano-indentometer. One-way Anova was performed to evaluate the effects of different waiting times before polymerization on $\mathrm{MH}$ of the coronal and apical portion.

Results: Anova test showed that for the three subgroups of both cement there was a significantly higher $\mathrm{MH}$ in the coronal portion $(\mathrm{p}=0.0001)$. Further, significant $\mathrm{MH}$ differences $(\mathrm{p}=0.0001)$ concerning curing protocol were found only for DC Core.

Conclusions: The null hypothesis is partially not rejected because longer waiting time before curing only improve $\mathrm{MH}$ values of DC Core and not of Rely-X Unicem2.

\section{BM28}

Effect of etch-and-dry and etch-and-rinse application of universal adhesives on post-operative sensitivity: randomized clinical trial

Gabriele Petrini, Marco Zante, Oana Miclaus, Nicola Scotti, Damiano Pasqualini, Mario Alovisi, Elio Berutti

Dental School, University of Turin, Turin, Italy

Purpose: In this in vivo study we evaluated the influence of application technique of three universal on immediate post-operative sensitivity (POS).

Methods: In total, 100 patients with one posterior tooth affected by a primary carious lesion were recruited. The patients were randomly divided into three groups, based on the universal adhesive systems employed: group A (All Bond Universal, Bisco); group B (Scotchbond Universal, $3 \mathrm{M} \mathrm{ESPE}$ ); group C (Futurabond U, VoCo). Every adhesive was randomly applied with etch-and-rinse (application of 36\% phosphoric acid for 15 $\mathrm{sec}$ ) or etch-and-dry technique. The same skilled operator carried out the restorations using a nano-hybrid composite (GrandioSo, Voco) in oblique stratification. Each increment was light-cured for $20 \mathrm{~s}$ with a LED lamp. Patients received a three-level thermal sensitivity form for post-operative sensitivity ratings. The evaluations were recorded daily for 7 days. POS trends were analyzed with the Friedman test $(\mathrm{p}<0.05)$.

Results: All (100\%) enrolled patients completed the follow-up visits. There was no statistically significant difference between the three groups in post-operative sensitivity trends. In every groups, increased postoperative sensitivity was found at $\mathrm{T} 1$, which then decreased significantly during the observation period. Adhesive application technique did not significantly influence POS.

Conclusion: The results of this clinical trial showed that universal adhesives, applied either with etch-and-rinse or etch-and-dry technique, avoided POS after direct posterior composite restorations. Follow-up visits of treated patients are scheduled to analyze clinical longevity of restorations performed in this clinical trial.

\section{BM29}

Mercury in Dental Amalgam. What do North Iraqi Patients think?

Abdülkadir Sengun ${ }^{1}$, Hakan Colak ${ }^{2}$, Errtugrul Ercan ${ }^{1}$, Mehmet Mustafa Hamidi $^{1}$, Hassan Al Yousuf ${ }^{0}$

${ }^{1}$ Kirikkale University School of Dentistry Department of Restorative Dentistry, Kirikkale, Turkey, ${ }^{2}$ Ishik University School of Dentistry Department of Restorative Dentistry, Erbil, Iraq

Aim: The aim of this study is to assess the awareness about the toxicity of mercury in dental amalgam among sample of dental patients in North of Iraq.

Material methods: A cross-sectional survey was conducted among 518 dental outpatients who attended Ishik University School of Dentistry Clinics.Data were collected using structured questionnaires. They were asked ingredients of the amalgam, opinion about mercury pollution in the environment, health problems associated with mercury.

Results: Thirty four percent of respondents claimed that silver fillings contain mercury. The majority of respondents stated that they will prefer higher cost fillings contain no mercury rather than lower cost fillings that contains mercury. There is overwhelming opinion $(96,3 \%)$ that dentists should be required to inform their patients about the various types of mercury and non-mercury fillings available before filling a cavity. Approximately $21,8 \%$ reported mercury may cause health problems in Human Beings while 73 , $5 \%$ claimed they don't have any idea. Only $3.6 \%$ of participants claimed to receive dental amalgam despite prior knowledge of its mercury content.

Conclusion: This study demonstrated that general knowledge and awareness of toxicity of mercury in dental amalgam is relatively low among North Iraqi dental patients

\section{BM30}

Effect of polywave multiLED curing lights on surface microhardness distribution of two nanofilled composites.

Marco Zante, Allegra Comba, Damiano Pasqualini, Mario Alovisi, Elio Berutti, Nicola Scotti

University of Turin, Department of Surgical Sciences, Dental School, Turin, Italy

Objectives: The aim of this in vitro study was to assess the effect of different poliwave MultiLED curing lights on surface microhardness 
topography of two nanofilled composites. The hypothesis is that polywave multiLED curing lights induce uniform microhardness distribution on composite surface.

Methods: A non-carious molar tooth, extracted for periodontal reasons, was selected. Crown was horizontally sectioned 2-mm above the CEJ. A $3 \times 4-\mathrm{mm}$ Class I cavity was prepared in order to obtain a "tooth mould". Composite samples, $2 \mathrm{~mm}$ thick, were prepared using the tooth mould and divided in two groups $(\mathrm{n}=$ 10 each) according to the composite (GrandioSo - VoCo, Germany; Filtek XTE - 3M ESPE, USA). Samples were cured with 5 curing lights (TwoWave-Heraeus, Valo-Ultradent, Bluephase G2Ivoclar Vivadent, Demi Ultra-Kerr, Swiss Master Light-EMS) at the same energy density $(27 \mathrm{~J} / \mathrm{cm} 2)$. After 7 days of storage, composite samples were submitted to Vickers test on the central and peripheral area. Analysis of variance (ANOVA) was performed to evaluate the effects of curing light and specimen zone (central vs peripheral) and their interactions on microhardness.

Results: Two-way Anova showed comparable results for both composites (Filtek and GrandioSo). The two variables (lamp and zone) statistically affected micro-hardness $(\mathrm{p}<0.0001)$, but not the interactions between them.

Conclusions: The two composites employed showed a decrease in micro-hardness values from the central to the peripheral area for all five curing lamps, thus the initial null hypothesis must be rejected. Probably the hardness distribution of composite surface reflects the irradiance distribution of each curing light tested in this study.

\section{BM31}

Effect of the interface geometry on the stress distribution of the restored tooth-ceramic crown complex

Raad Al Marza, Shirin Shahrbaf, Nicolas Martin The University of Sheffield, Sheffield, UK

Objective: The effect of the cement interface geometry on the structural integrity of the tooth ceramic-crown complex is poorly understood. This work investigates, by means of three-dimensional finite element analysis (3D FEA) the effect of the interface geometry(thickness and uniformity)on the stress state of the tooth ceramic-crown complex.

Method: Two typodont maxillary first premolar teeth were restored with lithium disilicate all-ceramic crowns fabricated to create two different interface geometries: (i) Wide and non-uniform (mean $157 \mu \mathrm{m} \pm 36$ ) and; (ii) thin and uniform (mean $114 \mu \mathrm{m} \pm 19$ ). These were adhesively cemented using Variolink II resin cement (Ivoclar-Vivadent AG, Liechtenstein) and subsequently digitized with a micro-CT scanner. An image processing software program (Mimics, Materialise Ltd)and haptic technology (Freeform, SensAbleTechnologies Inc) were used to generate a 3D model and supporting structures (PDL, alveolar/cortical bone). These high-resolution 3D digital models of the restored tooth and supporting structures were converted into 3D-FEA models using the Hypermesh software package v.10.0 (Altair Engineering Inc). The boundary condition was defined at the outermost surface of the supporting bone. Each of the structures was assumed to be homogenous, with an isotropic structure and have linear elastic behaviour. A static occlusal load of $300 \mathrm{~N}$ was applied and the resultant generated stresses were calculated (von Mises and Principal).
Result: The stress values were less in the ceramic crown and cement structures of the thinner and more uniform interface space model.

Conclusion: The stress distribution in the tooth components varied greatly between models and was affected by the cement interface thickness and uniformity.

\section{BM32}

Degree of conversion and micro-hardness of a light-curing cement under lithium-disilicate and composite discs

Atdhe Grezda, Allegra Comba, Damiano Pasqualini, Mario Alovisi, Elio Berutti, Nicola Scotti

University of Turin, Turin, Italy

Objective: evaluate the conversion degree (DC) and the micro-hardness $(\mathrm{MH})$ of light curing cement under lithium disilicate and composite discs of different thicknesses. The null hypothesis were that (1) thickness and (2) material do not influenced resin cement DC and MH.

Methods: 15 lithium-disilicate discs (E-Max CAD) $2 \mathrm{~cm}$ of diameter, A2 shade LT and 15 composite discs (LavaUltimate 3M), A2 shade LT, were prepared and divided into three groups $(n=5)$ according to the thickness: A) $0.6 \mathrm{~mm}$; B) $1 \mathrm{~mm}$; C) $1.5 \mathrm{~mm}$. Adhesive procedures were performed on a single surface of each disc following manufacturer instruction and Choice2 light-curing cement (Bisco) was applied. Curing was performed with a multiLED polywave lamp (Valo) for $60 \mathrm{sec}$ at $1700 \mathrm{~mW} / \mathrm{cm} 2$ with the lamp in slight contact with the opposite surface towards the cement layer. DC\% of the cement was evaluated with ATR FT-IR spectrophotometer (Thermo Scientific Nicolet IS10) both for the ceramic and the composite discs. Once the DC was established, a Vickers Test was performed on the cement with a micro-indentometer. Three indentations were performed on each sample and the mean value was considered for statistical analysis. ANOVA test was performed to evaluate the influence of thickness and material on $\mathrm{DC} \%$ and $\mathrm{MH}$.

Results: ANOVA showed that DC\% were not dependent on material and thickness while $\mathrm{MH}$ values were affected by both thickness and material $(\mathrm{p}<0.05)$.

Conclusion: Null hypothesis (1) and (2) were not rejected since only MH values were influenced by thickness and material when a light curing cement is used for cementation.

\section{BM33}

Quality of Final Impressions and Prescriptions for Fixed Restorations.

Neamat Abubakr ${ }^{1,2}$, Riham Nabil ${ }^{1}$

${ }^{7}$ University of Khartoum, Faculty of Dentistry, Khartoum, Sudan, ${ }^{2-}$ Princess Nourah Bint Abdelrahman University, College of Dentistry, Riyadh, Saudi Arabia

Purpose: The purpose of this study was to investigate the quality of final impressions and prescriptions for fixed restorations that were received by private and educational institute's laboratories. 
Methods: Two hundred and seventeen questionnaires were distributed to eight private laboratories and seven dental schools. The questionnaires were filled by the dental technicians; who were trained and instructed to complete one questionnaire per one final impression for fixed restoration immediately after it was received.

Results: Total response rate was $87 \%$; the responding technicians had found evidence of obvious contamination in $23.8 \%$ of the examined impressions; blood was clear in $68.9 \%$ of these cases. Metal stock trays were used in $57.1 \%$ of the examined sample. About two-third of the impressions had been taken using condensation silicone (64.6\%). Poor or no written instructions were observed in half of the sample $(n=103)$.

Conclusions: Within the limitation of the present study, results showed an inappropriate selection of material for final impression; the crossinfection control of the sent impressions was below standard and there were poor prescriptions and communications with laboratory technicians. Clinical Implications: Proper infection control need to be applied for both dentists and dental technician. Appropriate impression material and proper communication with dental laboratory technicians will definitely improve the quality of fixed prosthodontics work.

\section{BM34}

Surface rougness of nano-hybrid composites before and after immersing in sports drink

\author{
Elif Ozturk $^{1}$, Gizem Guder ${ }^{2}$ \\ ${ }^{T}$ Hacettepe University, Ankara, Turkey, ${ }^{2}$ Kocaeli University, Kocaeli, \\ Turkey \\ Objectives: The purpose of this study was to evaluate the differences in \\ surface roughnesses of two different nano-hybrid composite after im- \\ mersing in a sports drink.
}

Methods: Fourty disk-shaped specimens (diameter:10-mm;thickness:2$\mathrm{mm}$ ) were prepared from two different nano-hybrid resin composites (Cavex Quadrant Universal LC, Cavex, Haarlem, The Netherlands and Tetric N-Ceram, Ivoclar Vivadent, Schaan, Liechtenstein). Each specimen was cured under the mylar strips for 20 seconds from each of top and bottom surfaces. All the specimens were stored in distilled water for $24 \mathrm{~h}$ at $37^{\circ} \mathrm{C}$. Baseline measurements of surface roughness (Ra) values were performed with a 3D-optical-profilometer (NewView ${ }^{\mathrm{TM}} 7200$, Zygo). Specimens were immersed in a sports drink (Redbull, The CocaCola Co., Turkey) 3 minutes daily for a period of 30 days. Control groups were stored in distilled water only. Surface roughnesses were measured after the immersion period. For each composite 3 measurements were performed and the average was recorded. Data were statistically analyzed using Univariate Analysis of Variance test (SPSS 20.0) $(\mathrm{p}=0.05)$.

Results: There was statistically no difference between the Ra values of the study groups before or after immersing in distilled water or sports drink $(\mathrm{p}>0.05)$

Conclusions: The tested sports drink did not cause any alteration in 3Dsurface roughnesses of the selected nano-hybrid composites for a 30-day evaluation period.

\section{BM35}

Effect of water storage and brushing on dentinal permeability of teeth treated with desensitizing agents

Luca Debernardi ${ }^{1}$, Emanuele Bergantin ${ }^{1}$, Gianluca Turco ${ }^{2}$, Elio Berutti ${ }^{1}$, Nicola Scotti ${ }^{1}$
${ }^{1}$ University of Turin, Department of Surgical Sciences, Dental School, Torino, Italy, ${ }^{2}$ University of Trieste, Department of Medical Sciences, Trieste, Italy

Objectives: The aim of this in vitro study was to assess the effect of ageing and electric brushing on dentinal permeability of samples previously treated with three different desensitizing agents. The null hypothesis is that dentinal permeability is not influenced by ageing and periodical electric brushing.

Methods: 18 intact molars were selected, occlusal enamel and crowns were then cut in $0,5 \mathrm{~mm}$ slices, perpendicular to the long axis of the teeth, in order to obtain $1 \mathrm{~mm}$ thick dentin discs. Samples were than randomly divided into 3 groups according to desensitizing treatment performed: 1) ReminPro (Voco); 2) AllBond Universal (Bisco); 3) TeethMate Desensitizer (Kuraray). Groups were further divided into 2 subgroups considering different ageing treatment: a) storage in artificial saliva; b) subgroup a) treatment +10 min standardized brushing once a week for 3 months. Dentin permeability was assessed before and immediately after treatment, after 3 months of ageing treatment. Samples were subsequently prepared for SEM analysis to evaluate differences in tubules occlusion. Kruskal-Wallis and Wilcoxon test were performed to evaluate dentin permeability changes before and after samples treatment $(\mathrm{p}<0.05)$.

Results: Statistical analysis showed that water storage $(p<0.001)$ and brushing $(\mathrm{p}<0.0001)$ significantly increased dentin permeability except for TeethMate Desensitizer.

Conclusions: All occluding agents reveal comparable tubular occlusion at baseline SEM analysis. However, statistical analysis showed that water storage and daily brushing increase dentin permeability for all groups except for TeethMate Desensitizer that remain stable both after storage in artificial saliva and controlled brushing.

\section{BM36}

Effect of immediate or delayed dentinal sealing on coronal bond strength: $\mu$ TBS test

Francesco Lerda University of Turin, Turin, Italy

Objective: The aim of this in-vitro study was to evaluate the influence of immediate or delayed adhesive system application on coronal dentin bond-strength. The null hypothesis is that immediate dentinal sealing (IDS) do not provide higher bond strength than delayed dentinal sealing (DDS), independently of the adhesive technique.

Methods: 30 molars crowns were flattened and standardized smear layer was created with 600 grit paper. Samples were randomly divided in 6 groups according to adhesive treatment:

G1 IDS with 3step etch-and-rinse (Optibond FL, Kerr)

G2 IDS with EDTA10\% for 1min, TeethMate Desensitizer (Kuraray), water rinse for 10sec, 2step self-etch adhesive application (Clearfil SE Bond 2, Kuraray).

G3 IDS with 2step self-etch adhesive application (Clearfil SE Bond 2).

G4 DDS (after 8weeks of storage in artificial saliva at $37^{\circ} \mathrm{C}$ ) with G1 procedure 
G5 DDS with G2 procedure

G6 DDS with G3 procedure

Then $4 \mathrm{~mm}$ resin composite was applied and cured with LED lamp. Specimens were serially sectioned to obtain 1-mm-thick beams in accordance with the $\mu$ TBS test technique. Beams were stressed to failure after 24h. Two-way Anova was performed to evaluate the effects of immediate/delayed sealing and adhesive procedure on coronal bond strength.

Results: Two-way Anova showed that adhesive technique did not influenced immediate bond strength, while delayed bond strength was significantly worst $(\mathrm{p}=0.0001)$.

Conclusion: Null hypothesis are partially refused since the immediate dentinal sealing provide higher bond strength, independently of the adhesive technique.

\section{BM37}

\section{Effect of Er-YAG or bur on bond strength with different adhesive procedures}

aristea cedrone, allegra comba, riccardo tempesta, elio berutti, nicola scotti University of Turin, Turin, Italy

Purpose: Er-YAG laser treatment could influence adhesive effectiveness. The aim of this in vitro study is to evaluate the bond strength obtained with different adhesive strategies on coronal dentin cutted with Er-YAG laser or bur

Methods and Materials: 40 molars crowns were flattened and standardized smear layer was created. Samples were divided in 2 groups according to the dentin cutting technique $(\mathrm{n}=20)$ : Er-YAG laser or bur. Every group was then dividend in subgroups $(n=5$ each) according to the adhesive technique:

SG1 dentin etching for $15 \mathrm{sec}$ and universal adhesive application (AllBond Universal, Bisco)

SG2 universal adhesive application (AllBond Universal)

SG3 2step self-etch adhesive application (Clearfil SEBond 2, Kuraray)

SG4 3step etch-and-rinse adhesive application (Optibond FL, Kerr)

Adhesives were all cured for $60 \mathrm{sec}$ at $1400 \mathrm{~mW}$ with Valo. Then resin composite was applied. Specimens were serially sectioned to obtain 1-mm-thick beams in accordance with the $\mu$ TBS test technique. Beams were stressed to failure after 7 days of storage in artificial buffer. Two-way ANOVA was performed to evaluate the effects of cutting technique (CT) and adhesive technique (AT) and their interaction on the coronal dentin bond strength. Significance was set at $\mathrm{p}<0.05$.

Results: AT showed significant influence on coronal dentin bond strength $(\mathrm{p}=0.0001)$, while CT did not.

Conclusion: Within the limits of this study we can conclude that Er-YAG treatment on coronal dentin did not affect the bond strength, above all with etch-and-dry techniques. Further investigations on bond strength stability are necessary.
BM38

Pulp chamber temperature increase during adhesive system irradiation with multiLED curing lights.

Giulia Massano, Francesca Barra, Francesco Coero Borga, Damiano Pasqualini, Elio Berutti, Nicola Scotti

University of Turin, Turin, Italy

Objective: The aim of this in vitro study was to compare temperature rise in the pulp chamber induced by different lights when curing adhesive system. The null hypothesis is that curing lights (1) and irradiation time (2) do not influence temperature increase.

Methods: 60 intact molars were selected. A first class cavity $3 \mathrm{~mm}$ depth with pulpal floor $1 \mathrm{~mm}$ distant from the pulp chamber was prepared on each sample. Roots were cut, a J-Type thermocouple was inserted into the pulp chamber that was then sealed. Samples were divided in 4 groups $(n=$ 15) according to the curing light employed: Swiss Master Light (EMS), TwoWave (Heraeus), Valo (Ultradent), Bluephase Style (Ivoclar). Adhesive system (Clearfill Universal, Kuraray) was applied and irradiated with different time regimens (10sec, 20sec, and 40sec). Temperature rise in the pulp chamber, time to reach maximal temperature (tMax) and to return to initial temperature (tBack) were registered. Results were statistically analyzed with ANOVA test $(\mathrm{p}<0.05)$.

Results: There were no statistically significant differences between halogen and multiLED tested curing lights regarding pulp chamber temperature increases when polymerizing a universal adhesive, independently of the irradiation time. The greatest temperature increases were observed with Valo (2.1 degrees Celsius), followed by halogen (1.4 degrees Celsius) and TwoWave and Bluephase Style (0.9 degrees Celsius). tBack was significantly higher $(\mathrm{p}=0.0001)$ with halogen light.

Conclusion: Null hypothesis was accepted since curing lights tested did not differ in temperature increase induction, independently of irradiation time. However, halogen light induce more persistent heat in the pulp chamber.

\section{BM39}

Mulitomodal Optical Characterization of dentine caries lesions: multiphoton imaging, spectroscopy and micro-hardness analysis

Shara Sajini, TIim Watson, Frederic Festy

King's Colleage London, London, UK

Carious dentine is described clinically and histologically as two altered zones, a superficial infected and a deeper affected layer. Based on important structural features, the delineation between these layers is essential, both clinically and in laboratory investigations. The aim of this study was to evaluate and correlate objectively the autofluorescence (AF) features of sound, infected and affected carious dentine with their microhardness characteristics using a multimodal approach

A total of 20 extracted carious molars were used in the study, a reference mark was created in each half. Using both halves: fluorescence and fluorescence life-time imaging (FLIM) in a two-photon microscope, localized areas were imaged, recorded, and the fluorescence spectrum was detected from each point as well as the second harmonic generation image (SHG). Afterwards, a single photon spectrometer was used to detect AF signal from the same areas. Finally, the Knoop microhardness (KHN) of these 
areas was recorded and allocated subjectively into the three dentine zones. Significant statistical differences were found between the three zones delineating infected, affected and sound areas using these optical techniques. In conclusion, multiphoton imaging and single photon spectroscopy are considered alternative non-invasive in-vitro diagnostic methods for caries characterisation through the detection of SHG signal, AF signal, and fluorescence lifetime without dye labelling. These techniques have the potential to improve in-vitro cavity preparation through the better discrimination of the caries excavation endpoint, so avoiding excessive removal of tooth structure. Consequently, they may also facilitate reliable evaluation of caries affected dentine remineralisation with bioactive materials.

\section{BM40}

\section{Are the biomimetic therapies promising for direct pulp capping?}

Elif Aybala Oktay ${ }^{1}$, Ülkü Özan $^{2}$, Fulya Toksoy Topçu ${ }^{1}$, Huseyin Tort ${ }^{1}$ ${ }^{T}$ Gulhane Military Medical Academy,Department of Restorative Dentistry and Endodontics, Etlik/Ankara, Turkey, ${ }^{2}$ Abant Izzet Baysal University, Bolu, Turkey

Objective: To examine the cytotoxic effects of Emdogain(EMD) a purified acidic biomaterial containing amelogenins which are obtained from pig in the embryological term and which are enamel matrix derivatives out of dentin bonding agents as pulp capping in the dental pulp therapies with RTCA-XCELLigence impedance method.

Materials-Methods: The cytotoxic effects of four different dentin agents(Tokuyama Bond Force, Shofu Beauti Bond, Dentsply Prime\&Bond NT, 3M Adper Single Bond) used as pulp capping material in the direct pulp therapies and Emdogain(EMD), a enamel matrix derivative, and a purified acidic biomaterial containing amelogenins obtained from pig in the embryological term on the dental pulp cells, with RTCA XCELLigence impedance method were analyzed.

Dental Pulp cell cultures were used between the third to sixth passage only in this study. Dental Pulp cell cultures were transferred to the wells of $x$ celligence to be used in the subsequent phases of this study. It was evaluated with real time cellular impedance method whether or not different doses of DBA's added in media caused effects upon dental pulp in vitro. 1/250, 1/500 and $1 / 1000$ dilutions of all medications were used.

Result: We found out that all doses of EMD decreased the cell growth based upon the days exposed and it showed no cytotoxic effect. Our results showed that some dentin bonding agents have cytotoxic effects on dental pulp cells and they may inhibit dental pulp regeneration by decreasing cell proliferation.

Conclusion: It can be concluded that Emdogain is a material which can be preferred for pulp capping against dentin bonding agents.

\section{BM41}

Characterization of resin ceramic as compared to polymer-infiltrated ceramic-network material and dense ceramics

Hazem Abouelleil ${ }^{1}$, Alexis Goujat ${ }^{2}$, Dominique Seux ${ }^{1,2}$, Julia Bosco ${ }^{3}$, Pierre Colon ${ }^{1,3}$, Brigitte Grosgogeat ${ }^{1,2}$, Nelly Pradelle ${ }^{1,3}$

${ }^{1}$ Laboratoire des Multimatériaux et Interfaces ; UMR CNRS 5615 , Université Lyon1, Lyon, France, ${ }^{2}$ UFR Odontologie, Université Lyon1 ; Service de Consultations et de Traitements Dentaires ; Hospices Civils de
Lyon, Lyon, France, ${ }^{3}$ UFR D'odontologie, Université Paris Diderot; APHP, Hôpital Rothschild, Service d'Odontologie, Paris, France

Objectives: Determine the fracture toughness, flexural strength and modulus, and Vickers Hardness of a Resin Ceramic CAD/CAM material, GC CERASMART $^{\mathrm{TM}}$, compared with commercially available materials: Lava $^{\mathrm{TM}}$ Ultimate, $3 \mathrm{M}$ (Resin Ceramic), VITA ENAMIC ${ }^{\circledR}$, VITA Zahnfabrik (polymer-infiltrated ceramic-network - PICN), VITABLOCS Mark II, VITA Zahnfabrik (feldspar ceramic) and e.max CAD, Ivoclar Vivadent (lithium disilicate glass-ceramic).

Methods: mini-bar specimens $(3.0 \times 3.0 \times 14 \mathrm{~mm})$ were prepared for each CAD-CAM material. The flexural strength and elastic modulus were measured using three-point-bending (ISO 6872:2008). Vickers microhardness measures (three measurements for each sample) were made with a microhardness tester (Struers, USA). The fracture toughness (KIc) of the materials tested was determined using the Single Edge V-Notch Beam (SEVNB) method in accordance with standard ISO 6872. Fracture surfaces were analyzed using stereomicroscope and scanning electron microscopy (SEM). Results were analyzed via ANOVA with Turkey's t-test $(\mathrm{p}>0.05)$.

Results: Significant differences were observed between the different CAD-CAD blocks. lithium-silicate glass ceramic performed better followed by the hybrid ceramic material and feldspar ceramic.

Conclusion: Results demonstrated that all the materials properties were within the acceptable range for fabrication of single restorations according to the ISO standard for ceramics (ISO 6872:2008). However, important differences between the materials tested are noticed as a function of their structure.

\section{BM42}

Compression Fracture Resistance of four different Glass-ionomer cements

Domagoj Glavina, Kristina Gorseta, Tomislav Skrinjaric School of Dental Medicine University of Zagreb, Zagreb, Croatia

Background: Aim of the study was to compare fracture resistance to compression of four different GIC materials filled in Class II type cavities.

Materials and Methods: For the study purposes, zirconia ceramic class II cavity models were prepared according to standard preparation. Each test group comprised 6 samples (models). In order to prepare restorations models were filled with four different materials: Equia Forte (GC), Equia (GC), Ketac molar (3MESPE) and Ionostar Molar (Voco). Materials were applied according to manufacturers' instructions and with aid of sectional (anatomical) matrix. Models were stored at $37^{\circ} \mathrm{C}(100 \%$ humidity $)$ for $24 \mathrm{~h}$ prior testing. Each specimen was tested using an Universal testing machine (LRX, Lloyd Instruments) at $1 \mathrm{~mm} / \mathrm{min}$ crosshead speed and loaded to fracture using compression rod (diameter $1,5 \mathrm{~mm}$ ), with $1 \mathrm{kN}$ load cell. Statistical analysis of data was performed by one-way analysis of variance (ANOVA) and Tukey HSD post hoc test.

Results: Equia Forte showed significantly better fracture resistance comparing to all other materials (Equia Forte 257,2N; Equia 222,1N; Ketac Molar 140,7N; Ionostar Molar 114,5N). Type of the fracture was cohesive 
in all cases. Most Equia cavity models (samples filled with Equia Forte and Equia) fractured together with the restorations (73\%).

Conclusion: Fracture resistance to compression of materials filled in Class II type ceramic cavities could be valuable testing procedure for GIC testing.

\section{Clinical Cases (CC)}

\section{CC01}

Full-mouth rehabilitation in a patient affected by severe attrition: interdisciplinary perio-conservative and esthetic approach

Vicente Faus-Matoses ${ }^{1}$, Ignacio Faus-Matoses ${ }^{2,1}$, Ana TeruelRodriguez $^{1}$, Vicente Faus-Llacer ${ }^{1}$

${ }^{T}$ University of Valencia. Master in Restorative Dentistry and Endodontics, Valencia/Valencia, Spain, ${ }^{2}$ University of Valencia. Master in Orthodontics, Valencia/Valencia, Spain

Objective: To illustrate a conservative approach combining surgical and restorative procedures to achieve the functional and aesthetic full-mouth rehabilitation of a patient with severe dental attrition, reduced vertical dimension and gummy smile.

Methods: Healthy 61 year-old female came to our dental clinic suffering from severe incisal wear and unsatisfied with her dental appearance. After clinical and radiographic examination a DSD was performed, from which we obtained a diagnostic wax-up. The wax-up was transferred to the mouth in order to carry out the clinical valuation of aesthetic parameters (anterior teeth's length: assessing the position of gingival zeniths and incisal edges) through photographs and videos and afterwards the treatment plan was performed which included:

- Gingivectomy in the region of upper incisives.

- Direct oclusal composites to increase the vertical dimension on the non-restored posterior mandibular teeth.

- Improvement of anterior teeth's aesthetics with minimally invasive feldspathic veneers.

- Replacement of all old obturations and prosthesis with resin, alumina and feldspathic restorations.x

- $\quad$ Replace the absent posterior teeth with implants.

Results: It was possible to obtain a successful functional and aesthetic full-mouth rehabilitation with conservative and minimally invasive techniques.

Conclusion: With an accurate diagnosis, treatment plan and the use of the suitable materials it is possible to achieve excellent aesthetics and functional level while preserving maximum tooth structure.

\section{$\mathrm{CCO2}$}

Zirconia restoration with CAD-CAM system after orthodontic forced eruption with fiber-reinforced-composite

Jun-Sung Park, Won-Gyun Chung, Yoon Lee

Yonsei University Wonju Severance Christian Hospital, Wonju, Gangwon-do, Republic of Korea
Introduction: If conventional restoration treatment of a tooth with advanced dental caries is not possible, orthodontic forced eruption can be one of the treatment options. For forced eruption in anterior teeth or premolars, the esthetic aspect as well as the functional aspect of the appliance has to be considered. In the past, metal wires and brackets caused unaesthetic appearance during forced eruption, and porcelainfused-to-metal crowns resulted in dark gingiva appearance underneath the margin. In this case, the tooth was restored with a zirconia crown using CAD-CAM system after orthodontic forced eruption with fiberreinforced-composite (FRC) and root canal treatment (RCT) of a premolar.

Case report: An 18 year-old male patient presented with advanced dental caries. After caries removal on the upper right second premolar, due to the deep subgingival margin, crown restoration after non-surgical RCT and orthodontic extrusion was planned. After discussing the esthetics with the patient, forced eruption using FRC and crown restoration with zirconia was recommended for the patient. After RCT, forced eruption of the tooth, and fiber post and core placement, the tooth was prepared for zirconia crown. Intraoral digital scan of the tooth was taken, and the tooth was restored with a zirconia crown fabricated by CAD-CAM system.

Conclusions: By using FRC, the esthetic as well as the functional needs of the patients can be fulfilled during the $2 \sim 3$ month treatment period. The crown restoration procedure may be more comfortable and esthetic by using intraoral digital scan, CADCAM system and zirconia crown.

\section{$\mathrm{CC03}$}

Interdisciplinary orthodontics-conservative treatment in a patient with overbite and attrition lesions

Vicente Faus-Matoses ${ }^{1}$, Ignacio Faus-Matoses ${ }^{2,1}$, Lorenzo ValeroRemohí $^{1}$, Vicente Faus-Llacer ${ }^{1}$

${ }^{T}$ University of Valencia. Master in Restorative Dentistry and Endodontics, Valencia, Spain, ${ }^{2}$ University of Valencia. Master in Restorative Dentistry and Endodontics, Valencia, Spain

Objective: to describe a conservative approach using minimally invasive techniques in order to restore aesthetic and solve hipersensitivity in a patient with overbite and dental attrition.

Methods: A 37 years old was referred to our department. Her chief complaint was the dissatisfaction with her smile and hypersensitivity. After a photo study and the clinical examination, an interdisciplinary treatment plan including orthodontics, surgical and conservative procedures was performed:

- Direct oclusal composites in posterior mandibular teeth were placed to increase vertical dimension and achieving enough anterior space for the veneers.

- Orthodontics, including bite turbos on anterior teeth to correct overbite by extrusion of posterior teeth.

- Once the orthodontic treatment ended, oclussion was corrected and anterior space was achieved, a diagnostic wax up of the upper and lower veneers was done, it was transferred to the patient's mouth for clinical evaluation in terms of shape, size, and length.

- Gingivectomy in the region of upper and lower anterior teeth was carried out. - Replacement of the old upper veneers and placement of prepless lower veneers, all the new restorations were made with feldspathic ceramics. 
Results: With this conservative approach we were able to obtain excellent aesthetic results solving hypersensitivity and functional issues with minimally invasive techniques.

Conclusion: With a conservative interdisciplinary treatment it is possible to obtain excellent aesthetic and functional results avoiding a great loss of tooth structure.

\section{$\mathrm{CCO4}$}

Interdisciplinary orthodontic conservative treatment in a patient affected by severe tetracycline stains, agenesias and malocclusion

Vicente Faus-Matoses ${ }^{1}$, Ignacio Faus-Matoses ${ }^{2,1}$, Elianys-Yoseli Mavares-Rodriguez ${ }^{1}$, Vicente Faus-Llacer ${ }^{1}$

${ }^{T}$ University of Valencia. Master in Restorative Dentistry and Endodontics, Valencia/Valencia, Spain, ${ }^{2}$ University of Valencia. Master in Orthodontics, Valencia/Valencia, Spain

Case study of a 42 year old male patient that came into the clinic requiring a very conservative aesthetic and functional improvement of his mouth, due to the extensive grinding in both dental arches caused by dental malocclusion and severe tetracycline stains.

Initially the treatment plan led to put direct occlusal composites in the posterior affected teeth for the pathological grinding in order to raise vertical dimension and guarantee steady anterior restorations.

Following the plan, the proper use of orthodontics stabilized the occlusion and also placed well dental units 13 and 23 in the position of upper lateral incisors due to the existing agenesia.

A DSD generated a suitable reference guide for the final restorative treatment, compound by first of all, a wax up and a mock up that was placed over the patients teeth to visualize the final result and to have a better guide for the conservative engraving procedure.

An addition silicone impression was made after engraving procedure and sent to laboratory confection of veneers.

In order to opaque tetracycline stains, an opaque mycrohibrid composite (Esthet -X Dentsply. Konstanz, Germany) was placed at the buccal surface of each teeth and on top of this; the final restoration was cemented (Calibra - Dentsply. Konstanz, Germany)

Correct diagnosis and the proposed treatment plan was effective to fulfill the restorative demands of both the dentist and patient in terms of a conservative restorative approach to the problem.

\section{$\mathrm{CCO5}$}

Interdisciplinary approach: orthodontics and minimal-prep feldspathic veneers to rebuild the anterior guidance

Vicente Faus-Matoses, Ignacio Faus-Matoses, Diana-Carolina RomeroMora, Vicente Faus-Llacer

University of Valencia, Valencia, Spain

Introduction: Orthodontic treatment provides the space and correct occlusion to choose the restorative material in order to minimize further wear. Interdisciplinary treatments with feldspathic veneers create a balance between morphology, function and aesthetics.

Method: Female patient, 34 years old, with attrition of the incisal edges of the maxillary central incisors. An orthodontic treatment was conducted to intrude incisors, to move apically the dentogingival complex and restore the missing coronal tooth structure improving the patient's smile esthetics and a harmonious occlusion. After the orthodontic treatment sequential photographs were taken and a DSD was carried out for esthetic and structural pre-evaluation. A mock-up guided by the DSD was placed in the patient's mouth to evaluate the esthetic parameters. A conservative treatment with Feldspathic veneers (Noritake EX -3, Kizaeco, Japan) was planned on teeth 11-21 to create an anterior guidance and bring central incisors and gums to appearance and function with acceptable parameters. Afterwards a slight gingivoplasty was completed to correct hyperplasia caused by the orthodontia. Teeth preparation was carried out through the mock-up in order to be less invasive as possible and then final cementation was with resin (Light Calibra Esthetic Resin Cement, Dentsply, Caulk).

Conclusion: Management of interdisciplinary treatment leads to the prognosis of recurrence associated with orthodontic movements. That makes it possible to restore function and aesthetics with a lasting and conservative approach. Feldspathic porcelain veneers provide esthetic value and are visually as close to natural teeth as possible.

\section{CC06}

Timing in an ortho-conservative-treatment in a patient with attrition due to overbite and decreased overjet

Vicente Faus-Matoses ${ }^{1}$, Igancio Faus-Matoses ${ }^{2,1}$, Carla García-Cuesta ${ }^{1}$, René-Domingo Botello-Torres ${ }^{1}$, Vicente Faus-Llácer ${ }^{1}$

${ }^{1}$ University of Valencia. Master in Restorative dentistry and Endodontics, Valencia, Valencia, Spain, ${ }^{2}$ University of Valencia. Master in Orthodontics, Valencia, Valencia, Spain

Introduction: Teeth wear can occur for different factors and even alter the patient's esthetics. A conservative management approach has been followed to preserve the esthetics in those patients using direct composites and the Dahl principle. Timeline in an interdisciplinary treatment is needed to obtain good results.

Description: A 27-years-old male came to the dental office complaining about sensitivity and discomfort in the anterior teeth. The clinical examination revealed dental wear involving palatal surface of anterior maxillary teeth and incisal edges of lower incisors as a result of a deep overbite and a decreased overjet.He also presented diastemas in the anterior maxillary teeth due to the small size of the lateral incisors. The treatment plan was:

First step: Increase the vertical dimension following the Dahl principle placing direct palatal composite veneers and creating a posterior open bite.

Second step: Subsequent extrusion of posterior teeth was achieved and aided with orthodontics. The incisal edges were maintained in the same position to preserve the patient's smile line and to avoid the creation of an inverted smile. After 15 months the overbite had decreased providing an adequate anterior occlusion. 
Third step: incisal edges of the lower incisors and the upper diastemas were restored with direct composite.

Conclusions: It is important to know the correct timing in cases of interdisciplinary treatments. Localized anterior worn dentition with an intact posterior dentition is possible to manage in a conservative way creating interocclusal posterior space following the Dahl principle aided by orthodontics and the use of direct composites.

\section{$\mathrm{CC07}$}

Conservative retreatment with biologically oriented preparation technique (b.o.p.t.), orthodontics and direct composites

Vicente Faus-Matoses ${ }^{1}$, Ignacio Faus-Matoses ${ }^{2,1}$, Ignacio VazquezNatividad $^{1}$, Vicente Faus-Llacer ${ }^{1}$

${ }^{T}$ University of Valencia. Master in Restorative Dentistry and Endodontics, Valencia, Valencia, Spain, ${ }^{2}$ University of Valencia. Master in Orthodontics., Valencia, Valencia, Spain

Objective: To describe a conservative approach using multiple restorative procedures including B.O.P.T. technique to re-establish function and periodontal health while aesthetics is improved.

Methods: A healthy 55 year-old female came to our dental office concerned about her periodontal health and oral aesthetics. The clinical examination showed:

- Inflamed gums in the anterior maxillary teeth due to the presence of splinted zirconia crowns with inadequate marginal fit from 3 to 3 , which presented cracked cores.

- Overbite and attrition lesions in posterior occlusal surfaces and anterior incisal edges of mandibular teeth with crooked incisors and reduced vertical dimension as consequence.

The treatment plan would include an interdisciplinary approach combining orthodontics and restorative procedures. Vertical dimension was increased with direct posterior composites. Inferior incisors were aligned with orthodontics and restored using also direct composite restorations. Conventional crowns (horizontally prepared) were replaced by alumina crowns using vertical preparations in order to preserve maximum tooth structure.

Results: The use of a B.O.P.T. technique achieved an optimal gingival condition avoiding an excessive loss of dental tissue. The restorative and minimally invasive treatment was able to solve the esthetic, inflammatory and functional issues successfully.

Conclusion: It is possible with a conservative approach and B.O.P.T. technique to achieve an excellent esthetic level while preserving gingival health and maximum tooth structure.

\section{$\mathrm{CC08}$}

\section{Assessment of Patients' Oral Health Related Quality of Life in Treat- ed Hypodontia Patients}

Motasum Abu-Awwad, Ken Hemmings, Saja Mannaa, Daljit Gill, Akil Gulamali

University College London - Eastman Dental Institute, London, UK Hypodontia has a negative impact on patients' oral health related quality of life (OHRQoL).
Aims 1: Assess OHRQoL of hypodontia patients after treatment at Eastman Dental Hospital (EDH)

2. Assess the associations between patients' reported OHRQoL with clinical dental assessment, patients' satisfaction with treatment provided and social factors 3. Compare the patients' OHRQoL following treatment with the British public norms.

Methods: Hypodontia patients who finished their dental treatment at EDH completed a questionnaire incorporating the16-item UK oral health related quality-of-life measure (OHRQoL-UK), a list of four questions about their satisfaction with treatment received and a comprehensive clinical assessment using modified USPHS criteria.

Results: The sample included 52 participants $(22$ males, 30 females; median age 28 years) who had completed treatment for at least 6 months (Range 6-60 months). Most participants reported high OHRQoL scores and satisfaction with treatment provided. Clinical assessment revealed 4 of the participants had complications requiring treatment. No association between the clinical assessment and participants' reported OHRQoL scores was found $(\mathrm{P}=0.15)$. Satisfaction with dental aesthetics and gender revealed a statistically significant association $(\mathrm{P}=0.006$ and $\mathrm{P}=$ 0.047 , respectively). The OHRQoL mean scores of the sample were statistically significantly higher than the OHRQoL mean scores of the British public norms for most age groups $(\mathrm{P}<0.05)$.

Conclusions: Satisfaction with dental aesthetics seemed related to high OHRQoL scores. Females tended to report higher OHRQoL scores than males. Hypodontia patients following treatment were likely to report higher OHRQoL mean scores compared to the British population.

\section{CC09}

Masking discolored enamel surface with opaquers and tints before direct composite veneering.

Orsalia Vjero, Andreas Spaveras, Maria Antoniadou, Maria Anagnostou Dental School, Athens, Greece

Introduction: Conservative and esthetic treatment of a single discolored anterior tooth is a great clinical challenge. This discoloration can be caused by various reasons, including intra-pulpal hemorrhage, dental trauma and endodontic treatment. If a bleaching procedure cannot be implemented or has been tried out without satisfactory outcome, other conservative procedures should be considered, such as a direct composite veneering. The opaque shades of composite resins still have inherent limitations in their opacification ability, especially when increments are very thin. Thus, in order to match the chromaticity of the adjacent teeth, the use of opaquers and tints is suggested. These materials can effectively mask dark enamel surface, after minimal preparation. Then different composite layers can be stratified, emulating the natural dentine and enamel tissues according to the layering technique.

Aim: The description of opaquers and tints' use in masking discolored enamel surface, prior to direct veneering with composite resins in representative clinical cases.

Case presentations: In the first case, discolored enamel surface of the left lateral incisor was covered with a microfill pink opaque (Pink Opaquer, Cosmedent). In a second one, the right central incisor was veneered after applying a nanohybrid tint (Inspiro, Edelweiss). In both cases application of opaque and tints over the prepared enamel surface was made after the polymerization of the bonding agent. Veneering was performed with a 
composite resin system (Filtek Supreme Ultra, 3M ESPE) with the layering technique.

Conclusion: Although opaquers can achieve satisfied aesthetic results their application is considered as a technically sensitive approach.

\section{CC10}

\section{Evaluating quality of life in different groups of patients}

Teresa Palomares-Muriana

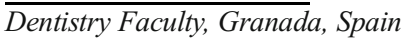

\section{Objective:}

This study was a controlled clinical trial aimed to assess how loss tooth affect oral health-related quality of life (OHRQoL) comparing two groups of patients: removable denture patients and non-removable denture patients classified by Eichner Index using the Oral Health Impact Profile (OHIP).

\section{Methods:}

The Oral Health Impact Profile OHIP-14 and OHIP-20 (for removable denture patients) was used to asses subjects' quality of life. Responses for each item are on a 5-point Likert scale $(0=$ "never" to $4=$ "very often"). All responses were summed to produce an overall OHIP score that could range from 0 to 56 (OHIP-14) or from 0 to 80 (OHIP-20), with high scores indicating poorer oral health-related quality of life.

Subjects were grouped into 3 groups by posterior occlusal contacts according to the Eichner Index.

\section{Results:}

The mean OHIP-14 score for group A was 8,86 (with 16 points highest score), for group B 20,62 (with 43 points highest score) and for group C was 17,50 (with 36 points highest score).

The average OHIP-20 score for group B was 17,67 (with 33 points highest score) and for group $\mathrm{C}$ was 50,22 (with 80 points highest score).

We observed higher scores therefore poorer oral health-related quality of life in C group- removable denture patients.

\section{Conclusions:}

Loss of occlusal contacts contributed to a poorer OHRQoL, even if the tooth/teeth were replaced by removable prostheses.

The use of conventional removable dentures brings negative impacts in the OHRQoL of patients which may have influenced the self-perceived discomfort with the prostheses.

\section{CC11}

Chairside-fabricated positioning stent for luting indirect restoration on a flat preparation: A case report

Joëlle Dulla, Adrian Lussi

University of Bern, Bern, Switzerland

Objective: The aim of this case report is to present a technique to simplify and quicken the luting of an indirect restoration on a flat preparation with precision and predictability.
Case Report: A 56-year-old male consulted for a cracked left maxillary first molar. Clinical and radiographic examination revealed that the tooth was necrotic. After endondontic treatment, a composite overlay was preferred because of the buccal-palatal fissure. This case report describes the use of a chairside-fabricated positioning stent for luting the restoration.

Discussion: Full cuspal coverage is advised when restoring pulpless teeth. Root posts are avoided whenever possible. The absence of a locked seating position for the indirect restoration on a flat preparation can render its luting difficult. The marginal fit of the restoration is important to its prognosis. When using dual resin cement, time is limited, but luting with filled composite can be challenging due to its consistency. In this report, the stent provided precise and predictable seating of the restoration as on the cast model. Removing the luting composite excess was easy. Minimal occlusal adjustment was needed. This technique was clearly advantageous in saving time and effort.

Conclusion: A positioning stent for the luting of an indirect restoration using filled composite was easily fabricated chairside on the cast model. The stent permitted fast, predictable and reproducible seating. Due to its design, luting composite excess was easily removed. The minimal occlusal adjustment required for the restoration was an indication of its precise seating. Stent design could be further improved.

\section{CC12}

48 months clinical performance of two current glass-ionomer systems in a field study.

Thomas Klinke ${ }^{1}$, Amro Daboul ${ }^{1}$, Roland Frankenberger ${ }^{2}$, Reinhard Hickel $^{3}$, Reiner Biffar ${ }^{1}$

${ }^{I}$ Greifswald University, Greifswald, Germany, ${ }^{2}$ Philipps-University Marburg und University Hospital Gießen and Marburg, Marburg, Germany, ${ }^{3}$ University Munich, Munich, Germany

Background: The aim of this clinical field study was to evaluate the clinical performance of EquiaFil ${ }^{\circledR}$ with a nano-filled resin coating (Equia) and conventional Fuji IX GP fast ${ }^{\circledR}$ with LC coating (GPfast) according to the FDI restoration material evaluation criteria.

Materials and Methods: Equia $(\mathrm{n} 1=515)$ and GPfast $(\mathrm{n} 2=486)$ were used to restore permanent teeth; class I (n1 = 146/n2 = 166), II mo/od $(\mathrm{n} 1=225 / \mathrm{n} 2=211)$ and class II $\bmod (\mathrm{n} 1=13$ / $\mathrm{n} 2=21$ ). Clinical examination, including photographs and stone casts, was carried out in a yearly-follow-up for 4 years by calibrated external examiners. Changes in aesthetic, functional and biological parameters (FDI_criteria: B5/B6/B8/B10/C11/C12/C13) over time were estimated with logistic mixed models adjusted for age, sex, fillings, patients and dentists.

Results: Both materials performed well in class I cavities, for class II mo/od, 16 fractures (GPfast) and 7 fractures (Equia) were observed. Odds ratios in adjusted random intercept models showed a protective treatment effect of Equia when placed within manufacturer's indication (odds ratio: 0.19 ) compared to GPfast. Filling size and surfaces was the most important component that affected clinical performance of both materials. Random intercept models adjusted for filling size showed approximatly two times higher odds for 2-surface fillings within 
and outside manufacturer's indication compared to fillings only within manufacturer's indications.

Conclusion: Both materials showed similar performance after 48 months in class I cavities. Within the manufacturer's indication, EquiaFil ${ }^{\circledR}$ showed a better overall performance with fewer failures in the followup intervals for class II (mo/od) fillings compared to GPfast. For better longevity, the manufacturers' recommendations have to be strictly followed.

\section{CC13}

Diagnosis and healing follow up of a cutaneous fistulous tract using 3D images.

Cristina González Losada ${ }^{1}$, Alfredo Saralegui Calvo ${ }^{1}$, Paula Saralegui González

${ }^{1}$ University Complutense, Madrid, Spain, ${ }^{2}$ University Complutense, Madrid, Spain, ${ }^{3}$ University Autónoma, Madrid, Spain

Introduction: The use of CBCT in endodontics should be limited to the assessment and treatment of complex endodontic conditions such us diagnosis of wide radiolucency lesions with no evidence of limits, identified with conventional imaging.

Clinical cases: We show clinical situations in which $\mathrm{CBCT}$ has been necessary to confirm our diagnosis. Conventional intraoral radiography provides clinicians with high-resolution imaging that continues to be the front-line method for dental imaging but there are situations in which due to its two dimensions images we can't get information like CBCT units, which reconstruct the data to provide interrelational images in three orthogonal planes (axial, sagittal and coronal).

The clinical case is a female, 30 years old who presents a cutaneous fistulous tract located next to the chin. This patient was in orthodontic treatment and we confirm the negative vitality of the right central inferior incisive. The vitality test was normal in all the other inferior teeth. We confirm the lesion and the limit extension and bone damage in the CBCT.

We show the endodontic treatment in this teeth and the control radiographies, as well as the evaluation of the recovery bone after one year and its confirmation with a new tridimensional radiographic technique ( CBCT).

Conclusions: $\mathrm{CBCT}$ has been definitive in this clinical situation to confirm lession extension limits and bone damage. It is necessary nowadays in Endodontics using CBCT as a complementary method of exploration and diagnosis. It allows confirmation of the pathology as well as evaluation of the recovery.

\section{CC14}

10-year follow up of a case of Amelogenesis Imperfect restored free hand with resin composites

L. Sebnem Turkun

Ege University School of Dentistry, Izmir, Turkey

Amelogenesis imperfecta (AI) is an inherited enamel dysplasia involving both dentitions with no other systemic effects. The hereditary pattern is autosomal or X-related dominant or recessive. Its prevalence is approximately 1:14,000-1:16,000. It can be classified as hypocalcified, hypoplastic and hypomaturated according to clinical, radiological, histological and hereditary findings.

This clinical report presents a case of hypomaturated type AI in a 16-yearold young man that was successfully treated with different types of resin composites and followed-up for 10-years. The patient's anterior teeth were restored with Filtek A110 (3M/ESPE, USA) and the posteriors with Filtek Z250 (3M/ESPE, USA) resin composite systems and with the help of Palodent matris system (Dentsply, USA). The finishing and polishing of the restorations were performed with diamond burs, Enhance \& PoGo polishing systems (Dentsply DeTrey, Germany).

The patient was recalled postoperatively every month for 6 months and then every 3 months for 2 years and then moved in another city for University education and did not attend his appointments. He came back 10 years later with a problem on his upper left canine. His oral hygiene was very bad, some marginal discolorations occurred on his anterior restorations but only 3 posterior restorations were lost. His impacted upper left canine teeth started to came in mouth. His oral hygiene was optimized, the posterior restorations refurbished/rebuild with a nanaohybrid resin composite G-aenial Posterior (GC, Japan) and the frontal teeth were re-restored again free hand with G-aenial (GC) a nanohybrid resin composite.

\section{CC15}

\section{Minimal intervention dental treatment for elderly patient}

Sevil Gurgan, Zeynep Bilge Kutuk, Filiz Yalcin Cakir

Hacettepe university, Ankara, Turkey

Purpose: Minimal intervention dentistry (MID) is a philosophy of care that aims to conserve tooth tissues throughout a persons' life with a modern medical approach to the management of caries, utilizing caries risk assessment, and focusing on the early prevention and interception of disease. The proportion of older adults in populations across the world is increasing, and this ageing population is going to present dentists with significant challenges. In this case presentation, minimally invasive restorative treatment of an elderly patient was demonstrated with four principals of MID.

Method: After clinical and radiographic examinations of a 76 year old male patient who was seeking oral care, potential caries risk factors were evaluated (1) by using saliva (Saliva-Check buffer, GC), bacteria (Saliva-Check Mutans Test, GC) and plaque analysis (Plaque Indicator kit, GC). Oral self-care, consist of toothbrushing twice daily with fluoride toothpaste was recommended. In addition to oral hygiene procedures, floride (MI Varnish, GC) and casein phosphopeptides-amorphous calcium phosphate (CPPACP) pastes (Tooth Mousse, GC) were applied to retard caries progression and remineralize dental tissue (2). Remineralization and restoration of cervical lesions were carried out to maximize the retain tooth structure more conservative and less invasive (3) with the use of flowable composite (G-aenial Flo, GC) and glass ionomer (Equia, GC). The patient was re-evaluated every 6 month (4) for the maintenance of oral status.

Results: Minimally invasive approach to restorative dental care would appear to be appropriate for older adults. 
Conclusion: MID offers the dental professional working with older patients realistic, rational, evidence based options for treating oral disease.

\section{CC16}

\section{A fiber rein-forced composite bridge for missing maxillary lateral} incisor

Ali Alabas, Elif Aybala Oktay, Fulya Toksoy Topcu Gulhane Military Medical Faculty, Department of Restorative Dentistry, Ankara, Turkey

Objective: The people with missing lateral incisors must be treat quickly to restore function and aesthetics. The loss of anterior teeth can be painful for patients, both psychologically and socially. A variety of a treatment options, from implant to conventional bridge prosthesis, can be used for the replacement of a missing anterior tooth. The fiber reinforced composite bridges are good alternative to conventional prosthetic techniques because of being a non-invasive or minimally invasive application with very little or no tooth preparation.

Case Report: A 22-year-old young woman was referred to our clinic for her missing maxillary left lateral tooth. We have decided to apply fiber rein-forced bridge to fill the gap.

Result: The treatment was completed in a one-visit appointment.

Conclusion: A fiber rein-forced composite bridge is generally less costly and provides immediate esthetic solutions then traditional prosthetic options. The anatomical layering technique provides naturel opacity, transluceny and opalescence with using of different dentin and enamel composites to build up the composite pontic. But composites are brittle materials. Fiber reinforcing of a composite resin bridge increases the fracture toughness and resistance.

\section{CC17}

\section{Aesthetic approach for bilateral peg shaped maxillary lateral incisors}

Ali Alabas, Elif Aybala Oktay, Fulya Toksoy Topcu Gulhane Military Medical Faculty, Department of Restorative Dentistry, Ankara, Turkey

Objective: Today, people especially young adolescents are paying more attention to their appearance ever before and their teeth are a key element in their personal appearance. Modern adhesive techniques are now used to add restorative materials to the tooth for the correction of unaesthetic tooth forms, tooth positions, dimensions, colors, and to close diastemata, to build out peg-shaped laterals and to change the tooth form of elements with an abnormal anatomical position.

Case Report: This clinical report describes aesthetic and preventive approaches for treatment of a patient diagnosed with bilateral peg-shaped lateral incisors. Direct composite veneers were applied to this case.Natural Look DFL (Rio de Janeiro, RJ, Brazil) composite laminate in B1 color together with 37\% orthophosphoric acid Alpha Etch Gel and Alpha Bond (Rio de Janeiro, RJ, Brazil), all from the composite kit, were used for this purpose
Result: An 18 years old girl has begun to smile easily without caring her teeth appearance.

Conclusion: Conical tooth anomaly is a hereditary and otosomal dominant disorder which usually effects the maxillary permanent lateral incisors and third molars. Generally, the affected teeth are smaller than normal sizes and usually show a conical form which may be peg, cylindrical, barrelshaped etc. This anomaly can give rise some physiological and psychological problems. Because of this reason, early diagnosis and treatments are important. For treatment of this anomaly, composite veneer restorations can be made and thus an ideal oral condition and pleasure of patient may be provide esthetically and functionally.

\section{CC18}

Treatmant of flourosis and enamel hypoplasia with direct laminate veneers

Ali Alabas, Elif Aybala Oktay, Fulya Toksoy Topcu

Gulhane Military Medical Faculty, Department of Restorative Dentistry, Ankara, Turkey

Objective: The defects caused by enamel hypoplasia and the discolorations caused by dental flourosis that occur at the anterior teeth can cause aestetic problems. Because of aesthetic problems and decay risc, such cases must be urgently treatment. For the treatment of these cases ,laminate veneer restorations may be a treatment option.

Case Report: In these two cases, enamel hypoplasia and flourosis was observed at two patients that presented to our clinic. The advantage of aesthetic, simply use and short treatment time, direct composite resin restoration application was decided to both patients.Natural Look DFL (Rio de Janeiro, RJ, Brazil) composite laminate in A1 color together with $37 \%$ orthophosphoric acid Alpha Etch Gel and Alpha Bond (Rio de Janeiro, RJ, Brazil), all from the composite kit, were used for this purpose

Result: As a result of direct composite resin application, reached an acceptable aesthetic.

Conclusion: Enamel hypoplasia and flourosis, result in esthetic and even psychological problems. At the treatment of enamel hypoplasia and flourosis, from simple to complex tooth colored restorative materials, laminate veneer restorations and all ceramic restorations can be used. It should be aimed to choose the less harmful, the quickest and the most esthetic treatment method. Direct composite veneers are thought to be more conservative than prosthetic approaches

\section{CC19}

Direct laminate veneers instead of orthodontics treatment

Ali Alabas, Elif Aybala Oktay, Fulya Toksoy Topcu Gulhane Military Medical Faculty, Department of Restorative Dentistry, Ankara, Turkey

Objective: A few people are born with a perfectly aligned smile. If your teeth are crooked or misaligned,you may need to use orthodontics to improve the appearance of malposed teeth. However, some people are not enthusiastic about the idea of wearing fixed braces for an extended period of time. Furthermore, composite veneers can be used to fix minor misalignments in just a single appointment. 
Case Report: In these two cases, class one malocclusion was observed at two patients that presented to our clinic. In accordance with the wishes of the patients, direct composite veneers were applied. Natural Look DFL (Rio de Janeiro, RJ, Brazil) composite laminate in B1 color together with 37\% orthophosphoric acid Alpha Etch Geland Alpha Bond (Rio de Janeiro, RJ, Brazil), all from the composite kit, were used for this purpose.

Result: We have created the appearance of a straight, white, aligned smile.

Conclusion: The advantages of using direct laminate veneers to restore the teeth are many. In a short time very good aesthetics can be obtained, with minimal tooth preparation. And also they are becoming more popular as they are easy to repair. However the main disadvantages of them are discoloration, fractures and low resistance to wear. Furthermore, indirect composite laminate veneer restorations have better color stability due to polymerization outside of the oral cavity.

\section{CC20}

\section{Some little aesthetic touches with composites to make you happy}

Ali Alabas, Elif Aybala Oktay, Fulya Toksoy Topcu Gulhane Military Medical Faculty, Department of Restorative Dentistry, Ankara, Turkey

Objective: Defects in the maxillary anterior teeth, such as fractures in teeth crowns, can present esthetic challenges. A treatment plan that can be completed in a single appointment is highly desirable such as these cases. Laminate veneers are restorations which are envisioned to correct existing discolorations ,abnormalities and esthetic deficiencies. Direct laminate veneers have no need to be prepared in the laboratory and are based on the principle of application of a composite material directly to the prepared tooth surface in the dental clinic.

Case Report: A 21-year-old female with incisal edge fractures of maxillary central incisor and a 26-year-old female with enamel and dentin fractures of both maxillary central incisors were observed at two patients that presented to our clinic. Direct composite veneers were applied to both of them. Natural Look DFL composite laminate in A1 color together with $37 \%$ orthophosphoric acid Alpha Etch Gel and Alpha Bond, all from the composite kit, were used for this purpose

Result: The final result met the patient's expectations.

Conclusion: Usually because of difficulties with the final restoration of the treatment of fractured teeth, can be complicated. The systematic history and examination are very important in all cases of dental trauma treatments. It also shows that conservative management is possible or not .Direct and indirect laminate veneers, as esthetic procedures, have become treatment alternatives for patients with esthetic problems of anterior teeth in recent years. In deciding between treatment options, the cost, social and time factors have to be considered.

\section{CC21}

\section{Only 30 minutes is sufficient for whiter teeth}

Ali Alabas, Elif Aybala Oktay, Fulya Toksoy Topcu Gulhane Military Medical Faculty, Department of Restorative Dentistry, Ankara, Turkey
Objective: Today beautiful smile with white teeth is one of the most important expression of a person. Nothing could be more desirable than a beautiful and white smile. Aesthetic dentistry, including tooth whitening, has increased in recent years. Dental bleaching is more conservative, quick, simple, and low cost approach to change the color of discoloured teeth. Vital tooth bleaching can be used to fix discolored teeth in just a single appointment.

Case Report: In these two cases, discoloration was observed at two patients that presented to our clinic. In office vital bleaching technique were applied for thirty minutes to get whiter teeth for both cases. As bleaching agent, Whiteness HP (FGM, Joinville, Brazil), containing $35 \%$ hydrogen peroxide was used.

Result: After 30 minutes bleaching treatment, whiteness was obtained which satisfied the patient.

Conclusion: In-office systems, typically $35 \%$ hydrogen peroxide whitening agent are used. Advantages include minimal dependence on patient compliance and immediate visible results, which satisfy patients who want to see quick results . Tooth sensitivity is the most common side effects in vital tooth bleaching. Usually the sensitivity was moderate and was more severe on the day of the gel application. With the addition of potassium nitrate-fluoride into bleaching agent, reduces sensitivity of bleaching.

\section{$\mathrm{CC22}$}

\section{Nonvital bleaching is enough to get a better color}

Ali Alabas, Elif Aybala Oktay, Fulya Toksoy Topcu Gulhane Military Medical Faculty, Department of Restorative Dentistry, Ankara, Turkey

Objective: In recent years, number of patients admitting to the dentist for a beautiful smile is increasing. Discolorations of nonvital teeth lead to esthetic problems since they can be easily distinguished in teeth. The reason for this is that they are usually asymmetrical. So they need to be treat.

Case Report: In this clinical study, 2 patients were treated using carbamide peroxide for intracoronal bleaching of maxillary santral incisor teeth discolorated due to trauma and root canal therapy. As bleaching agent, Whiteness Super-Endo (FGM, Joinville, Brazil), containing 37\% carbamide peroxide was used.

Result: Bleaching in both two cases was achieved in two days. The treatment with carbamide peroxide as regards the handling and application time proved advantages.

Conclusion: Discoloration may result from pulp necrosis, intrapulpal hemorrhage, pulp residues remaining after endodontic treatment, endodontic materials, resorption and aging for the treatment of non-vital teeth, the most conservative one is intracoronal bleaching technique. Most commonly used bleaching agent is Hydrogen Peroxide. The bleaching agents on the market generally contain carbamide peroxide(CP) or hydrogen peroxide(HP). CP solutions do not have a stable structure and they decompose to HP, carbon dioxide and urea in oral environment. Then bleaching process occurs with HP 


\section{$\mathrm{CC24}$}

Case series demonstrating poly-ether-ether-ketone (PEEK) framework RPDs in the rehabilitation of partially dentate patients.

\section{Zaid Ali ${ }^{1,2}$, Nicolas Martin ${ }^{1,2}$, Sarah Baker ${ }^{1}$ \\ ${ }^{T}$ University of Sheffield, Sheffield, UK, ${ }^{2}$ Charles Clifford Dental Hospital, Sheffield, UK}

The use of poly-aryl-ether-ketones (PAEK) materials as metal alternatives in the aerospace, device manufacturing and medical fields has been in place now for over twenty years. The introduction of these materials to the dental market is a novel development in the last few years. Market driven and patient led pressures to replace non-precious metal materials in prosthetic dental devices may encourage clinicians to take up the use of this novel material in their clinical practice.

The proposed advantages of PAEK polymers as non-metal framework alternatives are, in addition to being non-metal; having a modulus of elasticity closer to that of bone thereby reducing stresses to abutments, high wear resistance, high strength-to-weight ratio, manufacturing predictability and a proven track record for biocompatibility.

One such PAEK polymer which has been CE marked for use in dentistry is the JUVORA ${ }^{\mathrm{TM}}$ Dental Disc. This is a poly-ether-ether-ketone (PEEK) disc that is milled to a digitally designed shape using either a scan of a working model or data from an intra-oral scan impression.

We present three cases of the use of the Juvora dental disc as a removable partial denture material in the rehabilitation of partially dentate patients treated at the Charles Clifford Dental Hospital, University of Sheffield. Early outcomes are considered and discussed.

\section{$\mathrm{CC25}$}

\section{Dental inlays using nano-hybrid composite. Do it yourself}

Sebastiana Arroyo Bote, Javier Martinez Mmartinez Osorio

Faculty of Dentistry Barcelona University, Barcelona, Spain

Objective: The aim of the study was to perform laboratory-free dental inlays using Nano-Hybrid Composite. (GrandioSO, VOCO)

Introduction: Increased life expectancy of our patients obliges us to perform minimally invasive reconstructive treatments and to using materials that offer the best properties. This study presents two clinical cases where the clinicians doing dental inlays without laboratory

Clinical Cases: Two patients with coronal destruction of 4.6, one vital and another endodontically treated.

Treatment begins with carious tissue removal, cleaning and cavity preparation. In the vital case, we perform a direct impression of the cavity. For the endodontically treated case, a cavity floor is made in the distal conduit to place a fiberglass pole. After testing and adjusting the post an impression is made

In both cases, we empty the prints and perform Nano-Hybrid Composite (GrandioSO, VOCO) inlays using layering technique and 20-second polymerization cycles. We perform another 120 -seconds polymerization cycle on the inner part of the inlays.

Finally, we model and polish the inlays and, after clinical test, we perform adhesive bonding and occlusal adjustment of the inlay.

Discussion-Conclusion: Dental reconstruction is one of the most important treatments in Conservative Dentistry because of the technical challenge of rebuilding the crown and the importance of the materials we use for it. Indirect treatment allows for a better handling and polymerization of composites, helping to improve their properties.

\section{$\mathrm{CC26}$}

In case of one tooth absence, discoloration, and mobility, restorative treatment

Ertürk Bilgec,, Fulya Toksoy Topçu, Elif Aybala Oktay

Gulhane Military Medical Faculty, Department of Restorative Dentistry, Ankara, Turkey

The patients are gradually requesting to complete their procedures in one session. Although this request is not feasible in all procedures, thanks to fibre technology, one session bridge production has become available for ruling out the one tooth lack. In order for eradicating one tooth lack, it could be used in mobile teeth as splint, as well. In this case report, one tooth lack was eradicated with adhesive bridge strengthened with fibre while it was used as a fibre splint to reduce the mobility in the teeth of this region. The decays and discolorations of the anterior teeth were treated with composite laminate veneer.

49 year old male patient presenting with tooth decays, discoloration and lack of the right upper lateral tooth, referred to our clinic .In order for ruling out the tooth lack(Angelus Interlig, Londrina, PR, Brasil) glass fiber (Technical \& General, London, England) flowable composite,A2 colored (Herculite XRV, Kerr, Scafati, Italy) composite and(Alpha Etch Jel, Rio de Janerio, RJ, Brazil) 37\% orthophosphoric acid and (Optibond, Kerr, Scafati, Italy) bonding were used for bridge application. The fibre was fixed to the mobile teeth and used as splint. Composite laminate veneer restoration was performed on the anterior teeth with the same composite set.

Due to the fact that teeth, alveolar bones were inappropriate for implant or crown bridge prosthesis procedure, we tried to perform the procedure with the adhesive bridge strengthened with fibre. Due to the dental mobility, the fibre was also used as a splint. With the composite laminate veneers, the patient gained his good appearance. Such procedures provide an aesthetic and advantaged restoration to the dentists thanks to the features including economic, adhesive, and aesthetic, one session applicable, splint use, biocompatible and easy to repair.

\section{$\mathrm{CC27}$}

\section{Quick and alternative solutions for aesthetic expectations}

Ertürk Bilgec, Elif Aybala Oktay, Fulya Toksoy Topçu

Gulhane Military Medical Faculty, Department of Restorative Dentistry, Ankara, Turkey 
With the introduction of developing fibre technology in the dentistry, one tooth lack recovery has become easy within a short period. Adhesive technology provided an economic restoration and it became an alternative to conventional methods. In this case report, it is reported that adhesive bridge strengthened with fibre for one tooth lack as well as discoloured and former restorations containing upper anterior teeth were exposed to composite laminate veneer restoration procedure.

38 year old female patient presenting with lack of right upper lateral tooth and discoloration in the teeth referred to our clinic. She reported that she had little time, and due to the economic reasons she requested us not to apply prosthesis or implant. As a result of intraoral and radiographic examination of the patient, adhesive bridge strengthened with fibre was used for eradicating the lack of tooth, and for dental discoloration and former restorations, composite laminate veneer procedure was decided to be applied. In order for ruling out the tooth lack (Angelus İnterlig, Londrina, PR, Brasil) glass fiber (Technical \& General, London, England) flowable composite, A2 colored (Herculite XRV, Kerr, Scafati, Italy) composite and (Alpha Etch Jel, Rio de Janerio, RJ, Brazil) $37 \%$ orthophosphoric acid and (Optibond, Kerr, Scafati, Italy) bonding were used for bridge application and composite laminate veneers.

Adhesive bridges strengthened with fibre are economic, aesthetic, one session applicable, biocompatible and easy to repair provide the advantaged restoration to us.

\section{CC28}

\section{Change the appearance in a session}

Ertürk Bilgeç, Fulya Toksoy Topçu, Elif Aybala Oktay Gulhane Military Medical Faculty, Department of Restorative Dentistry, Ankara, Turkey

In today's dentistry, patients want the processes without pain and in a short time. These requests have led the dentists to the short-term treatments Developments of composites present to dentists to make good restorations in a short time. In that case report, the esthetic problems solved in a session on the upper anterior teeth with caries and discolorations.

23 year old young male patient presenting caries and discolorations on upper anterior teeth referred to our clinic. $\mathrm{He}$ complained from the appearance. He can't smile comfortable. As a result of intraoral and radiographic examination of the patient composite laminate veneer procedure was decided to be applied. First we treated the caries then we prepared teeth for direct laminate veneer. We choosed the convenient colours as dentin and enamel and made the direct laminate veneers. A2 colored (Herculite XRV, Kerr, Scafati, Italy) composite and (Alpha Etch Jel, Rio de Janerio, RJ, Brazil) 37\% orthophosphoric acid and (Optibond, Kerr, Scafati, Italy) bonding were used for composite laminate veneers

The bonding agent systems and composites present us to restore teeth without microleakage and we can make good colour on teeth. The patients are happy about restoration finished in a session and good appearance.

\section{CC29}

\section{An esthetic bridge in a session}

Ertürk Bilgec,, Elif Aybala Oktay, Fulya Toksoy Topçu

Gulhane Military Medical Faculty, Department of Restorative Dentistry, Ankara, Turkey

Nowadays the dentists work fast and less harmful to answer the patients' expectations. Lack of tooth can be resolved with prothesis and implant but these treatment can't be made in a session. The fibre technology solve the time problem. In this case report, lack of the right upper canin tooth problem was solved with the fibre reinforced bridge in a short time.

36 year old female patient presenting with lack of right upper canin tooth referred to our clinic. She reported that she had little time, and due to the economic reasons she requested us not to apply prosthesis or implant. As a result of intraoral and radiographic examination of the patient,adhesive bridge strengthened with fibre was used for eradicating the lack of tooth. In order for ruling out the tooth lack (Angelus Interlig, Londrina, PR, Brasil) glass fiber (Technical\&General, London,England) flowable composite, A2 colored (Herculite XRV, Kerr, Scafati, Italy) composite and (Alpha Etch Jel, Rio de Janerio, RJ, Brazil) 37\% orthophosphoric acid and (Optibond, Kerr, Scafati, Italy) bonding were used for bridge application. So we solved the patient's functional and esthetic problem in a session with the fibre reinforced bridge.

We used fibre in the bridge because of the patient's requests. This bridge technique isn't stronger than classic prothesis. But Adhesive bridges strengthened with fibre are economic, aesthetic, one session applicable, biocompatible and easy to repair provide the advantaged restoration to us.

\section{CC30}

\section{Minimal invasive treatment to white spot lesion}

Ertürk Bilgeç, Fulya Toksoy Topçu, Elif Aybala Oktay

Gulhane Military Medical Faculty, Department of Restorative Dentistry, Ankara, Turkey

Nowadays, people are getting more and more worried about esthetism. While solving the esthetic problems, it is crucial that the patients' teeth are given as less harm as possible. If patients' discoloration problems cannot be solved by using bleaching techniques, the dentists use restorative ways. In this case report the upper four anterior teeth are restored with composite after minimal preparation.

27 year old female patient presenting with tooth discoloration and cavities referred to our clinic. The patient stated that she didn't want any prosthetic restoration. After examining the patient, it was easily seen that the discoloration couldn't be removed with bleaching methods. We decided that the teeth was restored with composite to get the esthetism again. A2 colored (Herculite XRV, Kerr, Scafati, Italy) composite and (Alpha Etch Jel, Rio de Janerio, RJ, Brazil) 37\% orthophosphoric acid and (Optibond, Kerr, Scafati, Italy) bonding were used for composite laminate veneers. 
It was a good choice to prefer to restore the teeth with the composite due to the fact that the discoloration cannot be treat with bleaching and it will be harm to make prosthesis. To become better with the developing technology of composite offers opportunities for minimally invasive interventions to dentists. Therefore the patients' satisfaction increases.

\section{CC31}

A resin composite layering approach for solving complex class IV restorations: a case report.

Romain Ceinos $^{1,2}$, Marie-France Bertrand ${ }^{1,2}$

${ }^{T}$ Nice Sophia Antipolis University, UFR Odontologie, Department of Conservative Dentistry and Endodontics, Nice, France, ${ }^{2}$ Nice University Hospital, Pôle Odontologie, Nice, France

Objective: A 60-year-old patient consulted the Nice university hospital to get a cosmetic opinion. The aesthetic problems on maxillary incisors were complex and linked to fractured and decayed teeth, old infiltrated or misshapen composite resin restorations. The treatment involved the replacement of faulty restorations. The two options for restoring teeth were discussed with the patient:

- a direct composite layering, the benefits of which include easy repair and a more conservative approach

- an indirect restoration made-up by a ceramist, which provides greater color homogeneity.

The patient rejected this high-cost option and desired smile enhancement in the most minimally invasive manner.

Methods: Study casts were taken and mounted on a semi-adjustable articulator to achieve an aesthetic analysis. A diagnostic wax-up was carried out to simulate the teeth's shape and volume modifications. The patient accepted the treatment plan. Direct anterior restorations were made using a layering technique with Enamel Plus HRi ${ }^{\circledR}\left(\right.$ Micerium $\left.^{\circledR}\right)$ and special attention was given to finishing adjustment. The procedure was performed under rubber dam isolation to ensure the control of moisture.

Results: The four maxillary incisors were restored with a natural look. The patient confirmed her satisfaction with the smile enhancement.

Conclusion: The aesthetic integration of a restoration remains a complex challenge especially as far as the anteriors are concerned. As composite resins are used for aesthetic enhancement, the procedure represents a valuable framework on which to develop a rich stratification of natural teeth colors that achieves the expectations of both patient and practitioner.

\section{CC32}

Additive restorations of anterior teeth: improvement of treatment planning trough Aesthetic Digital Smile Design

Riccardo Tempesta

University of Turin, Turin, Italy

Objectives: This report deals with one case of additive restorations on anterior teeth to close diastemas and to modify teeth shape. The aim of this case report is to show the importance of Aesthetic Digital Smile Design (ADSD) in treatment planning.

Methods: The patient was a 19-years-old female that decided to stop a complex treatment after 4-year. The upper anterior region presented 2.2 conoid and diastemas between 1.1,1.2 and 2.1. The initial treatment, planned by the orthodontist, provided the shape correction of 2.2. However, once the patient was visited in the Operative Dentistry Department, the patient was involved in the treatment planning with ADSD, that through a dento-facial analysis performs a digital project of the aesthetic treatment. The final aesthetic result chosen by the patient was realized on a gypsum model with wax, thus the dentist could obtain several silicon guides that are needful to replicate the ADSD result. Additive restorations were then performed following a standardized clinical procedure: field isolation through rubber dam; teeth cleaning with aluminium oxide sandblasting; etch-and-rinse adhesive system application; direct additive restorations were performed using Clearfil-ES2 in an incremental layering technique with the help of silicon guides; interproximal contacts were created with shaped acetate matrices.

Results: 7 days and 12 months follow-up showed perfect aesthetic result and complete patient satisfaction.

Conclusions: Direct additive restoration can be a conservative choice for diastemas closure and tooth shape modification. Nowadays ADSD is fundamental to decide with patient its aesthetic restoration and to guide the dentist in performing it.

\section{CC33}

Open apex necrotic treatment options. Revitalization versus MTA apical barrier.

Cristina González Losada ${ }^{1}$, Alfredo Saralegui Calvo ${ }^{1}$, Vicente Vera González

${ }^{1}$ Complutense University, Madrid, Spain, ${ }^{2}$ Complutense University, Madrid, Spain, ${ }^{3}$ Complutense University, Madrid, Spain

Introduction: Calcium hydroxide has been considered the gold standard when choosing a material to induce apical root closure. After Andreasen publication's about decreased resistance to fracture of immature teeth after filling with calcium hydroxide, other treatment options had to be considered.

Actually open apex treatments are MTA apical barrier or "Revascularization technique", described by Trope, also named "Revitalization".

Clinical cases: We show different treatment options depending on the apex size and the age of the patient.

Case 1: Central incisor, ten years old patient with a non-vital immature teeth cylindric canal, not wider than $1,1 \mathrm{~mm}$. In this case an apical MTA barrier was created to seal the apex successfully.

Case 2: Eight years old patient with a central incisor traumatically injured. The open apex is wider than the case described before eight. Better option to indicate a "revitalization".

Case 3: Forty years old woman with open necrotic reabsorbed apex. An apical barrier with MTA was indicated due to age of patient and its apical anatomy. 
In all these clinical cases radiolucencies were healed and clinical symptoms were resolved but only in Case 2 roots continued to develop.

Conclusions: Open apex necrotic teeth options are the creation of an apical barrier, the revitalization technique or both in case of failure of the last option.

Revitalization must be considered the preferred option in recent days as it can help to improve the prognosis of necrotic immature teeth by strengthening dentinal walls. It reduces risk of root fracture for now.

\section{$\mathrm{CC34}$}

\section{Conservative dentistry in provisionalization and progressive loading}

Javier Martinez Osorio, Sebastiana Arroyo Bote, Esther Lazaro, Esther

Berastegui Jimeno

Faculty of Dentistry. Barcelona University, Barcelona, Spain

Objectives: The aim of this study is to show the use of composites, glassfiber reinforced with composite, adhesives and resin cements in provisionalization and progressive loading in patients undergoing dental rehabilitation.

Clinical Cases: Clinical cases of direct clinical reconstruction in patients with aesthetic and functional needs are presented. We perform rehabilitations of teeth within the incisor canine group using glass fiber-reinforced composite. The different opacities and effects of composites are particularly suitable for leadingedge aesthetics to treat urgent problems and/or when we need time to work on a definitive treatment.

We used conservative materials in the rehabilitation of a patient who had lost an anterior tooth in a traumatic situation. In the moment of the extraction we replayed the tooth using conservative techniques and materials. This treatment maintained the aesthetic appearance of the patient during the period in which the tissues healed. With another patient we used these materials during the period of osseointegration of implants to maintain aesthetic appearance and apply progressive occlusal forces over the implant.

Discussion and Conclusions: The aesthetic needs of today's society require us to provide immediate solutions for patients. The aesthetic adhesive materials allow the clinician to restore the aesthetic function of teeth immediately. The patients can have these treatments for a relatively long period in order to give sufficient time to study each case and provide definitive solutions. Composites with different densities, adhesives, glass-fiber reinforced with composite, and resin cements are suitable to solve these aesthetic cases.

\section{$\mathrm{CC35}$}

Tridimensional Radiology applied to the treatment of calcified canals in Endodontics

Cristina González Losada ${ }^{1}$, Alfredo Saralegui Calvo ${ }^{1}$, Paula Saralegui González

${ }^{1}$ Complutense University, Madrid, Spain, ${ }^{2}$ Complutense University, Madrid, Spain, ${ }^{3}$ Autonoma University, Madrid, Spain
Introduction: $\mathrm{CBCT}$ is a 3- $\mathrm{D}$ technnology device, derived of Computer Tomography technology, whose main advantages are its reduced economic cost and radiation exposure.

Endodontic therapy depends on diagnosis radiographs and image guided treatment. Periapical and panoramic radiography have been supplemented by the introduction of limited field of view ( FOV) high resolution CBCT, allowing three dimensional assessment of lesions, root canal morphology and anatomy.

Periapical radiographies allow mesio-distal adjustments during endodontic treatment but if we need confirmations of the canal position in a sagital ( vestibule - palatal axis) we must resort to the use of $3 \mathrm{D}$ images.

Clinical cases: We show two clinical cases of calcified canals which made necessary an intraoperatory CBCT (Kodak 9000) to locate the canal position. In both patients periapical radiographies were not enough to place its position in a sagital axis, so a further tridimensional image testing was necessary to stablish a proper and accurate diagnosis.

Sequence or conventional and tridimensional images are describes in both cases.

Conclusions: Use of CBCT in Endodntics is limited to complex clinical cases when conventional radiography methods do not allow clinicians to establish a concludent diagnosis. This device is also useful during the endodontic treatment, especially in case of calcified canals when the determination of canal spatial situation is necessary.

This technique offers the possibility to analyze multiple section of the tooth in three planes so it helps to interpret and understand images and avoids the risk of perforations during the canal location phase.

\section{CC36}

\section{Subcutaneous emphysema as a complication of endodontic treatment.}

Fawad Amin

Bristol Dental Hospital, Bristol, UK

Background: Subcutaneous emphysema is rarely encountered in endodontic practice and consequently there is a dearth of information in the dental literature about this complication.

Subcutaneous emphysema is defined as the abnormal presence of air in tissue spaces. It arises when air is forced, under pressure, into the subcutaneous fascia leading to a sudden onset of soft tissue swelling. Introduction of air via the air-driven hand piece during surgical removal of impacted teeth is the most common cause of subcutaneous emphysema. Less commonly, it results from nonsurgical dental procedures including root canal treatment.

In most cases emphysema during root canal therapy resolves spontaneously after few days. However, it can be a frightening and unpleasant experience for both patient and dentist.

Observations: During re root canal treatment of the UR1, the patient experienced discomfort and swelling around her right 
eye. This eventually resulted in her being unable to open her right eye. Most probably compressed air from the air syringe entered through the patent canal and spread along the facial planes into the periorbital space.

She was referred to an ophthalmologist who confirmed the diagnosis of periorbital emphysema. The patient was successfully treated with oral antibiotics and made an uneventful recovery.

Conclusions: Dentists should be aware of subcutaneous emphysema and its presentation. It is characterised by sudden onset of soft tissue swelling, associated with crepitus, during or shortly after the procedure. The majority of cases are managed conservatively. Patients should be reassured and advised as to the nature of emphysema.

\section{$\mathrm{CC} 37$}

A clinical complication of fiber-reinforced ribbon-composite provisional fixed partial denture of an adolescent patient

Derya Merve Halacoglu, Duygu Tuncer, Burcu Oglakci, Neslihan Arhun Baskent University, Ankara, Turkey

Background: Treatment of adolescent patients with a missing incisor may be compromising because implants cannot be placed until craniofacial growth is complete. Fiber-reinforced ribbon-composite fixed provisional partial dentures can be used for the replacement of the missing teeth. Since the craniofacial growth continues in transverse, sagittal and vertical directions, regular control appointments must be managed to check the alveolar ridge/gingival level in the pontic area. This case report describes a clinical complication of fiber-reinforced ribbon-composite fixed provisional partial denture of an adolescent patient with a trauma originated missing incisor.

Materials and Methods: A 14 years old male patient who had attended to Baskent University in 2010, had a traumatic dental injury in \#21 tooth which was extracted. Afterwards the missing tooth was replaced with a fiber reinforced ribbon-composite fixed provisional partial denture with patient's tooth as pontic until the definitive implant supported single-tooth prosthetic rehabilitation and the family was asked to visit every six months regularly.

Results: The patient didn't attend to the control appointments until the provisional denture's failure and the harmony between pontic tooth and the alveolar ridge/gingiva was severely compromised after 4 years.

Conclusion: If the goals of treatment favors fiber-reinforced ribbon-composite fixed provisional partial denture use before skeletal maturation, attention must be given to prosthesis design. Regular control appointments of alveolar ridge and pontic design must be managed and pontic tooth must be re-shaped to accommodate physiological soft and hard tissues in accordance with the skeletal growth to ensure esthetic future single implant supported prosthetic rehabilitation.

\section{CC38}

\section{Management of the fractured and dehydrated tooth fragment}

Derya Merve Halacoglu, Duygu Tuncer, Neslihan Arhun Baskent University, Ankara, Turkey
Reattachment of the fractured and dehydrated tooth fragment should be considered as a first treatment choice and it is a valid alternative to a composite resin restoration. This case report presents the restoration of a fractured maxillary central incisor by reattaching the dehydrated tooth fragment.

A 17 years old male patient attended to the Baskent University, Restorative Dentistry department with the complaint of the fractured tooth (\#11) and the fractured tooth fragment was kept in dry conditions for 5 days. After clinical and radiographic examinations, discolored composite resin restorations were noticed on \#11 and \#21. The fractured tooth and the fragment were acid etched with $\% 37$ phosphoric acid and then rinsed with water, dried gently. The bonding agent (Adper Single Bond-2, 3M ESPE) and flowable composite resin (Clearfil Majesty-A2, Kuraray) were applied without light curing on fractured tooth and fractured fragment. The fragment was carefully repositioned to the fractured tooth gently with finger pressure and light cured for 20 s twice. After reattaching the fragment, discolored composite resin restorations were removed and new restorations were done with the same adhesive system and a nanohybrid composite (Spectrum TPH-A2, Dentsply) and finished-polished with finishing/polishing disks (Sof-Lex, 3M ESPE). Finally the reattached fragment was whiter than tooth but the white color was compensated after 1 week service in the mouth by rehydration. Restoration of the tooth by reattaching the original dehydrated fragment and gaining its natural color on the mouth is the best way of treatment in esthetic, conservative and economic point of view.

\section{CC39}

Conservative treatment of patients received head and neck radiation therapy - Protocols and clinical cases

Angeliki Karveli, Elissavet-Maria Tagka, Dimitris Kambouropoulos, Afrodite Kakaboura

Dental School of Athens, Athens, Greece

Patients, who receive anti-neoplasmatic therapy, usually present symptoms in the oral cavity such as oral mucositis, xerostomia and dental caries. Concerning dental caries, these patients are considered as high-risk patients. The management of these patients should begin before the therapy with treatment of all the dental problems and with intense preventive protocols, which are customized according to the type and phase of their antineoplasmatic therapy. Lastly, a strict recall system during and after the therapy is followed in order to monitor the changes in the oral cavity and deal with possible implications. However, even after application of this protocol, excessive loss of dental tissues due to caries is a common phenomenon. In addition, severe trismus due to surgical procedures or fibrosis of the masticatory muscles often reduce the mouth opening and further complicate the treatment procedures of the dental lesions. Furthermore, due to their serious health problems, a high percentage of such patients are left without specific dental care. As a result, extensive dental caries cause remarkable difficulties for tooth restoration. Even more, technically easy, less time-consuming and low-cost treatment plans are necessary for the most of the patients described.

In the current study, specific protocols regarding the prevention of dental caries which should be applied before, during and after the antineoplasmatic therapy will be reported. Furthermore, the step by step clinical conservative approach with resin composites for tooth rehabilitation in representative cases of patients who received anti-neoplasmatic therapy and were suffering from extensive dental caries will be presented. 


\section{CC40}

A magical touch of cryotheraphy on oral leukoplakia, a case report Sencer Secer ${ }^{1}$, Didem Dincer ${ }^{2}$, Hilal Ozturk ${ }^{3}$, Necdet Dogan ${ }^{1}$, Hasan $\overline{\text { Ayberk Altug }}^{1}$, Metin Sencimen ${ }^{1}$, Tamer Zerener ${ }^{1}$

${ }^{1}$ Oral and Maxillofacial Surgery Department, Gulhane Military Medical Academy, Ankara, Turkey, ${ }^{2}$ Dermatology Department, Gulhane Military Medical Academy, Ankara, Turkey, ${ }^{3}$ Oral and Maxillofacial Radiology Department, Gulhane Military Medical Academy, Ankara, Turkey

\begin{abstract}
Aim: Oral leukoplakia (OL) is a relatively common and a potentially malignant white lesion of the oral cavity. Tobacco and alcohol misuse are significant risk factors for the development of OL. The presence and degree of dysplasia are important diagnostic and prognostic criteria for OL. There is a lack of consensus on the most appropriate method of OL management. Cryotherapy is a frequently used method with promising results, that locally destroys lesion tissues by freezing in situ. In this case we aimed to show the steps of healing OL with cryotherapy.
\end{abstract}

Case: A 48 years old female heavy smoker patient was admitted to our clinic with a painless but a few stinging white plaque like lesion on all over her lower side of tounge and lingual side of alveolar crest between molars. An incisional biopsy was done on the lingual area and the patological report was orthokeratotic type leukoplakia with mild degree dysplasia. Complete excision was not possible due to the damage risk on salivary gland, lingual nerve and vessels, than we decided to apply cryotherapy.

Results: After cryotherapy treatment which was maintained for 9 months the lesion was disappeared without any complaint and any possible surgical complications.

Conclusion: Complete excision of high-risk lesions is recommended in suitable cases, besides careful selection of patients, cryotherapy is a simple, safe, easy, conservative, and acceptable treatment modality for treating OL. Specialists should closely follow up these patients with OL for life, like as we are going to do in this case.

\section{CC41}

Restorative Clinical Case (Conservative, Periodontics, Oral Surgery, Prosthodontics)

Steve Conteh

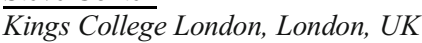

A 31 year old male attended the Primary Dental Care Clinic as a new patient for an oral health assessment 3-4 years since his last oral health assessment in the UK.

Upon comprehensive history taking, clinical examination and special testing the patient was diagnosed with:

- Generalised Moderate Chronic Periodontitis

- Dentine hypersensitivity most likely from TSL Tissue Surface Loss (TSL)

- Erosion + Attrition of maxillary anterior teeth-possibly due to Gastro-Oesophageal Relux Disorder (GORD) and reduction in posterior support
- Caries in UR7(mesial) UR3(distal) and UL5(mesial) Secondary Caries (UR5 and 6)

- $\quad$ Fractured LL5(distal), UR 5 and 6(occlusal)

- Unrestorable retained roots (UL4 and UR4)

A plan was put in place to first acheive stabilisation from active pathology. This involved significant prevention and intervention.

Having achieved stabilisation in dental health, through repeated preventative and interventive means, we were able to discuss definitive treatment option. All options appropriate to this case were discussed, together with their risks, benefits, length of treatments and what to expect. The patient was also informed of the consequence of no treatment.

We came to a suitable agreement on the definitive treatment to be embarked upon.

Definitive treatment was completed with a very favourable outcome that both the patient and I were very pleased with. The patient will continue regular periodontal maintenance of a few sites with appropriate changes being made to recall and intervention as indicated by examination result.

I am most pleased with the improvement in periodontal health as we would not have been able to build on pathological foundations. Work completed by myself with nurse assistance..

\section{$\mathrm{CC42}$}

Management of severe tooth wear and occlusal collapse using composite build-up and overdenture

Hayder Ameer ${ }^{1,2}$

${ }^{7}$ Guy's and St Thomas' NHS Trust, London, UK, ${ }^{2}$ Peninsula Dental School, Plymouth, UK

This is a clinical presentation illustrating the restorative management of a patient with severe tooth wear resulted from bruxism. 62 year old patient presented with severe wear of anterior teeth (grade 3 Smith \& Knight index), loss of posterior support, and reduced occlusal vertical dimension (OVD).

The aim of the treatment was to increase the OVD in a reversible manner. Comprehensive clinical assessments were carried out using articulated study models in retruded position to construct diagnostic wax-up prior to the start of treatment.

Indirect composite build up was used to increase the occlusal dimension by $4 \mathrm{~mm}$. This was well tolerated by the patient as he had an original freeway space of $8 \mathrm{~mm}$. Severely worn upper anterior retained roots were root canal treated to maintain alveolar bone and preserve the proprioeptive capacity of periodontium. They were also fitted with gold copings to improve the stability of an overdenture. Upper (over-denture) and lower partial cobalt chromium dentures were constructed in the newly organised intercuspal position.

This treatment was successful in restoring the dentitions' form and function. The patient was reviewed in three monthly basis in which topical fluoride varnish was applied. He was also prescribed 5000ppm of sodium 
flouride toothpaste. Clinical photographs and study models were used to monitor the patient's tooth wear.

This case was complex and presented with many challenges. However, through continual discussions with the patient and the technicians, a comprehensive, sequential treatment plan, with alternative treatment options was tailored to his needs.

\section{CC43}

Pulp vitality preservation in traumatic avulsion of maxillary central incisor: case report with 2-year follow-up

Claudio Ghiberto, Riccardo Tempesta, Elio Berutti, Nicola Scotti University of Turin, Department of Surgical Sciences, Dental School, Turin, Piedmont, Italy

Objectives: This clinical case shows the clinical procedure and 2-year result for the resolution of a severe dental trauma.

Methods: The patient, a 21-years-old female, was visited in the Department of Operative Dentistry of Turin after a dental trauma, which occurred $20 \mathrm{~h}$ before. The patient showed a partial avulsion ( $6 \mathrm{~mm}$ out of alveolar bone) of 1.1 and coronal fractures on 1.1 and 2.1. After clinical and radiographic exams, which showed any bone fractures, 1.1 was immediately replaced in the correct position and splinted with glass fibres (GrandTec, Voco) to adjacent teeth. Teeth vitality was evaluated with thermal and electric pulp tests every 7 days for the following weeks. After 2 months teeth were still vital, thus splint was removed and tooth mobility was absent. Then, 1.1 and 2.1 fourth-class composite restorations were performed as follow: field isolation through rubber dam; enamel margin bevelling and finishing; etch-and-rinse adhesive system application; execution of direct restorations with Asteria (Tokuyama) in an incremental layering technique with the aid of buccal and oral silicon guides; finishing and polishing with finegrit burs and silicon points. Final radiographs were taken and follow-up visits were planned.

Results: 6, 12, 18 and 24 months follow-up showed teeth vitality and stability maintenance, soft tissue healing and perfect composite integration.

Conclusions: Immediate tooth repositioning and stable splint are key factors in the treatment of dental traumas, above all when severe damage such as avulsion (partial of complete) happens. Further follow-up are necessary to verify treatment behavior overtime.

\section{$\mathrm{CC} 44$}

Diastema closure treatment with direct resin composite restoration: clinical reports

Solen Gunal, Zeynep Batu

Department of Restorative Dentistry, Istanbul Aydin University, Istanbul, Turkey

Objective: Shape, position, size irregularities and gaps between anterior teeth are major aesthetic problems for patients. Adhesive systems enables clinicians to recontour teeth minimally or non-invasively with the aid of direct composite resin buildups to form a better smile. The aim of this study was to evaluate the clinical performans of direct composite restorations in diastema closure treatment at 6 months follow up.

Methods: A total of 82 composite restorations of 22 patients applied for diastema closure included in this study. All teeth were restored by one operator with Filtek Z250 universal restorative material (3M ESPE, USA) and dentin bonding system Clearfil $\mathrm{S}^{3}$ (Kuraray, Japan). All of the restorations were then polished with Sof-Lex finishing and polishing systems (3M ESPE, USA) from coarse to fine grits. The patients were recalled after 6 months and the restorations were evaluated using the modified USPHS criteria for retention, color match, marginal discolorization, marginal adaptation, surface texture and anatomic form.

Results: At the 6 months follow up all restorations were in situ, no complete loss was recorded and most of the restorations displayed excellent or good quality.

Conclusion: Direct composite restoration is an effective treatment when minimally invasive tooth preparation, economical and aesthetical treatment is required for closing diastemata.

\section{$\mathrm{CC45}$}

Direct composite veneers on severely eroded anterior teeth: a case report with 1year follow-up

Alessandro Pezzana

University of Turin, Turin, Piedmont, Italy

Objectives: This report deals with one case of direct composite veneers on anterior teeth with severe acid erosion. The aim of this case report is to understand clinical possibilities of extended

Methods: The patient was a 32-years-old female who presented maxillary anterior teeth with erosion of the whole buccal enamel. The patient wishes were to smile again and to reduce sensitivity to thermal stimulus. She previously visited several dentists, but she cannot afford expensive treatments because of low-economic conditions. The patient was then treated in the Operative Dentistry Department with direct composites veneers. At the first appointment, impressions were taken and a wax-up of the ideal teeth shape was performed, thus the dentist could obtain several silicon guides that are needful during composite layering. Additive restorations were then performed following a standardized clinical procedure: field isolation through rubber dam; teeth cleaning with aluminium oxide sandblasting; etch-and-rinse adhesive system application; direct additive restorations were performed using Clearfil-ES2 in an incremental layering technique with the help of buccal and oral silicon guides. Finishing and polishing procedures were then performed with fine-grit burs and silicon points. Final rx were taken and follow-up visits were planned.

Results: 7 days and 12 months follow-up showed perfect aesthetic and functional results and complete patient satisfaction.

Conclusions: Direct additive restoration can be a conservative and lowcost choice for direct composite veneers. With this technique an aesthetic smile improvement and sensitivity disappearance were obtained. Further follow-up are necessary in order to understand treatment behavior overtime. 


\section{$\mathrm{CC45}$}

\section{Neurorehabilitation with Low-Level Laser Therapy after retromolar ridge bone graft: A case report}

Despoina Chatzistavrianou, Lochana Nanayakkara

Barts Health NHS Trust, The Royal London Hospital, Turner street, Whitechapel, London, E1 1BB, UK

A wide variety of dental procedures can result to inferior alveolar nerve injury. Nerve impairment clinically is reflected as total loss of function, mild and transitory reduction of the tactile and thermal acuity or neurogenic disturbance. Although, more rapid recovery is noticed the first six months post-injury, late recovery may occur.

This case report presents a patient with numbness in the area of the inferior alveolar nerve distribution after a bone graft procedure from the left retromolar ridge that partly resolved with the application of low-level laser therapy. Patient was treated with low level laser therapy (3 Watt for 1 $\mathrm{min}$ ) in the left chin/lip, left mandible, left zygoma and left buccal sulcus once a week in 8 sessions. Subjective and objective evaluations were completed before and after the laser treatment and confirmed improvement in the sensation of the left site after the laser therapy.

Prevention of nerve damage in oral and maxillofacial interventions is the best treatment. Low level laser therapy can offer alternative treatment possibilities by improving treatment outcomes and reducing associated complications in a wide range of oral and maxillofacial interventions. Although, there is paucity of evidence on the efficiency of low level laser therapy in neurorehabilitation of inferior alveolar nerve, patients reported improvement in sensory function after the application laser treatment. Low level laser treatment is a viable treatment modality in case of paraesthesia and nerve damage taking into consideration its non-invasive nature.

\section{Dental Education (DE)}

\section{DE01}

\section{Bibliometric study of articles about DMF indices and ICDAS pub- lished on pubmed}

Adjaratou Wakha Aidara ${ }^{1}$, Christian Mesenge ${ }^{2}$, Denis Bourgeois ${ }^{3}$ ${ }^{T}$ Cheikh Anta Diop University, Dakar, Senegal, ${ }^{2}$ Université du Québec en Abitibi-Témiscamingue, Québec, Canada, ${ }^{3}$ Université Claude Bernard Lyon 1, Lyon, France

Background: Bibliometrics is an important tool in decision-making's of research policy. The evolution of clinical and epidemiological concepts has led to a new paradigm in cariology. However, few bibliometric studies are conducted on epidemiological indices used on Cariology. This transverse study aims to identify and to make a comparative bibliometric analysis of articles dealing with the two indexes of caries mainly used during the last ten years.

Methods: the methodology consisted in a bibliometric research of articles focused on DMF and ICDAS indices published on PubMed without limitation of dates and a bibliometric analysis of the results of bibliometric research.

Results: The results showed that few bibliometric studies (4 items) dealing with cariology were published. Two thousand and fifty- five (2055) articles on DMF and 91 articles on ICDAS were identified in PubMed. Thirty-six years separates the first publications on DMF and those on ICDAS. For DMF, the publication ratio was 6 articles by newspaper and 46.7 articles per year while for ICDAS it was 2.8 articles per newspaper and 11.4 articles per year. The bibliometric analysis revealed that the authors published the same types of articles for the DMF and the ICDAS. Most of the published articles were comparative studies $(41.6 \%)$, clinical trials $(13.8 \%)$, validation studies $(10.6 \%)$ and randomized clinical trials $(10.2 \%)$.

Conclusion: The results showed the need for further study by exploiting other types of bibliometric data to build a comprehensive database that will help for clinical and epidemiological decision-making based on the evidence in the field of Cariology.

\section{DE02}

\section{Effect of Education on Dental Students'Attitudes on Caries Prevention}

Hande Sar Sancakli ${ }^{1}$, Lezize Sebnem Turkun ${ }^{2}$, Yusuf Ziya Bayındır ${ }^{3}$, Cemal Yesilyurt $^{4}$, Gamze Yildirim ${ }^{2}$, Merve Iscan ${ }^{3}$, Tugba Serin Kalay ${ }^{4}$, Sevda Ozel Yildiz ${ }^{5}$, Taner Yucel ${ }^{1}$, Esra Yildiz ${ }^{1}$

${ }^{1}$ Istanbul University Faculty of Dentistry Department Of Restorative Dentistry, Istanbul, Turkey, ${ }^{2}$ Ege University Faculty of Dentistry, Department Of Restorative Dentistry, Izmir, Turkey, ${ }^{3}$ Ataturk University Faculty of Dentistry, Department Of Restorative Dentistry, Erzurum, Turkey, ${ }^{4}$ Karadeniz Technical University Faculty of Dentistry, Department Of Restorative Dentistry, Trabzon, Turkey, ${ }^{5}$ Istanbul University Faculty of Medicine, Department of Isthatistics, Istanbul, Turkey

Aim: Dental caries remains as the most common chronic disease which can be prevented to decrease the prevalence and severity. The contemporary dentistry approach requires caries risk assessment and prevention which is already included in the undergraduate curriculum. The aim of this multi-centered questionnaire was to assess the effect of caries prophylaxis education within the dental curriculum on the knowledge, attitudes, and intended behaviors in caries prevention of dental students from 4 dental schools located in different regions of Turkey.

Materials and methods: 281 fifth-year students were included in a thirty-seven-itemed written opinion and knowledge regarding a caries prophylaxis based questionnaire. Data were gathered and the total frequency was determined.

Results: $95 \%$ of the students reported that caries can be inhibited by prophylactic measures performed at the 6-12 months recalls. $96 \%$ evaluated fissure sealants applied on the first and second molars as an effective method to inhibit caries whereas $60 \%$ would prefer fluoride gel as first choice. $86 \%$ of the students would recommend sugar-free chewing gums with an increasing rate and $85 \%$ considered that snack meals increases caries risk. $43 \%$ of the students reported that they would use caries diagnostic tests for the high-caries risk patients while only $14 \%$ would use them for all. $84 \%$ would recommend routine check-ups while $78 \%$ would apply fluoride gel as a primer preventive measure.

Conclusion: The effect of the education regarding the caries prevention awareness seems to raise and increase the attitudes of dental students toward implication of preventive techniques in their daily practice. 


\section{DE03}

The application of haptic simulation in manual dexterity performance in first dental students.

Anuradha Polster, Michael Francis Burrow, Matthew Hopcraft The University of Melbourne, Victoria, Australia

The aim of this study was to evaluate manual dexterity performance and development of 70 first year Doctor of Dental Surgery students at the Melbourne Dental School using MOOG Simodont haptic dental trainers in association with traditional preclinical training on phantom head manikins.

Students performed a series of gradually more complex 3D tasks that simulated cutting shapes encountered in Operative Dentistry on the haptic dental trainer. The Simodont is able to generate reports on a range of parameters, including the number of attempts for each task, the total 'drilling' time, the percentage of the $3 \mathrm{D}$ target shape removed, and the percentage removed beyond the leeway space (greater than the desired shape) around the task. The parameters generated from the reports were then compared with assessment scores from the phantom head exercises later in the first year. Phantom head tasks included various cavity preparations in plastic teeth. The project was approved by the University Humans ethics committee; signed informed consent was obtained from participants.

It was shown that as students progressed on the more complex Simodont tasks their manual dexterity improved. A few students showed continual difficulty in completing the complex tasks even after extended practice. It was also demonstrated that level of performance on the 3D haptic tasks was a good predictor of later performance in phantom head tests.

It was concluded that the SImodont may be a useful tool for early identification of students who may encounter difficulties in developing dexterity skills.

\section{DE04}

CAFS - Enhancing teacher feedback and learner reflection in clinical dental education

Upen Patel, James Kelly, David Attrill, Deborah White, Giles Perryer School of Dentistry, University of Birmingham, Birmingham, UK

Educating clinical dental students in a busy multi-chair clinic is challenging, and time pressures can often result in clinical educators becoming mere supervisors. CAFS (Clinical Assessment and Feedback System) provides a tablet-based transparent quality assurance tool that ensures educators provide learners with (recorded) constructive feedback for each clinical session, and that learners consider the feedback received, and record their reflections. A typical clinical learning episode using CAFS would have the learner record the procedures they had undertaken. The patient then has the opportunity to provide confidential feedback based on their experiences with the dental student. The educator records performance grades (assessment) and feedback into the system. Finally, the learner is expected to record their reflections and learning points from their experience of treating the patient. This final step allows the learner to achieve a higher level of learning.
Having educator feedback stored electronically and being available online allows the learner to reflect on the complete learning process when they wish rather than at the time of the learning episode. The learner is also able to check their ranking within the cohort, putting their clinical progress into perspective. Holding the information in this way facilitates educator calibration and ensures transparency of information between all educators and learners. This system has shown to increase the quality of feedback by educators. Learners have also demonstrated an increase in reflective - higher-level - learning. We believe this system significantly enhances clinical dental education and is the future for effective learning in a clinical environment.

\section{DE05}

The CAD/CAM unit in Paris Descartes University: for and by students

Anne-Margaux Collignon ${ }^{1,2}$, Claudine Wulfman ${ }^{1,3}$, Boris Jakubowicz ${ }^{1,4}$, Mathieu Derbanne ${ }^{1}$, Bruno Zamansky ${ }^{1}$, Nicolas Lebon ${ }^{1,5}$, Stephane Le Goff $^{1,4}$, Aurelie Dubroca ${ }^{1,3}$, Laurent Tapie ${ }^{1,5}$, Jean-Pierre Attal ${ }^{1,4}$

${ }^{1}$ Paris Descartes University, Biomaterials laboratory URB2i-EA 4462, Platinum, Paris, France, ${ }^{2}$ Paris Descartes University, Department of Conservative Dentistry, Paris, France, ${ }^{3}$ Paris Descartes University, Department of Prosthodontics, Paris, France, ${ }^{4}$ Paris Descartes University, Department of Biomaterials, Paris, France, ${ }^{5}$ Paris Descartes University, Department of Engineering Research, Paris, France

Objective: To include CAD/CAM technologies in oral rehabilitation teaching.

Three difficulties arise when bringing and applying the theoretical knowledge in clinical training:

- To offer a standard teaching to all students,

- In 4 dental units located in different hospitals,

- Under a high level supervision.

Materials and method: A dedicated room is equipped with three different $\mathrm{CAD} / \mathrm{CAM}$ systems and a $3 \mathrm{D}$ printer, as well as characterization and polishing kits (ceramic, composite and furnace). In clinic, with his teacher, the student indicates the partial restoration and performs preparation and impression (conventional or optical). Design and manufacturing are realized at the faculty under the supervision of a multidisciplinary team, including biomaterials, prosthodontics and conservative dentistry teachers, prosthodontics technicians, engineering specialists and researchers.

Results: In this privileged dialogue about a patient, the student discovers the unit scientific interest, as all clinical relevant information about patients, clinical situation and restoration features is recorded for a long-term cohort study. Teachers note a better comprehension of preparation needs and tissue preservation while the student manages the whole process of partial denture restoration. His understanding widens when confronted to clinical situation, cast model and $3 \mathrm{D}$ reconstruction.

Discussion and conclusion: $\mathrm{CAD} / \mathrm{CAM}$ is a key teaching in 21 st century dental curriculum. These new technologies confront us to a new educational challenge. Because of the limited background available, a multidisciplinary team is necessary to explore the opportunities they open as well 
as their limitations. From this point of view, collaboration with researchers is essential.

\section{DE06}

\section{Comparing the Effectiveness of Dental Health Education Program} Using Digital Aids and Conventional Methods

Kundabala Mala

Manipal College of Dental Sciences, Mangalore, Mangalore, India

Introduction: Health education is very important tool for health promotion, helps to improve the health of populations, thus promote health capital. Access to appropriate education tools, engaging people in effective health education practice, reaching people of remote and rural areas, time and cost involved in health education and effective and timely support from government are some of the challenges that exist. Advances in technology such as computers and images in a multimedia presentation have made the messages to be delivered more comprehensively to patients to help them understand the available treatment options.

Objectives: the present study was undertaken to evaluate and to compare the effectiveness of Dental Health Education program using digital aids with conventional methods in improving oral health knowledge, practices and oral health status of dental patients.

Methodology: The study utilized "Activated Health Education Model". A total no. of 150 individuals divided randomly into 2 groups of 75 each for conventional and digital technique respectively. Pre-education questionnaire was collected to evaluate oral health knowledge, awareness and attitude from all the samples. Pre-education plaque index, DMFT and gingival scores were recorded. Education was conducted by digital and conventional methods. 3 months post education mean scores with standard deviation were tabulated for both conventional and digital education techniques. Statistical analysis of Variance was done using one way Anova and post Hoc test.

Conclusion: There is no difference between digital aids and conventional educational methods but dental education has a definite role in improving patient knowledge, awareness regarding oral health.

\section{DE07}

Dental pre-clinical education with haptic-based virtual reality: state of art and challenges

Minh Tran Bui ${ }^{1,2}$, Céline Gaucher ${ }^{1,2}$, Jean-Jacques Lasfargues ${ }^{1,3}$ ${ }^{T}$ Conservative dentistry and Endodontics department of Paris Descartes dental school, Montrouge, France, ${ }^{2}$ Albert Chenevier Hospital, GHHM, AP-HP, Creteil, France, ${ }^{3}$ Bretonneau hospital, HUPNVS, AP-HP, Paris, France

Introduction: Virtual reality simulation (VRS) has already proved its effectiveness in high reliability organisations (aviation, surgery...). It is now reaching the field of dental education, very sensitive to the integration of gestural dexterity with medical skills and knowledge.

Objectives: This study aims to explore the main existing simulators available on the market and assess the potential impacts of haptic-based VRS on dental student formation.
Materials and methods: A literature review on haptic-based VRS was performed and a comparison of the main commercial systems (VirTeaSy, HRV ${ }^{\mathbb{B}}$; Simodont, Moog ${ }^{\circledR}$ and Voxel-Man Denta, Voxel-Man ${ }^{\circledR}$ ) was elaborated after dataset analysis and experimental testing. In addition, an assay with students and teachers was performed on the VirTeaSy simulator at the Paris Descartes dental school.

Results: Literature analysis as well as simulator comparison have led to the conclusion that haptic-based VRS would be an interesting complementary system to conventional trainings. Relevance of the simulators is demonstrated in terms of construct and content validities. While satisfactory, face validities and ergonomics have to be improved. Live tests at the Paris Descartes dental school confirm these data and point to the high degree of adaptability of novice face to simulators.

Conclusion: Haptic-based VRS systems are promising tools for dentist education. They allow progressive skill acquisition together with self-assessment, and interactive follow up of the learning curve for teachers. For a better integration, simulators contents should be thought in-depth within university communities and further research needs to be conducted.

\section{DE08}

Qualitative Analysis of Dental Practitioners' Perceptions of a Part Time Master's in Restorative Dentistry

Peter Fine, Albert Leung, Chris Louca

UCL Eastaman Dental Institute, London, UK

Objective: This study explores the perceptions of General Dental Practitioners (GDPs) following the completion of a five year, part time MSc in Restorative Dental Practice (RDP). It also explores potential new pedagogy for programme development. Qualitative data were collected. The learning experience and perceived impact of new knowledge and skills on general dental practice were explored.

Method: Qualitative data from the GDP participants were collected via questionnaires and individual interviews. The questionnaires requested responses to open ended questions on overall perceptions of the programme, perceived impact of the programme on clinical practice and future career aspirations. The interviews (in person or by telephone) were semi structured and complimented the questionnaire.

Results: 18 questionnaires were distributed. 94\% $(n=17)$ were returned and $61 \%(\mathrm{n}=11)$ of the participants were interviewed. Analysis of the qualitative data revealed: i) achievement of an overall satisfactory learning experience ii) a significant improvement in confidence iii) positive changes to clinical practice iv) work related restrictions in implementing newly acquired knowledge and skills and v) a tendency to use personal development plans for future studies. Criticisms included a lack of feedback from the teachers and inconsistences with student supervision.

Conclusion: Following the MSc, GDP participants perceived the programme had a positive impact on clinical practice and a distinct improvement in their confidence to undertake restorative dental treatment. An increase in treatment uptake by the patients and a perceived improvement in employment opportunities were also noted. In addition teachers on such PG programmes should be adequately trained and appropriately mentored. 


\section{Endodontics \& Pulp Biology (EP)}

\section{EP01}

Clinical outcomes of non-surgical root canal treatment of mandibular second molar with $\mathrm{C}$-shaped canals

Hye-Ra Ahn, Young-Mi Moon, Min-Seock Seo

Wonkwang university daejeon dental hospital, Daejeon, Chungcheongnam-do, Republic of Korea

The aims are to evaluate the clinical outcomes of non-surgical root canal treatment of mandibular second molar with the comparison between $\mathrm{C}$ shaped and non-C-shaped canals in terms of periradicular healing and tooth survival, and to investigate their prognostic factors.

Total 109 teeth treated by non-surgical root canal treatment were analyzed retrospectively. The clinical outcomes of non-surgical root canal treatment were determined by clinical and radiographic evaluation. Periradicular healing and tooth survival estimates were compared using Kaplan-Meir analysis. The contributions of a patient's age and gender, number of canal, canal type, tooth location, preoperative diagnosis, and existence of crack were investigated using the multivariate Cox proportional hazard model.

The mean follow-up period was 24.2 months. The overall survival rate was $57.8 \%$ and the survival rate of teeth with $\mathrm{C}$-shaped canals was $60.6 \%$. The survival rates were $41.7 \%$ for cracked teeth and $51.8 \%$ for teeth with apical periodontitis. Amongst the variables tested, existence of crack, and preoperative periradicular status significantly affected the survival rate of a tooth. Other prognostic variables such as age, gender, number of canal, and tooth location did not affect the survival of mandibular second molar with C-shaped canals after non-surgical root canal treatment.

In conclusion, mandibular second molar with C-shaped canals, which has been vaguely considered hard to be treated, did not suggest necessarily the higher failure of root canal treatment than non-C-shaped canals in this study. Preoperative existence of crack and preoperative diagnosis were the most influential factors of treatment failure.

\section{EP02}

In Vitro Assessment of Force Generated by Different Novel NickelTitanium Rotary Systems

Anwer Eshaq, Salman Alsalman, Federico Foschi, Francesco Mannocci King's College Of London, London, UK

Aim: The aim of the present study was to compare in-vitro the force necessary to reach the apical portion of the natural root canals in extracted human teeth of the new PTN files with other files including PTU, Revo-S and $\mathrm{RaCe} 123$ by a miniature load-testing machine.

Methodology: Eighty mesio-buccal canals of extracted human permanent maxillary first and second molars teeth were manually prepared, to the pre-determined working length, up to a size ISO $15 \mathrm{~K}$-flexofile. The teeth were randomly assigned to four groups of 20 samples each (PTN, PTU, RaCe123, and Revo-S). The teeth were mounted on one end of a miniature load-testing machine while the files/handpiece assemblies were mounted on the other. The canal preparation was undertaken at a pre-set velocity towards the apex, while the machine measured the apical force applied on the file. Maximum and average forces were calculated and statistically analysed using Kruskal-Wallis and Mann-Whitney-U test analyses of variance followed by the Bonferroni correction for multiple testing.
Results: Statistically significant differences in the forces applied by the different filing systems were noted in the experimental groups, with the Revo-S and PTN generally scoring the lowest average forces (with the concomitant highest peaks) when reaching the apical third and fourth quarters of the canal length, followed by RaCe123 and PTU.

Conclusion: The results, within the limitations of this study, indicated that the ProTaper Next and Revo-S systems required less apical force during root canal shaping than ProTaper Universal and RaCe 123.

\section{EP03}

\section{Bacterial invasion into radicular dentine}

Simone Stauffacher ${ }^{1}$, Klaus W. Neuhaus ${ }^{1}$, Adrian Lussi ${ }^{1}$, Sigrun Eick ${ }^{2}$ ${ }^{T}$ University of Bern: Department of Preventive, Restorative, and Pediatric Dentistry, Bern, Switzerland, ${ }^{2}$ University of Bern: Department of Periodontology, Bern, Switzerland

Introduction: Penetration of bacteria into radicular dentine, may lead to endodontic failure because these microorganisms are protected against chemo-mechanical treatment. Most endodontic persistent infections are not caused by only a single species.

Aim: The aim of this study was to investigate differences in penetration of root dentinal tubules by mono-cultured and by co-cultured bacteria frequently found in infected root canal.

Materials and Methods: 51 human roots were inoculated with mono-cultured Streptococcus gordonii ( $\mathrm{Sg}$ ), S. sanguinis (Ss), and five anaerobes as well as with anaerobes co-cultured with a streptococcal species for 8 weeks. Thereafter samples from the inner, middle and outer part of the root dentine of lengthwise broken teeth $(n=5)$ were cultivated. In addition, scanning electron microscopy (SEM) photographs were taken.

Results: Single grampositive species were able to penetrate onto the middle and outer part of the root dentine. Fusobacterium nucleatum ( $F n$ ) was not found in any of the dentine pieces, Prevotella intermedia (Pi) and Porphyromonas gingivalis in the inner and middle parts.

Except for co-culture with $P i$, streptococci were always found more often in middle and outer parts in co-cultures when compared with single infection. In co-cultures with streptococci $A$. viscosus was found in 9/10 samples in the outer parts, whereas $P i$ was not detectable in dentine. Coculture with $S s$ enabled $F n$ to invade dentine; SEM photographs showed a swollen shape of $F n$.

Conclusions: Oral streptococci promote or inhibit invasion of anaerobes in radicular dentine.

\section{EP04}

Representative Survey on the Reprocessing of Endodontic Instruments in Germany

David Sonntag, Evelyn Martin, W.H.-M. Raab

Department of Operative and Preventive Dentistry, Periodontology and Endodontology, Düsseldorf, Germany 
Aim The aim of the study was to review the implementation of hygiene guidelines for the reprocessing of endodontic instruments in a representative survey and to determine the time needed for the procedure.

Methodology A questionnaire with nine items was sent to 4000 German dentists. In addition to obtaining personal information, the procedure and total time required for the reprocessing of instruments were calculated to determine whether the requirements of the German Federal Ministry of Health were complied with. The data were analyzed statistically using the Chi-square test and Cramer's $\mathrm{V}$ as a measurement of effect size.

Results The response rate was 29.4\%, 1118 (27.95\%) questionnaires could be included in the evaluation. With effect sizes between 0.12 and 0.21 , the survey could be classified as statistically representative of German dentists in terms of age and gender. $68.3 \%$ of respondents adhered the guidelines either not fully or not at all $(P<0.001)$. There were no significant differences in adherence to guidelines between younger $(<45$ years) and older ( $>44$ years) dentists $(P=0.179)$ or between women and men $(P=0.194)$. The reported time for cleaning a patient-related instrument set was more than $9 \mathrm{~min}$ for $68.1 \%$ of respondents, not including the time needed for sterilization; $5 \%$ of respondents treated files as single-use instruments.

Conclusions The guidelines of the German Federal Ministry of Health for cleaning, disinfecting and sterilizing endodontic instruments are widely disregarded in Germany. The designation and use of endodontic instruments as single-use instruments should be considered.

\section{EP05}

Cyclic fatigue resistance of conventional and new generation nickeltitanium rotary instruments. an in vitro comparison.

Celia Ruiz-Sánchez, Vicente Faus-Matoses, Teresa Alegre-Domingo, Vicente J. Faus-Llácer

Valencia University. Master in Restorative Dentistry and Endodontics, Valencia, Valencia, Spain

Objective: The purpose of this study was to compare the cyclic fatigue resistance (FC) of 3 nickel-titanium (NiTi) endodontic instrument, ProTaper Next (PTN; Dentsply Maillefer, Ballaigues, Switzerland), Profile Vortex Blue (PVB; Dentsply Tulsa Dental, Tulsa, OK, USA) and ProTaper Universal (PTU; Dentsply Maillefer, Ballaigues, Switzerland).

Methods: Cyclic fatigue test was conducted by operating instruments from ProTaper Next X2, Profile Vortex Blue 25.06 and ProTaper F2. A total of 198 instruments were rotated in 2 simulated stainless steel curved canals with different angles of curvature $\left(45^{\circ}\right.$ and $\left.60^{\circ}\right)$ and $5-\mathrm{mm}$ radius of curvature. The number of cycles to fracture (NCF) was calculated. Data were compared by using 2-way analysis of variance $(\mathrm{P}<0.05)$.

Results: PVB showed better resistance to cyclic fatigue in both curved canals than PTN and PTU. The lowest resistance to cyclic fatigue canals were obtained by PTU.

Conclusions: From the tested rotary files, PVB was the most resistant to fatigue failure; and PTN showed higher number of cycles to failure than PTU.
EP06

C-shaped root canals of mandibular second molars: an in vivo conebeam computed tomography study

Yemi Kim

Department of Conservative Dentistry, Ewha Womans University, Seoul, Republic of Korea

Objective: To investigate the anatomical features of C-shaped root canal system in mandibular second molars in a Korean population by using cone-beam computed tomography (CBCT).

Methods: A total of 960 subjects with bilateral mandibular second molars were enrolled. The prevalence of $\mathrm{C}$-shaped root canals and their anatomical features were determined. Root canal configurations at four different levels were categorized based on modified Melton's classification. Differences in the prevalence of C-shaped root canal systems between sex, age, and tooth position (right or left side) were compared using the Chi-squared test.

Results: Of the 1,920 mandibular second molars, the prevalence of C-shaped roots was $40.10 \%$. C-shaped roots in combination with additional mesiolingual or distolingual roots were found in $0.73 \%$ of molars. Of the C-shaped roots, the most common configuration types were Melton's type I (66.36\%) in the coronal region and Melton's type III $(55.58 \%)$ in the apical region. The prevalence of C-shaped roots was higher in females $(46.92 \%)$ than in males $(32.09 \%)(P<0.001)$ and did not differ with age $(P=0.497)$ or tooth position $(P=0.514)$. Most $(82.34 \%) \mathrm{C}$-shaped canals were symmetrical $(P<0.001)$.

Conclusions: A high prevalence of C-shaped canals were observed in the mandibular second molars of the Korean population. The configuration of $\mathrm{C}$-shaped canals varied along the length of the root.

This research was supported by the National Research Foundation (NRF-2012R1A1A1012913 and NRF-2012M3A9B6055379) of South Korea.

\section{EP07}

Sealing ability of 2 root-end filling materials: a comparative in-vitro study

Blanca Faci-Martín $^{1}$, Vicente Faus-Matoses ${ }^{2}$, Teresa Alegre-Domingo ${ }^{3}$, Vicente J. Faus-Llácer ${ }^{4}$

${ }^{1}$ Valencia University. Master Student in Restorative Dentistry and Endodontics, Valencia, Valencia, Spain, ${ }^{2}$ Valencia University. Associate Professor of Master in Restorative Dentistry and Endodonics., Valencia, Valencia, Spain, ${ }^{3}$ Valencia University. Associate Professor of Master in Restorative Dentistry and Endodonics., Valencia, Valencia, Spain, ${ }^{4-}$ Valencia University. Professor and Director of the Master in Restorative Dentistry and Endodonics., Valencia, Valencia, Spain

Introduction: Periradicular surgery involves the root-end cavity preparation and its filling. MTA is considered the gold-standard retrograde filling material.

Recently, a bioceramic cement which is called Biodentine (Septodont, Saint Maur des Fossés, France), with similar and improved properties compared to MTA, has been introduced. 
The aim of this study is to evaluate the apical leakage of MTA (Dentsply Maillefer, Ballaigues, Switzerland) compared to Biodentine when used as retrograde filling materials.

Material And Methods: 70 intact, single-rooted with single-canals teeth were transversally sectioned bellow the cementum-enamel junction

Canals were shaped with Protaper Unisversal System (Dentsply Maillefer, Ballaigues, Switzerland) up to size F2.

Specymens were obturated with warm gutta-percha and resin sealer. Retrograde cavities were prepared.

Teeth were randomly divided into two groups $(\mathrm{n}=33$ ) according to the retrograde filling material used: MTA and Biodentine. Two groups $(\mathrm{n}=$ 10) were used as positive and negative control.

Specymens were submerged in Rhodamine solution and were cut longitudinally into two halves. Each halph was examined under confocal laser microscope. The area of greater dye penetration was measured in $\mathrm{mm}$ to establish the marginal apical leakage of each specymen.

Chi-squared Test was used to statically analyze the results. T-test was used to compare independent samples. Significance level was set at $5 \%$.

Results: MTA showed statistically lower apical leakage compared to Biodentene when used as retrofilling material. However, differences between both groups were not statistically significant.

Conclusion: Although no group showed a perfect sealing of rootend cavities, both MTA and Biodentine, could be used as retrofilling materials.

\section{EP08}

Photodynamic inactivation of intracanal bacteria by light-activation through dental hard and surrounding tissue in vitro

$\underline{\text { Fabian Cieplik }}^{1}$, Andreas Pummer ${ }^{1}$, Johannes Regensburger ${ }^{2}$, Tim Maisch $^{2}$, Karl-Anton Hiller ${ }^{1}$, Gottfried Schmalz ${ }^{1,3}$, Wolfgang Buchalla ${ }^{1}$

${ }^{1}$ Department of Conservative Dentistry and Periodontology, University Medical Center Regensburg, Regensburg, Germany, ${ }^{2}$ Department of Dermatology, University Medical Center Regensburg, Regensburg, Germany, ${ }^{3}$ Department of Preventive, Restorative and Pediatric Dentistry, School of Dental Medicine, University of Bern, Bern, Switzerland

Photodynamic inactivation of bacteria (PIB) may be a supportive antimicrobial tool for use in endodontics. Aim of this in vitro study was to evaluate the ability of photosensitizers (PS) to be light-activated through dental hard and surrounding tissues, in order to avoid the use of intracanal optical fibres for application of PIB in endodontics.

A tooth-model was built from a human premolar and two molars arranged in a mixture of Paladur-resin simulating the optical properties of a porcine jaw. The distal root canal of the first molar was expanded for inserting a glass-pipette containing the PS and stationary-phase Enterococcus faecalis $(E F$; ATCC-29212; OD = 0.1). Blue-light absorbing TMPyP and red-light absorbing Methylene Blue (MB) were used as PS in concentrations of $10 \mu \mathrm{M}$. TMPyP was irradiated for $120 \mathrm{~s}$ with BlueV (Waldmann; $\lambda=400-460 \mathrm{~nm} ; 20 \mathrm{~mW} / \mathrm{cm}^{2}$ ) and $\mathrm{MB}$ for $120 \mathrm{~s}$ with PDT1200L (Waldmann; $\lambda=570-680 \mathrm{~nm} ; 37.8 \mathrm{~mW} / \mathrm{cm}^{2}$ ). Irradiation parameters ensured identical numbers of photons absorbed by each PS. Three set-ups were performed: irradiating the glass-pipette only (I), the glass-pipette placed in the single tooth without (II) and with (III) simulated surrounding tissues. Colony forming units (CFU) were evaluated ( $\mathrm{n}$ $\geq 6$; controls: untreated, PS only, light only)

$\mathrm{PIB}$ with both PS led to reduction rates of at least $5 \log _{10}$ steps of $E F$-CFU for each set-up used, indicating that blue and red light was able to penetrate simulated dental hard and surrounding tissues to activate both PS. Consequently, using TMPyP or MB it might be possible to light-activate intracanal PS from outside the tooth through dental hard and surrounding tissues.
EP09

Push-out bond strength of fiber post to root dentin using three different resin cements

Lourdes Garcia-Gimenez, Vicente Faus-Matoses, Teresa Alegre-Domingo, Vicente Faus-Llacer

University of Valencia, Valecia, Spain

Objectives: The aim of this in vitro study was to evaluate the push-out bond strength of three different cements to root dentin in three different thirds of the root canal.

Methods: Ninety human intact teeth single rooted lower incisors were transversally sectioned $1 \mathrm{~mm}$ above the cementum-enamel junction. Canals were prepared with Protaper Universal system (Dentsply Maillefer, Ballaigues, Switzerland) and treated with guttapercha and Top Seal (Dentsply Maillefer, Ballaigues, Switzerland) sealer. Prepared teeth were randomly assigned to one of three groups $(\mathrm{n}=30)$ according to the cement used for post cementation: Core.X Flow (Dentsply DeTrey, Konstanz, Germany); SureFil ${ }^{\mathbb{R}} \mathrm{SDR}^{\mathrm{TM}}$ (Dentsply,G); SmartCem2 (Dentsply, G). After cementation of glass-fiber post, all roots were stored at $100 \%$ humidity until testing. For push-out test, 2.5-mm thick slices were produced. Push-out bond strength was assessed at three different levels: cervical, middle and apical using a universal testing machine at a crosshead speed of $0.5 \mathrm{~mm} / \mathrm{minute}$. KolmogorovSmirnov test was used to evaluate the distribution and homogeneity of the data. The values (MPa) were analysed by two-way analysis of variance and Tukey post hoc tests at a significance level of $5 \%$.

Results: No significant difference in bond strength was showed between the groups. Core.X Flow and SureFil ${ }^{\mathbb{R}} \mathrm{SDR}^{\mathrm{TM}}$ groups demonstrated statistically lower bonding values in the apical region when compared with the middle and cervical region. SmartCem2 had no significant differences along the root thirds.

Conclusion: The resin cements used exhibited similar bond strengths. The lowest bond strength was observed in the apical third for the Core.X Flow and SureFil ${ }^{\mathbb{R}} \mathrm{SDR}^{\mathrm{TM}}$ groups.

\section{EP10}

Micro-CT analysis of root canal space of teeth restored with fiber glass posts

Renata Chalas ${ }^{1}$, Marta Jurczykowska ${ }^{1}$, Karol Szlazak ${ }^{2}$, Jakub Jaroszewicz ${ }^{2}$, Wojciech Swieszkowski ${ }^{2}$, Krzysztof Jan Kurzydlowski ${ }^{2}$

${ }^{1}$ Department of Conservative Dentistry and Endodontics, Medical University of Lublin, Lublin, Poland, ${ }^{2}$ Faculty of Materials Science and Engineering, Warsaw University of Technology, Warsaw, Poland

Intracanal posts are commonly used in endodontics, nowadays. However, correct obturation and reconstruction of the root canal space is crucial for the long-term prognosis of a tooth. Micro-computed tomography may provide useful and detailed insights into this procedure. The aim of this study was to evaluate the area/volume of endodontically treated teeth that has been restored with fiber glass posts by means of micro-CT.

Material and methods: Single-rooted extracted teeth after endodontic treatment were prepared and restored with XCore\&Post System 
(Dentsply Maillefer) and rebuild with a CoreX-flow. All teeth were examined with a SkyScan 1172 micro-CT (Bruker) with a voxel size of 11.0 $\mu \mathrm{m}$. The 3D reconstructions were performed using NRecon software, and slices were analyzed using CTAn. DataViewer and CTVol were used to show the microstructure of restored teeth.

Results: The three-dimensional visualization (longitudinal, transverse, sagittal and volume presentation) revealed heterogeneous areas in restored root canals. Air voids were mainly detected in the coronal part of the teeth, and their diameter ranged from 300-1200 $\mu \mathrm{m}$. These pores were only found in the cement space and were not visible at the cement-dentine interface.

Conclusion: 3D investigation of the structure and the position of the fiber post and the cement in the root canal may help to ensure an accurate clinical procedure to avoid pores in the restoration, and to protect from tooth fracture.

\section{EP11}

\section{A novel luting resin composite core material}

Ahmed Subhy, Luis Rojo, Sanjukta Deb

King's College London, London, UK

Objectives: The purpose of this study was to develop novel resin composites tailored to function both as luting medium for post placement and core build up material via inclusion of a methacrylate derivative of eugenol in a bisGMA/TEGDMA resin system. The effect of inclusion of this antibacterial monomer on the physical and mechanical properties were determined and compared with a commercially core material (Clearfil $\left.{ }^{\mathrm{TM}} \mathrm{DC}\right)$.

Methods: The antibacterial monomer eugenyl methacrylate (EgMA) was synthesized and two resin composites formulated with $5 \& 10 \%$ by weight of EgMA. The base formulation consisted of bisGMATEGDMA reinforced with $65 \%$ of a hydroxyapatite/zirconia filler. The degree of monomer conversion was evaluated from FTIR spectra and depth of cure, dynamic, static mechanical properties and radiopacity determined.

Results: The degree of cure ranged from 65 to $74 \%$, which was comparable to the Clearfil ${ }^{\mathrm{TM}}$ and within acceptable standard. The depth of cure of the experimental composites ranged from 8.3 to $9.8 \mathrm{~mm}$ compared to the Clearfil $4.5 \mathrm{~mm}$. The incorporation of 5\% EgMA resulted in $114.8 \pm$ $6.8 \mathrm{MPa}$ and $231.34 \pm 18.9 \mathrm{MPa}$ average flexural and compressive strength respectively. The radiopacity of the experimental composites (equivalent to $\sim 3 \mathrm{~mm} \mathrm{Al}$ ) was superior to that of the commercial core material and sufficiently higher than dentine and enamel to allow efficient diagnosis.

Conclusion: The inclusion of EgMA as an antibacterial monomer into commonly used dental methacrylate resin provides a novel approach in imparting antibacterial properties without the inhibitory effect of phenol derivatives.

\section{EP12}

The influence of the irrigation protocol in the organic and inorganic radicular dentin composition.

Marta Baron ${ }^{1}$, Nuria Escribano ${ }^{1}$, Maria Linares ${ }^{2}$, Victoria Morales ${ }^{2}$, Maria Victoria Fuentes ${ }^{1}$, Laura Ceballos ${ }^{1}$
${ }^{1}$ Rey Juan Carlos University, Alcorcón, Madrid, Spain, ${ }^{2}$ Rey Juan Carlos University, Móstoles, Madrid, Spain

The irrigation solutions not only eliminate bacterial infection when used in root canal treatment, but they can also induce chemical and physical changes in the composition of radicular dentin.

Objective: To evaluate the changes of organic and inorganic composition of radicular dentin using different irrigation solutions.

Materials and methods: Four single root teeth were shaped with Protaper Universal system (Dentsply Maillefer). Roots were sectioned perpendicularly to the long axis. Three $500 \mu \mathrm{m}$ thick sections were obtained from each root third, and randomly distributed in different testing groups. GROUP I: NaOCl 5.25\% (1 minute) - GROUP II: $\mathrm{NaOCl}$ $5.25 \%$ (5 minutes) - GROUP III: $\mathrm{NaOCl} 5.25 \%$ (20 minutes) GROUP IV: EDTA 17\% (1 minute) - GROUP V NaOCl $5.25 \%$ (20 minutes) - EDTA 17\% (1 minute) - NaOCl 5.25\% (1 minute). Samples were analyzed by Fourier Transform Infrared spectroscopy (Excalibur 3010 FT-IR, Varian) to determine organic and inorganic components before and after irrigation solution protocol. Data were analyzed by with two-way Anova, and SNK $(\mathrm{p}<0.05)$.

Results: In the Group I, II, III, and V, the Amida III and Amida I decreased in apical third.

Conclusions: The organic component decreased in the apical third when $\mathrm{NaOCl} 5.25 \%$ was used either in combination with EDTA $17 \%$ or not.

\section{Operative Dentistry (OD)}

\section{OD01}

Effectiveness of optical illusion techniques for anterior diastema closure with one composite resin veneer.

Thomai Katsarou, Maria Antoniadou, Stratis Papazoglou Dental School of Athens, Athens, Greece

Aim: To assess the esthetic effectiveness of 5 techniques for diastema closure between the maxillary central incisors intervening only on one of them with a composite resin veneer.

Methods: An acrylic model with 6 natural maxillary anterior teeth having a $2 \mathrm{~mm}$ diastema between the central incisors was fabricated. Left central incisor \#21, was prepared for laminate veneer. Composite veneers were constructed (V0:no veneer, V1:veneer without optical illusion, V2:veneer with centralized interproximal ridges, V3:veneer with curved incisal edges, V4:veneer with grey pigment mesially/distally, V5:veneer with grey pigment on developmental lobes). Digital photos (size $\mathrm{a}: 13,2 \times 17,8 \mathrm{~cm}, \mathrm{~b}: 6,1 \times 8 \mathrm{~cm})$ of the restorations, were given for evaluation to 75 persons $(n=25$ persons per group of specialists, dental students, and patients) in standardized conditions. Results were analyzed by $\mathrm{X}^{2}$ test $(\mathrm{a}=0.05)$, Stuart-Maxuell and Bonferroni Correction test.

Results: Method had a significant influence on the assessment of both width and length. The incisor in a lower probability was 
assessed as wider in V0 $(99,4 \%, \mathrm{p}<0,001)$ and longer in V0 $(63,1 \%), \mathrm{V} 3(81,5 \%)$ and V4 $(63,8 \%)$ compared to V1. Group and gender had significant influence only on the assessment of length. Students had reduced probability to consider incisor longer $(59,6 \%)$ compared to specialists and women $(60 \%)$ compared to men.

Conclusions: V0, V3 and V4 are effective when incisor is desired to seem shorter. Patients' perception is reduced compared to that of dentists and students' compared to specialists'. Gender interferes in the perception of length.

\section{OD02}

\section{Closing the Gap: the role of virtual reality in linking preclinical and} clinical operative skills

Catherine Snelling

University of Adelaide, Adelaide, Australia

It could be argued that more than any other health practitioner, dental operators require a high level of manual dexterity at graduation. Minimum intervention dentistry has added clinical procedures such as tunnel preparations that require exquisite dexterity, and an increasing number of dentate patients in the elderly population has increased demand for skilful periodontal and endodontic care.

With this evolution of dental care in mind, fledging practitioners must be given the opportunity to nurture operative skills in an environment that allows them to develop confidence and competence before commencing clinical practice on patients. Contemporary dental educators need to design and implement a preclinical program in their courses that scaffold skill development in parallel to knowledge acquisition. Rather than students moving straight across the divide from somewhat 'static' typodonts to 'real-world' mouths, many dental programs are closing the gap with the inclusion of virtual reality technology as a strategic stage in their operative technique courses.

This paper will provide an overview of international trends in the role of virtual reality in dental education. It will then focus on the strategies that have been introduced as part of an evidence-based approach to embedding virtual reality in the School of Dentistry at the University of Adelaide, Australia. Data and outcomes from two years of implementation will be discussed as well as where ongoing work is needed to further close this gap in dental education.

\section{OD03}

\section{Margin integrity and mechanical properties of selectively excavated and differently restored teeth in vitro}

Falk Schwendicke $^{1}$, Matthias Kern ${ }^{2}$, Christof Dörfer ${ }^{3}$, Julia KleemannLübkes $^{3}$, Uwe Blunck ${ }^{1}$, Sebastian Paris ${ }^{1}$

${ }^{1}$ Department of Operative and Preventive Dentistry, Charité Universitätsmedizin Berlin, Berlin, Germany, ${ }^{2}$ Department of Prosthodontics, Propaedeutics and Dental Materials, Christian-Albrechts Universität, Kiel, Germany, ${ }^{3}$ Department for Conservative Dentistry and Periodontology, Christian-Albrechts Universität, Kiel, Germany

Objectives: Selective (incomplete) caries excavation reduces the risk of pulpal complications, but might compromise the mechanical properties or marginal integrity of teeth or restorations. Different restorative approaches might be required after selective instead of complete excavation. We compared margin integrity, micro-leakage and fracture resistance of selectively excavated and differently restored premolars in vitro.

Methods: In 72 extracted human premolars, artificial residual lesions were induced on pulpo-axial walls of standardized cavities. Teeth were restored using one of three bonding systems (OptiBond FL, Clearfil SE Bond, G-aenial Bond) and a fiberenforced (EverX) or standard hybrid composite (Filtek Z250). After thermo-mechanical cycling, groups $(n=12)$ were compared regarding their gingivo-cervical margin integrity (proportion of acceptable margins), microleakage depth, and fracture resistance, with statistical evaluation using generalized linear modelling.

Results: Margin integrity was significantly influenced by the bonding system $(p<0.001)$, not the composite $(p=0.105)$. Proportions of acceptable margins were increased in teeth restored with OptiBond (median [25/ 75th percentiles]:93[78/100\%]) or Clearfil (82[60/94]\%) instead of Gaenial Bond (43[15/74\%]; $\mathrm{p}<0.05)$. Neither bonding systems nor composites significantly influenced leakage depth in enamel $(p=0.749 / 0.569)$ or dentin $(\mathrm{p}=0.112 / 0.909)$. Fracture resistance was significantly influenced by bonding system $(p=0.008)$ and composite $(p=0.001)$, and was higher in teeth restored using OptiBond (mean [SD]:1210[336]N) instead of Clearfil $(1007[208] \mathrm{N})$ or G-aenial Bond $(1023[281] \mathrm{N}, \mathrm{p}<0.05)$, and using EverX (1182[314]N) instead of Z250 (979[228]N; p < 0.01).

Conclusions: Bonding systems which perform well in completely excavated teeth also yielded good results for selectively excavated teeth in vitro. Using fiber-enforced composites to restore selectively teeth might increase fracture resistance.

\section{OD04}

Effect of Combining Radiographs and DIAGNOdent with Visual Examination on Detection of Occlusal Caries

Qasem Alomari, Muawia Qudeimat, Mai Khalaf, Yacoub Al-Tarakemah $\overline{\text { Kuwait University, Kuwait, Kuwait }}$

The aim of this laboratory study was to evaluate the effectiveness of incorporating radiographs and DIAGNOdent to visual examination on the detection and treatment of occlusal caries. Four examiners examined the occlusal surfaces of 160 extracted posterior teeth. Teeth were examined three times with a one month interval in between. The first examination was visual (V). The second examination was visual with radiograph (VR) and the third examination was visual with radiograph and DIAGNOdent (VRD). Examiners were asked to detect the presence and extent of caries. The examiners were also asked to choose a treatment for each tooth. The examined teeth were later sectioned and the presence of caries was charted. Sensitivity, specificity, area under the curve (Az values), rank correlation coefficient, and McNemar $\chi^{2}$ tests were calculated. VR had statistically higher specificity and lower sensitivity than $\mathrm{V}$ and VRD. The means of Az values ranged from 0.58 to 0.63 with no statistical difference between the three examinations $(\mathrm{p}>0.05)$. The means of the rank correlation coefficients with histology for detection of dentinal caries were: 0.48 , 0.23 and 0.44 using V, VR and VRD respectively. The percentages of teeth correctly treated according to histology were 69.4, 70.0 and 67.5 using V, VR, VRD respectively. There was no difference in the accuracy of treatment decisions between the 
three examination points $(\mathrm{P}>0.05)$. The addition of radiographs and DIAGNOdent to visual examination did not have a significant effect on the improvement of the detection and treatment decisions of non-cavitated occluso-dentinal carious lesions.

\section{OD05}

Airborne-particle Abrasion Is a Minimally Invasive Method to PreTreat Sclerotic, Non-Carious Cervical Dentin

Simon Flury, Anne Peutzfeldt, Adrian Lussi

Department of Preventive, Restorative and Pediatric Dentistry, School of Dental Medicine, University of Bern, Switzerland

\begin{abstract}
Aims: To investigate substance loss and bond strength capacity of sclerotic, non-carious cervical dentin after airborne-particle abrasion or diamond bur preparation.
\end{abstract}

Methods: Fifteen non-sclerotic dentin specimens were made from crowns of extracted human incisors of which the labial surfaces had been ground with silicon carbide papers (non-sclerotic control; Group 1). Forty-five sclerotic dentin specimens $(n=15 /$ group) were made from the labial, non-carious cervical root part of extracted human incisors and underwent either no pre-treatment (sclerotic control; Group 2), pretreatment with airborne-particle abrasion (CoJet System (3M ESPE) and $50 \mu \mathrm{m}$ aluminium oxide; Group 3), or with diamond bur preparation (40 $\mu \mathrm{m}$ grit size; Group 4). Substance loss after pre-treatment was measured in Groups 3 and 4. Subsequently, Scotchbond Universal (3M ESPE) and resin composite (CeramX (DENTSPLY DeTrey)) were applied on the treated dentin surfaces. The specimens were stored at $37^{\circ} \mathrm{C}$ and $100 \%$ humidity for $24 \mathrm{~h}$. After storage, shear bond strength (SBS) was measured and data analyzed with nonparametric ANOVA followed by Wilcoxon rank sum tests.

Results: Substance loss (medians) was $19 \mu \mathrm{m}$ in Group 3 and $113 \mu \mathrm{m}$ in Group 4. SBS-values (MPa; medians) in Group 2 (9.24) were significantly lower than in Group $1(13.15 ; \mathrm{p}=0.0069)$, Group $3(13.05 ; \mathrm{p}=0.01)$, and Group $4(13.02 ; p=0.0142)$. There was no significant difference in SBS between Groups 1,3 , and 4 ( $\mathrm{p} \geq 0.8063$ ).

Conclusions: Airborne-particle abrasion and diamond bur preparation restored bond strength of Scotchbond Universal to sclerotic dentin to the level of non-sclerotic dentin, with airborne-particle abrasion being less invasive than diamond bur preparation.

\section{OD06}

Non-invasive proximal adhesive restoration (NIPAR) compared to infiltration technique for treating initial proximal carious lesions

Marwa Abdelaziz ${ }^{1}$, Adele Lodi Rizzini ${ }^{1}$, Tissiana Bortolotto ${ }^{1}$, Albert Feilzer $^{2}$, ivo Krejci ${ }^{1}$

${ }^{1}$ University of Geneva, Geneva, Switzerland, ${ }^{2}$ University of Amsterdam, Amsterdam, The Netherlands

Objective: To compare a new technique (NIPAR) to the infiltration technique (ICON).

Methods: 13 extracted human posterior teeth with non-cavitated proximal carious lesions were cut vertically to obtain two symmetrical lesions.
Gr.1: half of the paired lesions surfaces $($ No $=13)$ were etched with $15 \% \mathrm{HCl}$ gel (120s) (ICON, DMH, Hamburg, Germany). Gr. 2: the second half $(\mathrm{No}=13)$ were abraded with Al2O3-coated metallic strips and etched with 37\% H3PO4 (120s). All samples were then stained with rhodamine isothiocyanate (RITC). After ethanol drying and isolation of the cut surface, Gr. 1 samples were infiltrated with ICON infiltrant (DMG) following manufacturer's instructions. Gr. 2 samples were infiltrated with Scotchbond Universal (3MEspe,MN,USA) for 180 s and coated with a thin layer of Tetric flow (Vivadent, Schaan, FL). After light curing, unbound rhodamine was bleached by immersion in $30 \%$ hydrogen peroxide $(12 \mathrm{~h})$. Remaining lesion pores were stained with sodium fluoresceine solution. Samples were observed with confocal microscopy (CLSM, Leica SP5-2P) and the percentage of infiltration (area of resin infiltration/area of total demineralization x100) was calculated using ImageJ (NIH,USA).

Results: 11 samples out of 13 showed greater penetration into the lesion for Gr.2 compared to Gr. 1 .The mean value of the penetration for Gr.1 was $20.1 \%$, while for Gr.2 it was $40.4 \%$. Statistical analysis (SPSS vers. 21 , paired t-test) revealed a significant difference between the 2 groups $(\mathrm{p}<0.05)$.

Conclusions: Within its limitation this study shows that using NIPAR represents a valid option for the early treatment of proximal lesions when compared to ICON.

\section{OD07}

Reasons for placement and replacement of direct restorative materials in Turkey.

Hakan Çolak $^{1}$, Çoruh Türksel Dülgergil ${ }^{1}$, Ertugrul Ercan ${ }^{1}$, Abdülkadir Şengün

${ }^{1}$ Ishik University School of Dentistry, Erbil, Iraq, ${ }^{2}$ Kirikkale University School of Dentistry, Kirikkale, Turkey

Aim: This study investigated reasons for the provision of direct restorations in postgraduate clinic at the University of Kırıkkale.

Methods: This study evaluated 5000 restorative procedures carried out on 1403 (59\%female and \%41 male) patients. The information recorded reported the patient's age, gender, tooth number, the class of restorations, the restorative material used, and the reasons for placement and replacement of amalgam and tooth-colored restorations. Statistical analysis was carried out using Chi-square test $(\alpha=0.05)$.

Results: Data were obtained on a total of 5000 restorations from $3672(73,4 \%)$ being initial placements and $1328(26,6 \%)$ replacements. The reasons for placement were principally primary caries $(88,1 \%)$, non-carious defects $(9,3 \%)$ and other reasons $(2,6 \%)$. Secondary caries was the most common reason for replacement ( $72 \%$ and $38,6 \%$ for composite and amalgam, respectively). The second major reason for the replacement restorations was poor anatomic form $(21.7 \%)$ for amalgam restoration and marginal discoloration for composite restoration. The resin composite was statistically $(\mathrm{p}=0.000)$ the most indicated material $(86.7 \%)$ for the first time placements.

Conclusion: Replacements have been the main reason for performing direct restorations, and the chosen restoration material was resin composite. 


\section{OD08}

\section{Analysis of cyclic thermal stress on restored tooth using finite element analysis}

Yoon Lee ${ }^{1}$, WooRham $\mathrm{Han}^{2}$, Ho-Bum Kwon ${ }^{2}$, Deog-Gyu Seo ${ }^{2}$

${ }^{T}$ Yonsei University Wonju Severance Christian Hospital, Wonju, Republic of Korea, ${ }^{2}$ Seoul National University School of Dentistry, Seoul, Republic of Korea

Introduction: The intake of hot and cold substances in daily life generates cyclic thermal stress to tooth, and this repeated stress may result in fatigue failure. The purpose of this study was to analyze the relationship between cyclic thermal stress and tooth crack formation using finite element analysis.

Methods: A maxillary first molar with class I or class II restoration was modelled via CAD-FEM procedure. Thermal load based on in vivo experimental data was applied to class I/II prepared natural tooth without filling material (control) and class I/II gold restored tooth. Stress generated in tooth structure was examined. Predicted fatigue life based on stress analysis was calculated using Basquin's equation.

Results: Calculated fatigue life was shorter in gold restored group than in controlled group and class II restored group showed shorter fatigue life than class I restored group. The shortest fatigue life was shown in class II gold restored tooth ( $3 \times 10^{7}$ years). For all cases, maximum stress was held in cold stimulation.

Conclusions: The cyclic thermal stress is a contributing factor of tooth crack formation and the presence of restoration facilitates the fatigue failure caused by cyclic thermal stress.

\section{OD09}

One year clinical performance of root surface restorations in older adults

Martina Hayes, Cristiane da Mata, Francis Burke, Finbarr Allen University College Cork, Cork, Ireland

Objectives: To compare the clinical performance of three restorative materials in root caries lesions.

Methods: A randomized clinical trial was conducted on independentlyliving older adults ( $>65$ years) in Cork, Ireland. Active, cavitated lesions on root surfaces were identified. Following caries removal they were restored randomly with one of three materials: (1) a packable glass ionomer (Fuji IX GP Extra, GC Corporation), (2) a resin modified glass ionomer (Fuji II LA, GC Corporation) and; (3) a tricalcium silicate based material (Biodentine, Septodont). Six and twelve months after placement the restorations were assessed by a blinded independent examiner according to USPHS criteria.

Results: 304 (86 GIC, 83 RMGIC, and 135 Biodentine) restorations were placed in 86 participants. At the time of this interim analysis, 254 restorations in 71 patients have been reviewed at 6 months, and 146 restorations in 41 patients have been assessed at 12 months. The 6 month survival rates were $54.3 \%$ for Biodentine, $82.6 \%$ for GIC and $91.3 \%$ for RMGIC. The 12 month survival rates were $33.3 \%$ for Biodentine, $80 \%$ for GIC and $85 \%$ for RMGIC.

There were statistically significant differences in restoration failure rates between the three groups at both 6 and 12 months (Chi-square test, $\mathrm{p}<$ 0.05 ). The success rates of GIC and RMGIC were similar but the success rate of Biodentine restorations was significantly lower than the other two materials $(\mathrm{p}<0.05)$.

Conclusion: The 6 and 12 month success rates of GIC and RMGIC were similar; however the success rate of Biodentine was significantly lower.

\section{OD10}

Can reduced-step polishers be as effective as multi-steps on enhancing surface smoothness?

Hande Kemaloglu, Gamze Yildirim, L. Sebnem Turkun

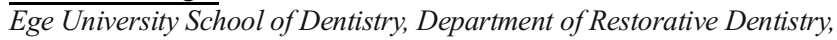
Izmir, Turkey

The aim of this study was to evaluate the effect of various reduced-step polishers versus multi-steps on the surface roughness of a resin composite. Ninety discs $(10$ x $2 \mathrm{~mm})$ of a nano-hybrid resin composite Tetric N-Ceram (Ivoclar/Vivadent, Liechtenstein) were fabricated in a metal mould covered with Mylar strip and randomly divided into 9 groups $(n=10)$. Except for the control group (Group 1), all the specimens were first finished with coarse and medium Super-snap discs (Shofu, Japan) and polished with the following systems as recommended by manufacturers: Shiny (Micerium, Italy) (Group 2), Venus Supra (Heraeus Kulzer, Germany) (Group 3), One-gloss (Shofu, Japan) (Group 3), Sof-Lex Spiral Wheels (3M ESPE, USA) (Group 4), Super-snap (fine, superfine) (Shofu, Japan) (Group 5), Pogo (Dentsply, USA) (Group 6), Clearfil Twist Dia (Kuraray, Japan) (Group 7), Rubber cups (Kenda, Liechtenstein) (Group 8). The discs were kept in water for one week before testing. The surface roughness (Ra, $\mu \mathrm{m}$ ) of all groups were measured with Mitutoyu SJ-301 (Mitutoyu, Japan) surface roughness tester. Results were statistically analyzed using One-way Anova and Dunnett T3 test at a $\mathrm{p}<0.05$ significance level.

Significant difference was found among the surface roughness of all groups $(\mathrm{p}<0.05)$. Super-snap, Pogo and Spiral-wheel groups presented similar results to the control group of Mylar strip with smoothest surfaces. These results were significantly different than Shiny, Venus, Clearfil Twist Dia and One-gloss groups $(p<0.05)$. The group that showed the roughest surface among all was the Rubber cup group ( $\mathrm{p}<0.05)$.

All the polishers were donated by their representative manufacturers.

\section{OD11}

Real bulk restorative technique with a dual cured bulk fill composite and one component adhesive

Tissiana Bortolotto ${ }^{1}$, Marwa Abdelaziz ${ }^{1}$, Ivo Krejci ${ }^{1}$

${ }^{T}$ Faculty of Medicine, University Clinic of Dental Medicine, Geneva, Switzerland, ${ }^{2}$ Faculty of Medicine, University Clinic of Dental Medicine, Geneva, Switzerland, ${ }^{3}$ Faculty of Medicine, University Clinic of Dental Medicine, Geneva, Switzerland

Objectives: Seeking to find a rapid restorative technique for class II cavities, this study aimed to: 
1. Evaluate marginal adaptation of a new dual cured bulk fill composite (Fill-Up!, ColtèneWhaledent, Altstätten, Switzerland) in combination with a one component SE adhesive (One Coat Universal, ColtèneWhaledent) in comparison to the original chemically cured adhesive system (Parabond, ColtèneWhaledent)

2. Determine if the use of a chemical activator is necessary for activation of One Coat Universal in combination with FillUp!

\section{Materials and Methods:}

Groups $(\mathrm{n}=8)$ :

1. OneCoat Universal with chemical activator

2. OneCoat Universal

3. Parabond

In box shaped bevelled MO cavities, adhesives were applied following manufacturer's instructions. Fill-Up! was injected in one layer, left undisturbed for $30 \mathrm{~s}$, and light cured from the occlusal for 20s. Percentages of continuous margins (Mean $\% \mathrm{CM} \pm \mathrm{SD}$ ) were quantified before / after thermo-mechanical loading (SEM)

Results: No significant difference between groups was detected for total marginal length after loading, \%CM ranging from $48.2 \pm$ 13.7 to $68.6 \pm 8.5$ (ANOVA; $p>0.05$ ). On cervical margins (critical area due to maximum distance from curing light), Gr. 1 attained significantly highest \%CM $(85.0 \pm 10.1)$, followed by Gr. $3(44.1 \pm 27.2)$. Gr. 2 resulted in poor marginal adaptation $(\% \mathrm{CM}: 10.0 \pm 8.8)$.

Conclusions: High \%CM were attained in class II restorations filled with a bulk fill dual cured composite and a one component SE adhesive. However, the use of an activator was mandatory to compensate for lack of light in the depth of the cavity.

\section{OD12}

comparing self-etch adhesives for the infiltration of non-cavitated carious lesions

Marwa Abdelaziz ${ }^{1}$, Adele Lodi Rizzini ${ }^{1}$, Albert Feilzer ${ }^{2}$, Ivo Krejci ${ }^{1}$

${ }^{1}$ University of Geneva, Geneva, Switzerland, ${ }^{2}$ ACTA, Amsterdam, The Netherlands

Objective: to compare the efficiency of infiltration of three self-etch adhesives (one or two-component) into natural non-cavitated carious lesions.

Methods: 21 extracted human molars and premolars with non-cavitated interproximal lesions were selected. ICDAS code 1-2 was assessed by visual and microscope analysis. Samples were cut vertically across the demineralization ( $\mathrm{n}=24$ lesions). After isolating the cut surface, lesion surfaces were abraded with fine A12O3-coated metallic strips and etched (H3PO4 37\% 120s). Teeth were stained with rhodamine-isothiocyanate (RITC) solution (12h) and dried (ethanol 96\% 30s, air-dry 10s).Lesions were randomly divided into three groups and infiltrated with self-etch adhesive systems. Gr.1: one-component One-Coat7.0 (Coltene/ Whaledent AG, Switzerland); Gr.2: one-component Scotchbond Universal (3MEspe,MN, USA); Gr.3: two-component Clearfil Protect Bond
(Kuraray Noritake Dental Inc., Okayama, Japan).In all groups the adhesive was applied twice (180s, light-cured, 60s) and coated with a thin layer of (Tetric flow,Vivadent, FL). After light curing, unbound dye was bleached by immersion in hydrogen peroxide $\left(30 \% 12 \mathrm{~h}, 37^{\circ} \mathrm{C}\right)$. Remaining lesion pores were stained with sodium fluorescein solution. Thin cuts of the teeth were observed with confocal microscopy (LSM510 Meta, Zeiss, Germany) and computer image analysis was performed (ImageJ, $\mathrm{NIH}$, USA) to calculate the percentage of infiltration (resin infiltration area/total demineralization area $\mathrm{x} 100$ ).

Results: Infiltration with the three different self-etch adhesives showed similar results. The mean percentage of infiltration was (Gr.1) $27.7 \%$, (Gr.2) 22.7\%, (Gr.3) 24.6\%.

Conclusions: Within the limitation of this in vitro study, one-component and two-component self-etch adhesives are equally suitable for infiltration of natural-caries lesions.

\section{OD13}

Comparison of conventional vs spectrophotometric methods of shade selection regarding experience

Duygu Recen, Banu Onal, L. Sebnem Turkun, Hazal Cankurt Ege University School of Dentistry, Izmir, Bornova, Turkey

Purpose: The objective was to compare the shade selection ability among dentists with different levels of experience in esthetic dentistry in comparison to a spectrophotometer.

Methods: A clinical undergraduate student, a research assistant with 2 years of experience and two restorative dentistry specialists with more than 10 years of experience were included in the study as examiners. The participants were selected from volunteer students of Ege University School of Dentistry $(n=100)$. All the subjects were healthy with no bleaching history and were caries/restoration free on their anterior teeth. The shade of tooth $\# 11$ of all students was taken by the examiners one by one-using Vita Classical (Vita Zahnfabrik, Bad Sackingen, Germany) shade tab. At the end, the research assistant took the shades again with SpectroShade Micro (MHT Optic Research, Niederhasli, Switzerland) device. The color codes obtained were statistically evaluated compared to the device and among them with Wilcoxon Signed Ranks Test with a significance level of .005.

Results: The difference in scores among the experienced examiners was not statistically significant. However, the research assistant could guess the shade of the teeth better than the dentistry student $(\mathrm{p}<0.05)$ and as she was familiar with the device, was the only one who could guess the shades similarly to the Spectrophotometer.

Conclusion: According to the results of this study, it can be said that conventional shade matching ability is increasing with experience. However for inexperienced dentists, Sepctrophotometer devices may be helpful to confirm the shade selected with classical shade guides.

\section{OD14}

Microleakage Evaluation of Direct Composite Inlay, Indirect Porcelain Inlay and Teeth Restored with Cad/Cam Systems

Damla Ibrahimov, Ertugrul Ercan

Kirikkale University, Faculty of Dentistry, Kirikkale, Turkey 
Aim: The aim of this study was to evaluate in vitro edge leakage of inlay applications that were restored with using different methods and materials.

Materials and Methods: In this study, class II mesio-occlusal cavities were prepared to 80 caries and restoration free human third molar teeth. The teeth were divided into four groups and 20 teeth in each group. 1.Group: Filtek Ultimate direct composite resin inlays, 2.Group: Lava Ultimate inlays, 3.Group: IPS e.max Press ceramic inlays and 4.Group: IPS e.max $C A D$ inlays were prepared. After finishing and polishing procedures 10.000 thermal cycle between $5 \pm 1{ }^{\circ} \mathrm{C}$ and $55 \pm 1{ }^{\circ} \mathrm{C}$ was applied to all specimens. All samples were suspended in a $0.5 \%$ basic fuchsin solution for 24 hours. After, the teeth were cut longitudinally. Photos of dye penetration scores were recorded at $\mathrm{x} 30$ magnification on the computer.

Results: According to the results, in occlusal region; lowest microleakage values were identified in e.max CAD and Lava groups, than Filtek and e.max Press groups, respectively. Groups prepared in occlusal regions by using $\mathrm{Cad} / \mathrm{Cam}$ methods showed lower microleakage values than the other groups and the difference was statistically significant $(p<0.05)$. In gingival region compared between the groups, there was no significant difference between materials $(\mathrm{p}>0.05)$.

Conclusion: When microleakage values compared, CAD / CAM generated inlay groups gave better results; but in cervical regions, no significant difference was determined between different groups. However, further clinical studies are needed.

\section{OD15}

Evaluation of dentinal tubule occlusion by Nd:YAG and Er:YAG lasers: Real-time measurement of dentinal fluid flow

Inbog Lee, Minho Kim, Ryan Kim

School of Dentistry, Seoul National University, Seoul, Republic of Korea

Objectives: The objective of this study was to evaluate the effect of $\mathrm{Nd}$ :YAG, Er:YAG lasers and a topical desensitizing agent on dentinal tubule occlusion by measuring real-time dentinal fluid flow and observing the tubule surfaces.

Methods: Twenty-four molars were prepared with V-shape cavity at the cervical area, acid etched, water rinsed, blot dried, and treated with 1) Nd:YAG laser; 2) Er:YAG laser; and 3) SuperSeal, a desensitizing agent $(n=8)$. A real-time fluid flow measuring device (nano-Flow) was used to measure the dentinal fluid flow throughout the procedures. The fluid flow rates before and after the lasers or desensitizing agent application were compared. The surface topography of dentinal tubules after each desensitizing method application was examined using SEM.

Results: The dentinal fluid flow varied among the groups. The fluid flow rate was significantly reduced after the lasers or desensitizing agent application $(p$ $<0.05)$. The reduction of fluid flow rate in SuperSeal group $(34.8 \%)$ was significantly higher than in Nd:YAG (24.1\%) and Er:YAG (20.6\%) groups ( $p$ $<0.05)$. There was no statistical difference between two laser groups $(p>$ $0.05)$. In SEM images, the narrowed dentinal tubules by the melted dentin in the laser groups and by the oxalate crystals in SuperSeal group were observed.

Significance: The dentinal fluid flow rate was significantly reduced after the lasers or desensitizing agent application. SuperSeal group exhibited better reduction in dentinal fluid flow rate than did other two laser groups.

\section{OD16}

22-Months Survival of Deep Dentin Caries Lesions: A Retrospective Clinical Study

Sukran Bolay, Fatma Dilsad Oz, Ceren Ozge Bicer, Elif Ozturk Hacettepe University, Ankara, Turkey

Objectives: The aim of this retrospective study was to evaluate the longterm clinical survival of stepwise versus complete excavation in deep dentin caries lesions.

Methods: One hundred-three patients (35-male,68-female) with at least one permanent tooth with clinically diagnosed deep dentin caries were included. Inclusion criterias were teeth with deep caries lesions having a radiolucency halfway or more into dentine and pulp vitality but absence of spontaneous pain, positive percussion test, and periapical alterations. The subjects received either stepwise (SW) or complete excavation (CE). The radiological and clinical exams were performed after a mean observation time of 22 months. Success was defined as pulp sensitivity to vitality test and absence of periapical lesions as well as a clinical symptom. Data were statistically analyzed using Kaplan-Meier and Log-Rank (Mantel-Cox) tests (SPSS20.0, $\alpha=0.05$ ).

Results: Of the 108 treatments performed, 84 were SW and 24 were CE. Fifty-eight restorations were amalgam, 41 were composite and 9 were resin modified glass-ionomer cement. Mean observation period was 22 months. Survival rates were 91 and $95 \%$ for SW and CE respectively after 12 months $(p=0.356)$. For the SW group, survival rate was $88 \%$ at 22 months whereas this rate decreased to $70 \%$ at 52 months. At 39 months, survival rate decreased to $88 \%$ in CE group. Nine of the failed restorations were glass-ionomer. There was a significant difference between survival rates of glass-ionomer and other 2 restoration groups $(p=0.043)$.

Conclusions: Pulp vitality was not adversely effected by either stepwise or complete excavation after a mean 22 months observation.

\section{OD17}

Influence of different shade of luting cements on feldspatic and lithium-disilicate veneers of varying thicknesses

Michele Ribero, Allegra Comba, Damiano Pasqualini, Mario Alovisi, Elio Berutti, Nicola Scotti

university of turin, turin, Italy

Objectives: The aim of this in vitro study was to assess the influence of different luting cement shade on color predictability of feldspatic and lithium-disilicate ceramics. The null hypothesis tested is that the luting cement do not influence the final shade of ceramic, independently from the thickness and the ceramic employed.

Methods: 120 composite (Adonis,Sweden and Martina) discs, shade 4 , of $2 \mathrm{~mm}$ thickness were prepared and used as background to simulate natural tooth. 120 ceramic discs (A2-HT) were prepared and divided into 2 groups $(n=60)$ according to the material used: A)lithium-disilicate B)feldspatic ceramic. Samples of each group were than divided into three subgroups $(n=20)$ according to the ceramic thickness: $0.6 \mathrm{~mm} ; 1.0 \mathrm{~mm} ; 1.5 \mathrm{~mm}$. Each sample was than applied on the composite background using luting material with different shade: a) no cement; b) Yellow (NX3, Kerr); c)Clear (NX3); d)Milky Bright (Choice2,Bisco); e)A2 (Choice2). The resulting sample shade was assessed using a 
spectrophotometer (Spectroshade,MHT) on white and black background. Analysis of variance $(\mathrm{p}<0.05)$ was performed to evaluate the effect of different luting material shade(1), ceramic type(2) ceramic thickness(3) and their interaction on final shade.

Results: ANOVA showed that, only with samples analyzed on white background, ceramic thickness influence the final sample shade. Luting material A2 and Milky Bright color seems to statistically influence ceramic shade only when its thickness is $0.6 \mathrm{~mm}$. No differences were found between the 2 different ceramics tested.

Conclusions: The null hypothesis is rejected since ceramic thickness with some opaque color of cement influences the final shade of translucent ceramics.

\section{OD18}

A novel protocol for predictable placement of prefabricated veneers.

Dimitrios Spagopoulos, Efstratios Papazoglou

Department of Operative Dentistry, Dental school, University of Athens,, Athens, Greece

Even though the prefabricated veneers promise good esthetics due to their prefabricated surface, they fail to present a predictable clinical outcome. The manufacturing companies of prefabricated veneers propose a single appointment procedure without the ability to realize a mock up, showing the patient the desired final result.

A novel 2-appointment protocol with a laboratory step was designed in order to be able to achieve: (a) a mockup, (b) a tooth reduction guide and, (c) a controlled veneer placement.

During the first appointment, photographic documentation and initial impressions are taken to evaluate the case and create a personalized treatment plan. The size of the prefabricated veneers is selected by applying the Sizing Guide on a stone model. Depending on the clinical situation, a combination of sizes can be used. The selected prefabricated veneers are then adapted to the stone model by minimally preparing the gypsum teeth. After finishing the preparation of the teeth, silicon reduction keys are created for transferring these preparations intraorally. This is a clinical sensitive and time consuming technique, however, it preserves sound tooth structure by reducing only the necessary tooth volume. Consequently, the prefabricated veneers are stabilized on the prepared stone model with wax and silicone transfer keys are fabricated. Finally, the shade for the luting composite resin is selected.

During the second appointment, the mock up is realized first. After patient approval of the designed treatment plan, the rest of the procedure is totally predictable using the aforementioned silicon keys for tooth reduction and veneer placement.

\section{OD19}

Importance of conditioner on marginal sealing of cavity fillings using Sonicfill ${ }^{\mathrm{TM}}$

Sebastiana Arroyo Bote, Javier Martinez Martinez Osorio Faculty of Dentistry Barcelona University, Barcelona, Spain
Introduction: The development of composites with low polymerization shrinkage and increased depth of cure could help foster the quality of marginal sealing. Nevertheless, the lower effectiveness of self-etch adhesives on enamel could compromise the resistance of the sealing.

Objective: The aim of the study was to compare the marginal leakage in class I cavities sealed with SonicFill ${ }^{\mathrm{TM}}$ and OptiBond XTR with and without conditioner.

Material and Methods: We performed class I cavity in 20 extracted human molars free of caries, divided into two groups A and B:

A Filling with OptiBond XTR and SonicFill ${ }^{\mathrm{TM}}$

B Engraved with $37 \%$ acid for 15 ' enamel + filling with OptiBond XTR and SonicFill ${ }^{\mathrm{TM}}$

After sealing of the root and storage in artificial saliva, the samples were immersed in methylene blue for 24 hours. After that, the samples were subsequently washed. Finally, a longitudinal cut was performed dividing the sample into two halves that were observed with optical microscope.

Results: In order to analyze the results, we defined the following filtration values

0 No filtration

1 Filtration in enamel

2 Filtration in the dentin side wall

3 Filtration in the cavity floor

Statistical analysis shows a significant difference between the two groups, performing better group B.

Discussion-Conclusion: Composites with low polymerization shrinkage present a lower stress to polymerization, but it is important to use adhesive with sufficient adhesion to ensure the resistance of the sealing. Results demonstrate that previous engraving of enamel significantly improves marginal sealing in cavities filling with SonicFill ${ }^{\mathrm{TM}}$ and OptiBond XTR.

\section{OD20}

Evaluation of self-adhering materials as desensitizing agents in xerostomic patients with head and neck cancer.

Roberto Pinna, Giovanni Sotgiu, Simone Dore, Egle Milia University of Sassari, Sassari, Sardinia, Italy

Aim: To evaluate the desensitizing capacity of four different materials to treat dentine hypersensitivity in xerostomic patients due to radiotherapy for head and neck cancer.

Methods: A total of 68 patients underwent to radiotherapy were selected for the study. The study was conducted as a split-mouth randomized clinical trial, comparing: Vertise Flow ${ }^{\mathrm{TM}}$, Universal Dentine Sealant, Clearfil Protect Bond and Flor-Opal ${ }^{\circledR}$ Varnish. Basal and stimulated salivary flow was recorded for each patient according to European classification criteria (1993). The pain experience was generated by a cold stimulus directly to sensitive tooth surface and assessed using the Visual Analogue Scale (VAS). The response was recorded before the application 
of the materials (PRE-1), immediately after (POST-1), at 1 week (POST2), 4 weeks (POST-3) and 12 week controls (POST-4).

Results: The number of patients meeting the inclusion criteria was 24 . The mean basal salivary flow rate was $0.24 \mathrm{ml} / \mathrm{min}$ (minimum $0.06-$ maximum 0.42 ), while the stimulated rate was $0.54 \mathrm{ml} / \mathrm{min}$ (minimum 0.29 - maximum 0.86). All the materials tested produced a significant reduction in the dentine sensibility at POST-1 and POST-2. However, at POST-4, showed a dramatically decrease of performance and reduction of the VAS scores have returned close in a similar manner to those measured at the PRE-1.

Conclusion: As a result of this investigation, in xerostomic condition patients all the materials tested produced a reduction of dentine hypersensitivity. However, after 12-week controls, there was no significant difference in the decrease of the VAS irrespective of the desensitizing agent employed.

\section{OD21}

Does the Silane in Multi-mode Universal Adhesive function to Lithium Disilicate?

Hyunyoung Lee, Byeong-Hoon Cho, Ho-Hyun Son

Department of Conservative Dentistry, School of Dentistry and Dental Research Institute, Seoul National University, Seoul, Republic of Korea

Purpose Multi-mode universal adhesives (MUA) containing many ingredients were introduced in dentistry. We studied the influence of silane in MUA on the bonding of resin cement to lithium disilicate through microshear bond strength ( $\mu \mathrm{SBS})$.

Materials and methods Twenty $12 \times 14 \times 5 \mathrm{~mm}$ E. $\max \mathrm{CAD} / \mathrm{CAM}$ specimens for each group were fabricated and sintered. Each specimen was embedded into acrylic resin and polished to 500 grit. The surfaces of specimens were treated as follows. Group A: adhesive not-containing silane (ANS); Group B: saline (S) and ANS; Group C: 4\% hydrofluoric acid (HF), S and ANS; Group D: adhesive containing silane (ACS); Group E: 4\% HF and ACS. Dual-cure resin cement (NX3; Kerr) was applied and prefabricated composite resin cylinder (diameter $0.8 \mathrm{~mm}$; Filtek Z250, 3M EPSE) was placed on the surface. The cement was light-cured for 40 seconds. All samples were stored in distilled water at $37^{\circ} \mathrm{C}$ for 24 hours, and were submitted to shear testing.

Result Bond strengths were significantly different among each groups ( $\mathrm{p}$ $<0.05$ ) except for Group A and D. The mean $\mu$ SBS (MPa) were; A: 1.18 \pm 1.02 ; B: $8.85 \pm 2.67$; $: 26.90 \pm 6.92$; D: $1.32 \pm 0.60$; E: $21.36 \pm 5.24$. The $\mu$ SBS of Group E was significantly lower than that of Group C. Fracture modes were analyzed: predominantly adhesive in non HF treated groups and cohesive in HF treated groups.

Conclusion The silane in MUA seems not to influence on the bonding to lithium disilicate. The surface treatment using HF, silane and adhesive (ANS) is recommended in bonding of resin cement to lithium disilicate.

\section{OD22}

Tooth-composite interaction of self-adhering flowable composites

Christina Brückner, Hartmut Schneider, Rainer Haak

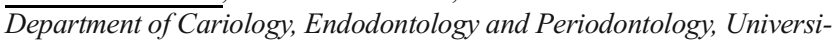
ty of Leipzig, Leipzig, Germany
Purpose: Evaluation of microleakage and tooth-composite interaction of self-adhering flowable composites.

Materials and Methods: Standardized class V restorations were prepared (3 mm incisal-apical, $4 \mathrm{~mm}$ mesio-distal and $1.5 \mathrm{~mm}$ in depth) at 32 human molars. Per group 8 teeth were restored with Vertise Flow (VF), Fusio Liquid Dentin (FLD), an experimental flowable (EF) and the restoration system Adper Prompt-L-Pop/Filtek Supreme XT Flowable (PLP, control). The teeth were prepared according to laboratory standard, stored in distilled water $\left(24 \mathrm{~h}, 37^{\circ} \mathrm{C}\right)$ and microleakage (ML, percentage of interface length at enamel/dentin) and tooth-composite interaction were determined by light and electron microscope. Data were analyzed using Mann-Whitney U test ( $\alpha=0.0042$; Bonferroni adjustment, trend: $\alpha<\mathrm{p}<0.05$ ).

Results: a) Microleakage formation: At enamel, less ML occurred with VF $(10.3 \%)$ and EF (9.0 \%) compared to FLD (52.9 \%; p = 0.009/p < $0.001)$ and PLP $(82.1 \%$; all $\mathrm{p}<0.001)$. At dentin, less penetration was found with VF (17.6\%) and FLD (4.4\%) compared to EF $(75.2 \%$; $\mathrm{p}=$ $0.01 / \mathrm{p}<0.001)$ and PLP $(62.3 \% ; \mathrm{p}=0.002 / \mathrm{p}<0.001)$.

b) Tooth-composite interaction: Adhesive tags at enamel were verifiable constantly with EF and VF, whereas these occurred irregularly with FLD and PLP. At dentin, tags were detectable with all systems.

Conclusion: The current study showed an inverse relation between microleakage formation (sealing) and microretentive anchoring of the restoration to enamel. Based on this, the control system PLP seems to be inferior to the self-adhering flowables.

\section{OD23}

Composite onlays constructed with semidirect technique in mounted silicone casts

Nektarios-Nikolaos Vasilopoulos, Dimitra Kafetzi, Michalis Diomataris, Sofia Diamantopoulou, Efstratios Papazoglou

School Of Dentistry, National and Kapodistrian University of Athens, Athens, Greece

Patients' demand for aesthetic metal-free restorations in the posterior region is continuously increasing. The evolution in adhesive dentistry has broadened the indication for resin composite materials. The primary disadvantage of composite resin, is polymerization shrinkage in the direct technique. To overcome this important disadvantage two techniques have been developed, the indirect and the semidirect technique. The indirect technique requires two appointments and a laboratory involvement. In contrast, in the semidirect technique the onlay can be prepared chairside in a single visit either conventionally or by the use of computer-aided design/computer-aided manufacturing systems (CAD/CAM).

A modified procedure for the conventional semidirect technique is described here. First the cavity is managed and then alginate impressions of the maxilla and mandible are taken. The impression with the prepared tooth is poured with special very rigid addition type silicon material (Mach-2, Parkell, USA) only in the prepared tooth area including two adjacent teeth. The rest of the impression, as well as the impression of the opposite arch are poured with quick setting mounting gypsum. Afterward, the casts are articulated on a special gypsum-free articulator (MagicArt-2, Alphadent Co, Seoul, Korea). The onlay is fabricated and post-cured using light and heat. The occlusion is first checked on the articulator and then the onlay is bonded into the patient's mouth, followed by the final check of the occlusion and polishing of the restoration. 
The use of this technique combines the advantages of the direct and the indirect approach with full control of form, shade and occlusion.

\section{OD24}

Marginal adaptation of class $\mathrm{V}$ cavities prepared with a Quantum Square Pulse Er:YAG laser

Izabella Nerushay, Anastasia Ryabova, Ivo Krejci

University of Geneva, Geneva, Switzerland

Objective: To evaluate marginal adaptation of mixed class V composite restorations in cavities prepared with the new highly efficient Quantum Square Pulse (QSP) mode Er:YAG laser (LightWalker, Fotona), compared to diamond bur preparation.

Methods: Best laser parameters with the noncontact handpiece were determined in a pretest by SEM micromorphological evaluation of enamel and dentin by varying pulse length, power and water spray settings. 21 extracted caries-free human third molars were subsequently randomly equally distributed into Gr. 1: Laser $300 \mathrm{~mJ} / 12 \mathrm{~Hz}$; Gr. 2: Laser $300 \mathrm{~mJ}$ followed by $120 \mathrm{~mJ} / 12 \mathrm{~Hz}$ and Gr. 3: Diamond bur. All cavities were restored with Clearfil SE Bond (Kuraray) and Clearfil AP-X (Kuraray) composite under dentinal fluid simulation. SEM marginal adaptation analysis at 200x magnification was performed on replicas before and after thermo-mechanical cyclic loading (TML).

Results: The mean \% of "continuous margin" before/after TML in enamel were Gr.1: 86.7/80.6; Gr.2: 95.7/88.6; Gr.3: 96.1/93.1 (ANOVA; $p$ > 0.05/>0.05) and in dentin Gr.1: 97.3/88.5; Gr.2: 96.7/93.4; Gr.3: 91.6/ 81.0 (ANOVA; $\mathrm{p}>0.05 />0.05$ )

Conclusions: No significant differences in \% "continuous margin" were detected for laser prepared cavities in comparison to bur prepared cavities, both in enamel and dentin, before and after loading. Among the two laser groups, there was a trend towards better marginal adaptation in enamel with $300 \mathrm{~mJ} / 120 \mathrm{~mJ}$ compared to $300 \mathrm{~mJ}$ only.

\section{OD25}

Influence of Carbodiimide Cross-Linking on bond strength stability of universal adhesive to radicular dentin

Guido Gregori

University of Turin, Dental School, Turin, Italy

Objective: The aim of this in vitro study was to evaluate the effect of a cross-linker agent (carbodiimide, EDC) used as additional primer for fiber posts cementation to radicular dentin. The null hypothesis tested was that EDC do not influence fiber post bond strength stability overtime when employed with a universal adhesive.

Methods: 24 extracted intact single-root teeth were selected, endodontically treated and a $10 \mathrm{~mm}$ post-space was then prepared. Samples were randomly divided into 2 groups $(\mathrm{n}=12)$ according to the adhesive protocol: 1)AllBond Universal (Bisco); 2) $0.3 \mathrm{M}$ EDC + AllBond Universal. The adhesive was applied with the etch-and-rinse protocol and EDC was used after phosphoric acid etching for $1 \mathrm{~min}$ and prior bonding application. Fiber posts were luted using a dual-cure resin cement (Duo-Link Universal, Bisco). Samples of each group where than divided into two subgroups ( $\mathrm{n}$ =6): a) immediate push-out test; 2) 12 months storage in artificial saliva and push-out. To perform push-out test, of the coronal and apical region, teeth were cut in $1 \mathrm{~mm}$-thick slices and pushed until failure with an Instron Machine. Results were statistically analyzed with ANOVA test.

Results: ANOVA showed that independently from aging and EDC pretreatment the coronal region showed statistically higher bond strength than the apical one $(p=0.0001)$. Aging, EDC pretreatment and the interactions between them did not improve bond strength.

Conclusions: The null hypothesis has to be rejected since EDC did not influence bond strength stability of universal adhesive employed for fiber post cementation, both before and after artificial aging.

\section{OD26}

Comparison of optical characteristics of resin composite by the different finishing and polishing system

A-Ra Jo, Sung-Eun Yang

Department of Conservative Dentistry, Seoul St. Mary's Dental Hospital, Catholic University of Korea, Seoul, Republic of Korea

Objective: Translucency and fluorescence are important factors of esthetic anterior restoration. In composite restoration, finishing and polishing system might have an influence on their optical characteristics. The objective of this study was to compare the translucency and fluorescence of nano-hybrid resin composites after various finishing and polishing systems

Materials \& Methods: 28 specimens $(15 \mathrm{~mm}$ diameter, $2 \mathrm{~mm}$ thickness) of nano-hybrid composite (Z350/A2, 3M ESPE) was sorted into 4 groups of various finishing and polishing $\operatorname{systems}(\mathrm{n}=7)$; Sof-Lex(SL, 3M ESPE), Enhance/PoGo(EP, Dentsply Caulk), Venus Supra(VS, Heraeus Kulzer), negative control Mylar strip(CL). Translucency Parameter(TP) and fluoresecence(FL) of specimens was measured using a reflection spectrophotometer(color-eye 7000A) with SCE/SCI geometry under the D65 illumination over white and black backgrounds. The values of TP, FL were measured after thermocycling again, for comparing optical characteristics of resin composite between different finishing and polishing systems.

Results: EP group showed the lowest TP value. There was no significant difference between group SL, VS and CL. The Value of TP showed no significant differences $(\mathrm{p}>0.05)$ in all groups between before and after thermocycling.

CL showed the highest FL before thermocycling. After thermocycling, fluorescence of test group significantly was increased. SL and VS group showed significantly higher FL value compared to EP and CL group.

Conclusion: SL and VS system exhibited similar translucency and fluorescence with human dentin. Within the limitation of this study, SL and VS system would be clinically acceptable with regard to optical characteristics. 


\section{OD27}

Prospective Clinical two year Split-mouth-study comparing Flowable Composites in Class-V-Restorations

$\underline{\text { Sabine May }}^{2,1}$, Karl-Anton Hiller ${ }^{1}$, Gottfried Schmalz ${ }^{1}$, Wolfgang Buchalla ${ }^{1}$, Marianne Federlin ${ }^{1}$

${ }^{1}$ University of Regensburg Dental School, Department of Conservative Dentistry and Periodontology, Regensburg, Germany, ${ }^{2}$ Private Praxis, Amberg, Germany

Objectives: To evaluate the clinical performance of two flowable composites for restoring Class-V non-carious-cervical-lesions (NCCLs), one with (ND: N'Durance Dimer Flow;Septodont), one without (FS: Filtek Supreme XTE Flow;3MEspe) phase separation and reduction in polymerization shrinkage stress. The Null-hypothesis was that both composites perform equally regarding clinical quality and survival.

Methods: In this controlled, prospective, clinical split-mouth study 50 patients (16male/34female) received one ND and one FS Class-V restoration of NCCLs each in premolar teeth, using Clearfil Protect Bond (Kuraray) as adhesive. The restorations were clinically rated by two examiners using FDI criteria at baseline, 12 and 24 months. Criteria evaluated were surface lustre, staining surface, staining margin, fracture material and retention, marginal adaptation, postoperative hypersensitivity, tooth vitality. Gingival status was evaluated. Non-parametric statistical analyses and chi-square tests were applied $(\alpha=0.05)$.

Results: One ND restoration failed between BL and 12 months. The remaining 49 patients with both restorations under risk participated in all recalls. Median patient age was $49(20-65)$ years, median PBI at BL was $16 \%$ (maximum 29\%). All 49 ND and FS restorations were rated clinically acceptable. In one patient, both restorations showed intense hypersensitivity at BL which decreased to normal sensitivity after 12 months. No significant differences for all criteria were recorded between ND and FS at each examination. For each material, no significant differences among BL and 12 and $\mathrm{BL}$ and $24 \mathrm{mo}$ were detected, except for loss of surface-lustre (BL to 12mo).

Conclusions: Within the limitations of the study, the Null-hypothesis (materials perform equally) could not be rejected.

\section{OD28}

3-Years clinical outcome of direct pulp capping performed with different resin-based materials

Marco Cuppari, Francesco Petrolo, Giulia Massano, Mario Alovisi, Damiano Pasqualini, Elio Berutti, NIcola Scotti

University of Turin, Dental School, Torino, Italy

Objectives: To evaluate the influence of three adhesive resin-based materials in the clinical outcome of direct pulp capping. The null hypothesis is that different resin-based materials do not influence the outcome of teeth with pulp exposion

Methods: Sixty patients with one asymptomatic primary carious lesion which showed pulp exposure during cavity cleaning were selected and included for this in-vivo study. Direct pulp capping was randomly performed with three different materials $(\mathrm{n}=20)$ : (G1)self-etch adhesive system (ProtectBond, Kuraray), (G2)glass-ionomer cement (Fuji IX, GC) and (G3)light-cured MTA-like material (TheraCal, Bisco), that were applied on exposed pulp according to the manufacturer's instructions. Direct restoration with a nanohybrid resin composite (Venus Diamond, Heraeus Kultzer) was then performed. Patients were recalled at one week, six months, 1, 2 and 3 years follow-up. All data collected were statistically analyzed with a $\chi^{2}$ test $(\mathrm{p}<0,01)$.

Results: After 3-years of clinical function, in Group 1 teeth were vital at $83,3 \%$, in Group 2 at $66,6 \%$ and in Group 3 at $93,3 \%\left(\chi^{2}=10,3\right.$ on vitality maintenance, greater than Group 1 and 2). Bleeding and preoperative symptoms were significantly associated to pulpal necrosis, while pulpal exposure was not significantly correlated to pulp capping outcome.

Conclusions: Based on the results null hypothesis is rejected, confirming a significant correlation between materials and pulpal vitality preservation. The use of TheraCal seems to significantly influence the clinical outcome of teeth with pulp exposure. Further follow-up controls and an increased number of patients are necessary to confirm the results of this in-vivo study.

\section{OD29}

Fracture resistance of endodontically treated teeth. An in vitro comparison between two post restoration techniques

Paula Gómez-Pérez, Vicente Faus-Matoses, Teresa Alegre-Domingo, Vicente J. Faus-Llácer

University of Valencia. Master in Restorative dentistry and Endodontics, Valencia, Valencia, Spain

Introduction: Endodontically treated teeth preserve a small amount of residual dentin, increasing the risk of fracture than vital teeth. The aim of this in-vitro study was to evaluate the fracture resistance of endodontically treated premolars restored with single or double-post restoration technique.

Material and methods: Seventy extracted mandibular premolars were selected. The specimens were decoronated at the cementoenamel junction and endodontically treated. The samples were divided in 2 groups $(n=35)$; one group restored with singlepost technique and the other with double-post technique. The fiber posts of Core\&Post System (Dentsply DeTrey, Konstanz, Germany) were cemented with composite cement CoreXflow (Dentsply DeTrey, Konstanz, Germany). All teeth were restored with composite restorations and submitted to the fracture resistance test in a universal testing machine until fracture. Data were statistically analyzed using Kolmogorov-Smirnov and Levene's tests and by independent $\mathrm{t}$-test at a significance level of 5\%.

Results: Double-post restoration technique filled more efficiently ovalshaped canals, reducing the amount of cement needed to fill the gaps between the post and dentin. Double-post technique induced a reduction of root fracture risk than single post-technique.

Conclusion: Within the limitations of this study, teeth with double-post restoration may show a reduction of failure loads than those with singlepost restorations. 


\section{OD30}

Fracture resistance of three restoration techniques: An in-vitro comparative study.

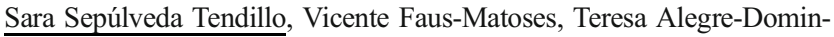
go, Vicente J. Faus-Llácer

Valencia University. Master in Restorative Dentistry and Endodontics, Valencia, Valencia, Spain

The aim of this in-vitro study was to compare the fracture resistance of endodontically treated premolars with fiber post and posterior reconstruction with core-build-up, bulkfill or incremental technique.

Ninety nine extracted free-decay mandibular premolars were decoronated above $2 \mathrm{~mm}$ to cementoenamel junction and endodontically treated. Canals were prepared with a rotatory crown-down technique and filled with thermoplastic technique. Post spaces were prepared leaving $5 \mathrm{~mm}$ of the endodontic filling to reach the apex of each canal. The specimens were divided into three groups $(\mathrm{n}=33)$ according to the cement used for post cementation. Group 1: Post cementation with Prime \& Bond NT+ Self cure Activator (Denstply, DeTrey, Konstanz, Germany), Calibra (Denstply, DeTrey, Konstanz, Germany) and incremental restoration with resin composite. Group 2: Post cementation with Prime \& Bond NT and Surefil $^{\circledR}{ }^{\circledR} \operatorname{SDR}^{\mathrm{TM}}$ (Dentsply, Maillefer, Ballaigues, Switzerland) and bulkfill restoration with Surefil ${ }^{\circledR} \operatorname{SDR}^{\mathrm{TM}}$. Group 3: Post Cementation and reconstruction with Prime \& Bond NT + Self cure Activator and Core-X-flow core-build-up (Denstply, DeTrey, Konstanz, Germany). The standarized cores measure were: $6 \mathrm{~mm}$ diameter base, $5 \mathrm{~mm}$ diameter closed-end and $5 \mathrm{~mm}$ height. The push-out test was performed in a universal testing machine until fracture. The data were analyzed by two-way ANOVA and Tukey post hoc test at a significance level 5\%.

Root-filled mandibular premolars restored with direct composite with fiber post by different restoration technique, had similar fracture resistance under static loading.

Although similar fracture resistance results were obtained, Core-build-up materials showed better results compared with other techniques being the most recomended technique.

\section{OD31}

\section{Marginal sealing of bulk-fill composite at dentin cervical margin after thermal cycling: evaluation by OCT}

Gabriele Ventura ${ }^{1}$, Allegra Comba ${ }^{1}$, Anna Luganini $^{3}$, Mario Alovisi ${ }^{1}$, Camilla Alovisi $^{2}$, Elio Berutti ${ }^{1}$, Nicola Scotti ${ }^{1}$

${ }^{1}$ University of Turin, Department of Surgical Sciences, Dental School, Piemonte, Italy, ${ }^{2}$ University of Turin, Department of Surgical Sciences, Clinica Oculistica, Piemonte, Italy, ${ }^{3}$ Department of Life Sciences and Systems Biology, University of Turin, Piemonte, Italy

Objectives: The aim of this in vitro study was a non-invasive evaluation of the marginal sealing to dentin cervical margin of second class cavities after thermal aging using the optical coherence tomography (OCT). The null hypothesis is that there is no difference in marginal sealing between composites of different viscosity.

Methods: 8 intact upper premolars, extracted for periodontal reasons, were selected. A mesio-occluso-distal cavity with cervical margins placed $1 \mathrm{~mm}$ below the CEJ was performed on each tooth. Samples were then all treated with Scotchbond Universal in etch-and-dry technique. Restoration was performed as follow: a $1 \mathrm{~mm}$ horizontal layer of bulk-fill composite (Venus Bulk-Fill, Heraeus) was placed over only the mesio-cervical cavity floor and cured for $20 \mathrm{sec}$. Then composite restoration of the cavity was performed with oblique layering of nanohybrid resin composite (Clearfill ES-2, Kuraray). OCT ('Spectralis', Heidelberg-Engineering, Germany) imaging was performed after $24 \mathrm{~h}$ and 2000 thermocycles. Selected samples were sectioned for interfacial observation by confocal laser scanning microscope (CLSM). Marginal adaptation (percentage) was analyzed on 20 B-scans through each sample. Images were analyzed with the software ImageJ to assess the percentage of marginal gap between composite and cervical margin. Data were statistically analyzed with ANOVA test and significance was set for $\mathrm{p}<0.05$.

Results: Statistical analysis showed any differences in marginal adaptation obtained with Venus Bulk-Fill and Clearfill. Thermal cycling significantly affected marginal integrity of tested composites $(p=0.001)$. CLSM closely confirmed OCT findings in all samples.

Conclusion: The null hypothesis is accepted. Thermal aging significantly affected sealing of composites on dentin margin.

\section{OD32}

Comparison of silorane and conventional composites for water sorption and mechanical properties with adhesive coating

Burak Gumustas $^{1}$, Begum Guray Efes ${ }^{2}$

${ }^{T}$ Medipol University, Istanbul, Turkey, ${ }^{2}$ Istanbul University, Istanbul, Turkey

The purpose of this in vitro study was to determine the influence of water sorption on composite resins (3M Siloran, Kerr Sonicfill, $3 \mathrm{M}$ Z550, GC Kalore) with or without adhesive coating and its effects on flexural strength, surface hardness, surface roughness. Eight groups each containing ten discs $(15 \mathrm{~mm}$ diameter and $1 \mathrm{~mm}$ height) and bars $(25 \mathrm{~mm} \times 2 \mathrm{~mm} \times 2 \mathrm{~mm})$ made of each composite resin were made according to the manufacturer's instructions. Adhesive coating were applied to the last four group by dentin adhesive (3M Singlebond or 3M Silorane AS ). All the composites kept at $37^{\circ} \mathrm{C}$ for 24 hours and then measured for water sorption, flexural strength, surface hardness, surface roughness. At the second phase of the study, discs and bars were immersed into water for 90 days. Then they were thermocycled for 10000 times and remeasured. Data were subjected to analysis of Oneway ANOVA and Paired sample $t$ test $(\mathrm{P}>0 \cdot 05)$.Different organic matrix types of the composites caused different water sorption levels. The least water sorption levels were observed at Silorane composite groups. The least solubility levels were observed at Silorane and SonicFill composite groups. The highest levels of flexural strength and surface hardness were observed in SonicFill composite groups. $(p>0.05)$.Within the limitation of this study, the water sorption seem to affect the mechanical properties of the resin composite. Adhesive coating has a limited water sorption reducement in composite resin. 


\section{OD33}

In vitro evaluation of the bond strength of indirect composite restorative material to bleached enamel

Isil Dogruer $^{1}$, Can Dorter ${ }^{2}$

${ }^{T}$ Istanbul Yeni Yuzyil University, Istanbul, Turkey, ${ }^{2}$ Istanbul University, Istanbul, Turkey

Although the bleaching agent widely used clinically for a long time, there are some question marks about the effects of the dental bonding and restorative materials. The objective of this study was to investigate the effects of different concentrated bleaching methods on microtensile bond strength of tooth coloured indirect restorations. 20 sound extracted human third molars without any anomaly or restorations were used. Tooth seperated into two parts from mesiodistal direction. One surface was used as a experiment group, other surface was used as a control group. After bleaching experiment group with $40 \%$ Hydrogen Peroxide (HP), 10\% Carbamide peroxide (CP) and were stored for 14 days, total-etch (Kerr Optibond Solo) and self-etch (Kerr Optibond All-in-one) dentine adhesives, luting cement (Nexus 3) and $5 \mathrm{~mm}$. dimension tooth coloured indirect restoration blocks (GC Gradia Indirect) was applied on bleached enamel and unbleached enamel respectively. Microtensile bond strength tests were performed using microtensile test machine at $1 \mathrm{~mm} / \mathrm{min}$ speed until failure. The microtensile value was recorded as $\mathrm{mPa}$. Multiple comparisons were done by the One-way Anova test and Students $t$ test. Signifance evaluated as $p<0.05$. In both total-etch and self-etch groups, the microtensile bond strength values of HP and CP applied experiment group were significantly higher than control group $(\mathrm{p}<0.01)$. There were no statistically significant differences between HP and $\mathrm{CP}$ experiment groups in both total-etch and self-etch groups $(p>0.05)$. Within the limitation of this study, increasing degrees of porosity in enamel with bleaching agents, it's considered to improve adherence of the resin infiltration.

\section{OD34}

Effect of bulk thickness on microhardness of different bulk fill resin composites

Ugur Erdemir ${ }^{1}$, Emre Ozel ${ }^{2}$, Fulya Topcu ${ }^{3}$, Murat Tiryaki ${ }^{1}$, Elif Aybala Oktay $^{3}$, Miyiddin Saypullaev ${ }^{\top}$

${ }^{1}$ Department of Operative Dentistry, Istanbul University Faculty of Dentistry, Istanbul, Turkey, ${ }^{2}$ Department of Restorative Dentistry, Faculty of Dentistry, University of Kocaeli, Kocaeli, Turkey, ${ }^{3}$ Department of Endodontics and Conservative Dentistry, Gulhane Military Medical Academy, Ankara, Turkey

Objectives: The purpose of this study was to investigate the effect of bulk thickness on top and bottom vickers microhardness values (VHN) of high viscosity bulk fill resin composites.

Materials and Methods: Three bulk-fill materials (SonicFill, Filtek Bulk Fill and Tetric N-Ceram Bulk Fill) were evaluated. Seven specimens ( $\mathrm{n}=$ 7) from each material in different thickness (2,- and- $4 \mathrm{~mm}$ ) were prepared by using a stainless steel mold which is $8 \mathrm{~mm}$ in diameter. Tetric $\mathrm{N}$-Ceram Bulk specimens were light-cured for 20 seconds while SonicFill ve Filtek Bulk Fill were polimerized for 40 seconds with a QTH lamp at $850 \mathrm{~mW} /$ $\mathrm{cm}^{2}$ in one direction. All specimens were stored at $37^{\circ} \mathrm{C}$ for $24 \mathrm{~h}$, before hardness measurement. The microhardness measurements for top and bottom surfaces were performed using a digital vickers microhardness tester (load 200g; dwell time 30 seconds). Three indentations for both surfaces were recorded near the middle area on each specimens and avaraged. Data were statistically analyzed using Kruskal Wallis test and Tukey HSD post-hoc test $(\alpha=0.05)$.

Results: SonicFill exhibited the highest VHN for both 2- and $4 \mathrm{~mm}$ thickness and for upper and bottom surfaces $(p<0,001)$ while Filtek Bulk Fill the lowest $(\mathrm{p}<0.05)$. Bottom surface VHN values were significantly decreased with increasing bulk thickness $(\mathrm{p}<0.05)$. At $2 \mathrm{~mm}$ depth, all materials achieved at least $\% 80$ of their maximum VHN, but at the $4 \mathrm{~mm}$ depth any of the tested materials were achieved these results.

Conclusion: An increase in bulk tickness has resulted significantly decreased bottom VHN values irrespective of the bulf fill composite.

\section{OD35}

Sealing performance of resin composite to pulp chamber floor: evaluation by Optical Coherence Tomography

$\underline{\text { Alessandro Boaglio }}^{1}$, Allegra Comba ${ }^{1},{\text { Andrea } \text { Coscio }^{1}, \text { Mario Alovisi }}^{1}$, Camilla Alovisi ${ }^{2}$, Elio Berutti ${ }^{1}$, Nicola Scotti ${ }^{1}$

${ }^{1}$ University of Turin, Department of Surgical Sciences, Dental School, Italy, Turin, Italy, ${ }^{2}$ University of Turin, Department of Surgical Sciences, Clinica Oculistica, Italy, Turin, Italy

Objectives: The aim of this in vitro study was a non-invasive evaluation of the composite resin adaptation to cavity floor in post-endodontic restorations using the optical coherence tomography (OCT). The null hypothesis is that there is no difference in sealing performance of the pulp chamber floor between resins of different viscosity.

Methods: 30 intact upper molars, extracted for periodontal reasons, were selected, endodontically treated and filled with gutta-percha. Excess of gutta-percha was entirely removed from the chamber floor and teeth were randomly divided into 3 groups $(n=10)$ according to the material used for the restoration: Group A: 0,5 mm of flowable composite (Venus BulkFill, Hereus-Kulzer) and stratification of nanohybrid composite (Clearfil Majesty ES-2, Kuraray); Group B: single apposition of flowable composite (Venus Bulk-Fill, Hereus-Kulzer); Group C: stratification of nanohybrid composite (Clearfil Majesty ES-2, Kuraray). Materials adaptation to the cavity was assessed with the OCT ('Spectralis', Heidelberg Engineering, Germany) and images were analysed with the software ImageJ to assess the percentage of marginal gap between composite and chamber floor. Collected data were statistically analyzed with the ANOVA test and significance was set for $\mathrm{p}<0.05$.

Results: Statistical analysis showed a significant better adaptation of flowable composites compared to the traditional packable nanohybrid composites $(\mathrm{p}<0,05)$. No differences were found between group A and B.

Conclusion: Within the limitations of an in vitro study we can affirm that the use of a thin layer of flowable composite is recommended as first increment in post-endodontic restorations. Further investigations are necessary to confirm these results.

\section{OD36}

Color of conventional glass ionomer restoratives compared to shade guides

Panagiotis Ntovas, Panagiotis Lagouvardos School of Dentistry, University of athens, Athens, Attica, Greece 
Purpose: Selecting the correct shade for glass-ionomer restorative materials can be a challenge in contemporary clinical dentistry. The present study compares the color of conventional glass ionomers to the corresponding tabs of shade guides.

Materials and Methods: Three conventional glass-ionomers (Equia Fill/GC_EQF, Riva/SDI_RIV, Ketac Fill plus/3M-ESPE_KTF) and one resin composite (Charisma classic/Heraeus-Kulzer_CCL) were selected. Four shades from each material were used(A1,A2,A3, A3.5) to make 20 discoid specimens of $3 \mathrm{~mm}$ (thickness) by $6 \mathrm{~mm}$ (diameter)from each material. Three different shade guides were also selected(Vita Lumin Vacuum/Vita-VLV, Vintage Halo/Shofu-VHS, Chromascop/ Ivoclar-Vivadent-CRO). Before color measurements, specimens were hydrated for $24 \mathrm{~h}$ in water of $37^{\circ}$ and a mean of three measurements was recorded for each specimen of material or guide. Measurements were recorded in the CIELAB color space using a portable contact colorimeter(Shade Eye/Shofu). Two way ANOVA tests were performed to estimate significant differences between material shades and corresponding tabs of the guides, in the primary and secondary color parameters $\left(\mathrm{L}^{*}, \mathrm{a}^{*}, \mathrm{~b}^{*}\right.$ and $\left.\Delta \mathrm{E}^{*}\right)$, at a significant level of 0.05 .

Results: $\Delta \mathrm{E}^{*}$ values were mostly above 3.3 and ranged from $2.3-16.2$ units for EQF,3.6-19 units for RIV and 2.9-17.7 units for KTF. Comparisons among shade guides showed also differences ranged from 6.3-11.5 for VLV,2.3-9.9 for SVH and 2.8-8.8 for CRO while $\Delta \mathrm{E}^{*}$ values for the resin CCL from glass-ionomers ranged from 10.0 to 19.0 units.Statistical analysis showed significant differences in $\Delta \mathrm{E}^{*}$ among materials and shade guides $(\mathrm{p}<0.05)$.

Conclusions: Glass-ionomer shades present significant differences from the corresponding shades in all guides. However the differences are higher with the VLC among guides and lower with EQF among brands.

\section{OD37}

The effect of different solutions on the shear bond strength of repaired leucite ceramics

Aylin Cilingir ${ }^{1}$, Meryem Gulce Subasi ${ }^{2}$, Sevgi Zorlu ${ }^{3}$, Magrur Kazak ${ }^{4}$, Didem Oner Ozdas ${ }^{3}$, Solen Gunal ${ }^{4}$

${ }^{I}$ Trakya University Faculty of Dentistry Department of Restorative Dentistry, Edirne, Turkey, ${ }^{2}$ Istanbul Aydin University Faculty of Dentistry Department of Prosthodontics, Istanbul, Turkey, ${ }^{3}$ Istanbul Aydin University Faculty of Dentistry Department of Pedodontics, Istanbul, Turkey, ${ }^{4}$ Istanbul Aydin University Faculty of Dentistry Department of Restorative Dentistry, Istanbul, Turkey

Objective: The aim of this study was to evaluate the effect of different solutions on the shear bond strength of repaired leucite ceramics.

Material and Methods: Ninety-six ceramic (IPS Empress CAD) specimens were prepared $(12 \times 12 \times 2 \mathrm{~mm})$. Ceramic surfaces were etched with hydrofluoric acid for 60 seconds. A ceramic repair kit (Ivoclar Vivadent); was applied to the surfaces of ceramics according to the manufacturer's instructions. Samples were randomly divided into 4 groups depending on the repaired composite type $(\mathrm{N}=24)$, (Tetric N Ceram, Filtek Z550, Clearfil Majesty Esthetic, Ælite Aesthetic Enamel). Composites used for repair applied in a special cylindrical mould $(5 \times 2 \mathrm{~mm})$. Composite resins were polymerized by using a LED curing light (Elipar). After polymerization, each specimen was stored in artificial saliva at $37{ }^{\circ} \mathrm{C}$ for $24 \mathrm{~h}$ in an oven (Star Dental). The ceramic-composite repaired specimens were randomly divided into three subgroups according to the solutions $(\mathrm{n}=8)$.

1. Pomegranate Flavored Mineral Water (Uludağ Frutti)

2. Salad dressing (balsamic vinegar, olive oil, lemon, pomegranate syrup, basil, salt, garlic)

3. Control (Distilled water)

After 24h storage, shear bond strength tests were performed with a universal testing machine (858 Mini Bionix) at a crosshead speed of $1 . \mathrm{mm} /$ min. Data were statistically analysed with ANOVA and Tukey HDS tests.

Result: Tetric Nceram showed the highest shear bond strength in control groups $(\mathrm{p}<0.01, \mathrm{p}<0.05)$. Tetric Nceram showed higher shear bond strength than Filtek Z550 in salad dressing group $(\mathrm{p}<0.05)$.

Conclusion: Different composites and solutions have influence on the shear bond strength of repaired ceramics.

\section{OD38}

Effect of pre-treatment of hybrid CAD-CAM blocks on shear bond strength with self-adhering resin cements.

Christina Boutsiouki, Kosmas Tolidis, Paris Gerasimou Aristotle University of Thessaloniki, Thessaloniki, Greece

Introduction: Resinous cements are the material of choice for cementing indirect composite and ceramic restorations. However, there are no specific guidelines concerning pre-treatment of hybrid resin-ceramic CAD-CAM blocks. Purpose of the study was to test the effect of CAD-CAM block surface pre-treatment on the shear bond with self-adhering resinous cements.

Materials: 24 groups $(n=10)$ were formed according to i) type of block [Lava Ultimate,3M-ESPE(L) or Shofu Experimental Block,Shofu (S)], ii) type of cement [RelyX Unicem,3M-ESPE (R) or BeautiCem SA,Shofu (B)] and iii) surface pretreatment [none (CL), sandblasting (S), tribochemical coating (Cojet,3M-ESPE) (C), silane (SL), hydrofluoric acid + silane $(\mathrm{HF}+\mathrm{SL})$, Scotchbond Universal Bonding Agent,3M-ESPE (U)]. Specimens were manufactured with a circular bonding area of 16,61 $\mathrm{mm} 2$. Standardised Ultratester machine (Ultradent) was used at a crosshead speed of $0.5 \mathrm{~mm} / \mathrm{min}$.

Results: Shear bond strength results in $\mathrm{MPa}($ mean/SD) are: LR-CL (12.89/3.54), LR-S (15.86/4.96), LR-C (17.2/1.46), LR-SL (14.23/ 3.67), LR-HF + SL (13.13/2.14), LR-U (17.88/5.61), SR-CL (4.91/ 2.01), SR-S (12.83/1.26), SR-C (11.45/4.23), SR-SL (13.76/4.77), SR-HF + SL (20.11/6.95), SR-U (13.56/4.9), LB-CL (12.37/2.14), LB-S (17.09/1.89), LB-C (20.47/1.59), LB-SL (10.98/1.01), LBHF + SL (15.37/2.28), LB-U (16.39/1.53), SB-CL (4.19/1.51), SB-S (13.48/1.31), SB-C (14/1.28), SB-SL (9.15/1.1), SB-HF + SL (19.98/5.98), SB-U (9.48/1.16). In total, more cohesive failures in blocks $(51 \%)$ are recorded, followed by adhesive $(37.5 \%)$. Statistically significant differences are exhibited in comparison to the control groups for block $\mathrm{S}$.

Conclusions: When bonding to hybrid CAD-CAM blocks with selfadhering resinous cements, it is advised to pre-treat the block surface as follows: with S, C or U when bonding to Lava blocks and with $\mathrm{HF}+\mathrm{SL}$ when bonding to Shofu Experimental blocks, regardless the cement. 


\section{OD39}

Have French dentists implemented recent concepts in cariology into everyday practice for occlusal caries lesions?

$\underline{\text { Sophie Doméjean }}^{1}$, Stéphanie Léger ${ }^{2}$, Marie Maltrait ${ }^{1}$, Ivar Espelid ${ }^{3}$, Anna Tveit $^{3}$, Stéphanie Tubert-Jeannin ${ }^{1}$

${ }^{1}$ UFR d'Odontologie, EA 4847, Centre de Recherche en Odontologie Clinique, Clermont-Ferrand, France, ${ }^{2}$ Univ Blaise Pascal, Département de Mathématiques, Clermont-Ferrand, France, ${ }^{3}$ Faculty of Dentistry, University of Oslo, Oslo, Norway

Objective: Management decisions for occlusal carious lesions were investigated in 2012 using a questionnaire survey among French dentists. Restorative thresholds and management of questionable lesions were described and compared to guidelines from recent scientific literature.

Methods: A questionnaire developed by Espelid et al. was sent by mail to a random sample of 2,000 French dentists in 2012.

Results: Response rate was $41.9 \%$ (838 respondents).

Restorative Threshold: $39.3 \%$ of the respondents would place a restoration for a lesion confined to enamel, $54.7 \%$ for a lesion at the enameldentin junction and $6.0 \%$ for a lesion in the outer $1 / 3$ of dentin or deeper.

Diagnosis: based on a photograph showing an occlusal surface, $40.3 \%$ of the respondents diagnosed an enamel lesion, $10.1 \%$ a dentinal lesion, $32.3 \%$ a sound surface while $14.8 \%$ were uncertain. The management strategies proposed were a non-invasive management, a minimally invasive restoration or a restoration comprising all pits and fissures for respectively $54.6 \%, 33.4 \%$ and $12.0 \%$ of the respondents for whom diagnosis was uncertain $(\mathrm{n}=114)$

The present results show that French dentists have not implemented the recent concepts of caries management into practice. Indeed, restorative strategies should be limited to the treatment of carious lesions reaching the middle $1 / 3$ of the dentine and/or with definitive cavitation in the overlying enamel. Moreover, evidence supports nowadays the use of non-invasive management of occlusal non-cavitated or questionable lesions.

Conclusion: More efforts are needed to enhance the dissemination, acceptance and use of the concepts of Minimal Intervention in cariology for French dentists.

\section{OD40}

Biocompatibility of a new fiber-reinforced composite: an in-vitro study

Lina Dimosiari, Fany Ziouti, Elisabeth Koulaouzidou

Aristotle University of Thessaloniki, Thessaloniki, Greece

Introduction: A new fiber-reinforced composite (GC everX Posterior ${ }^{\mathrm{TM}}$ ) was designed to be used as dentine replacement, in conjunction with a conventional composite layer used as enamel replacement. The material is proposed to prevent and stop crack propagation through the filling, via the reinforcement provided by the fibers and is marketed as an economic alternative for chair-side restoration of extensive cavities.
Materials and Methods: The purpose of this study was to determine the cytotoxicity of the new fiber-reinforced composite restorative material (GC everX Posterior ${ }^{\mathrm{TM}}$ ) against a fibroblastic cell line. MRC5 cells were grown as monolayer cultures at $37^{\circ} \mathrm{C}$ in an atmosphere containing $5 \% \mathrm{CO}_{2}$ and $100 \%$ relative humidity. The culture medium was Dulbecco's modified medium supplemented with $10 \%$ Fetal Bovine Serum and antibiotics. Extracts were obtained from $24 \mathrm{~h}$ and 10 day incubations of composite specimens in cell culture medium at $37^{\circ} \mathrm{C}$. The cells were exposed to serial dilutions of the original extracts for $24 \mathrm{~h}, 48 \mathrm{~h}$ and $72 \mathrm{~h}$. Complete medium was used as control. The cytotoxic effect was determined, using the modified colorimetric sulforhodamine B assay, in reference to controls. Statistical analysis was performed with ANOVA/ post-hoc tests at $\mathrm{p}=0.05$.

Results: $24 \mathrm{~h}$ and $10 \mathrm{~d}$ extracts from the tested composite reduced cell viability after $24 \mathrm{~h}, 48 \mathrm{~h}$ and $72 \mathrm{~h}$ of exposure and the effect was concentration- and time- dependent.

Conclusion: The tested fiber-reinforced composite showed a potency of reducing viability of cells in vitro

\section{OD41}

\section{Influence of DCC in ethanol-wet bonding technique on bond strength} to radicular dentin

Nicola Scotti ${ }^{1}$, Carlotta Giuliano ${ }^{1}$, Laura Sponzilli ${ }^{1}$, Annalisa Mazzoni ${ }^{2}$, Lorenzo Breschi ${ }^{2}$, Valeria Angeloni ${ }^{3}$

${ }^{1}$ University of Turin, Turin, Italy, ${ }^{2}$ University of Trieste, Trieste, Italy,

${ }^{3}$ University of Bologna, Bologna, Italy

Objective: The aim of this in vitro study was to evaluate the effect of a cross-linker agent (DCC) used in addition to ethanol in ethanol-wet bonding technique for fiber post cementation to radicular dentin. The null hypothesis tested was that DCC do not influence immediate fiber post bond strength when employed with ethanol.

Methods: 18 extracted intact single-root teeth were selected and endodontically treated. A $10 \mathrm{~mm}$ post-space was prepared with dedicated drills and etched with $37 \%$ phosphoric acid. Samples were randomly divided into 3 groups $(n=6)$ according to the dentin pre-treatment procedure: 1) water-wet bonding: post space irrigation with distilled water for $60 \mathrm{sec} ; 2$ ) ethanol-wet bonding: post space irrigation with ethanol for $60 \mathrm{sec} ; 3$ ) ethanol-wet bonding with DCC: post space irrigation with $0.5 \mathrm{M}$ DCC in ethanol for $60 \mathrm{sec}$. Adhesive (AllBond3, Bisco) was, than, applied following manufacturer instruction, and fiber posts were luted with DuoLink Universal (Bisco) and cured for $40 \mathrm{sec}$. To perform push-out test of the coronal and apical region, samples were cut in $1 \mathrm{~mm}$ thick slices and pushed until failure with an Instrom Machine. Results were statistically analyzed with ANOVA test, and statistical significance was set for $\mathrm{p}<0.05$.

Results: ANOVA showed that both regions had comparable results for group 2 and 3, while for water-wet bonding the coronal region performed significantly better $(p=0.0001)$. No differences were found between the three pre-treatment techniques considering the region.

Conclusions: The null hypothesis has to be accepted since DCC did not influence immediate bond strength to radicular dentin when applied with ethanol. 


\section{OD42}

\section{Assessment of the Accuracy and Repeatability of Shade Taking using Visual and Digital Shade Guides}

Eman Mottaleb, Wei- Ming Tay, Chris Louca, Albert Leung, Robert Blizard

Eastman Dental Institute, London, UK

Objective: To investigate differences in accuracy and repeatability of shade taking using VITA Classical ${ }^{\mathrm{TM}}$ (VC), VITA Linearguide 3D$\operatorname{Master}^{\mathrm{TM}}$ (LG) and VITA Easyshade Advance 4.0 $0^{\mathrm{TM}}$ (ESA) shade guides; and whether $3 \mathrm{D}$ shades can be converted to Classical without affecting mean accuracy.

Methods: 36 participants identified the shades of VC tabs using the VC and LG guides under standardised conditions. This study was repeated using the ESA. 3D shades were converted to Classical shades using four conversion tables.

Results: ESA had a greater mean accuracy than visual guides (VC and LG guides combined $)(F=10.95 \mathrm{p}=0.02)$. There were significant differences between the methods used in this study of recording accuracy $(\mathrm{F}$ $=3.03 \mathrm{p}=0.032$ ). Greater accuracy was seen with Classical than with converted 3D shades. There were significant variations of the mean accuracy results between ESA and visual guides. These variations depended on whether classical or 3D values were recorded, and on which $3 \mathrm{D}$ to Classical conversion table was applied $(\mathrm{F}=3.895 \mathrm{p}=0.11)$. Repeatability for ESA was better than for visual guides; ESA was 90\% (95\% CI 84\%, $95 \%)$ and the visual guides were $45 \%$ (95\% CI 36\%, 53\%).

Conclusions: In vitro ESA has greater accuracy and repeatability than VC or LG guides. The mean accuracy of the results was affected by converting 3D to Classical shades. These issues are very important to clinical dentistry. Further research is required to compile more consistent conversion tables between 3D and Classical shades. A wider range of ceramics and composites should be made available in 3D shades.

\section{OD43}

Resin infiltration of non-cavitated proximal caries lesions: a systematic review

Ermeline Daniel $^{1,2}$, Elodie Savard ${ }^{1,2}$, Roddy Kheng ${ }^{1,2}$, Elisabeth Dursun $^{1,2}$

${ }^{T}$ Paris Descartes University, Paris, France, ${ }^{2}$ A. Chenevier Hospital, Créteil, France

Introduction: To arrest non-cavitated proximal carious lesion progression, a new procedure has been recently developed: the infiltration of enamel micropores by a resinous material, to create the diffusion barrier inside the lesion, replacing lost mineral with resin. This technique is between the prevention therapy (remineralization) and the invasive restorative dentistry (even minimally). The aim of this study was to perform a systematic review of the published literature concerning the caries infiltration.

Methods: An electronic search on the PubMed database was attempted to identify all relevant in vitro, in situ and in vivo studies on the proximal caries infiltration and on the shear bond strength to resin-infiltrated enamel. Papers published in English were selected after a critical review of theirs titles, theirs abstracts and finally theirs full texts.
Results: The initial search found 184 eligible papers and finally 30 were included in this systematic review. The articles allowed to determine the justification of each protocol step, to optimize the resin penetration in the body of the carious lesions and inhibit its progression (the acid type and concentration; the resin type, duration and number of applications). Other articles allowed to discuss the opening possibility to broader indications (for cavitated lesions) and to underline some unstudied steps of the protocol.

Conclusion: The infiltration of subsurface lesions with low-viscous lightcuring resins is an extremely conservative approach. Only three clinical studies during one and three years were performed. This technique is very promising but has to prove itself in the long term.

\section{OD44}

The effect of a bulk-fill base on the clinical survival of direct posterior composite restorations

Colin McGuirk, Fariha Hussain, Brian Millar KCL, London, UK

Introduction: Direct composite restorations are increasingly popular for the restoration of posterior teeth. Efforts have been directed at simplifying composite placement techniques without compromising the degree of cure or polymerisation contraction stress. A flowable material (SDR, Dentsply) claims to allow bulk-placement and curing whilst minimising polymerisation contraction stress by utilising a flexible polymerisation process. The aim of this study was to compare composite restorations performed with and without an SDR base.

Methods and Materials: A retrospective analysis was performed to compare restorations placed by different techniques. Subjects in Group G received restorations placed according to conventional layering techniques using a nano-hybrid composite (G-aenial, GC). Subjects in Group $\mathrm{S}$ received a bulk-fill base using SDR (Dentsply) with a conventional composite outer shell (G-aenial, GC). Data regarding survival, mode of failure and post-operative sensitivity was recorded and analysed.

Results: In total 54 Group S restorations and 71 Group G restorations were followed for a minimum of 24 months. Group S had a $92.6 \%$ survival and Group G had $93 \%$. Group S was more prone to failure by tooth fracture $(\mathrm{p}=0.033)$. In both groups failure was more likely in larger cavities as measured by number of surfaces $(p<0.001)$ and the need for cuspal coverage $(\mathrm{p}=0.004)$.

Conclusion: The different techniques appear to provide similar levels of survival in the short term. The mode of failure differs between the groups; teeth restored with a bulk-fill SDR base experienced significantly more tooth fractures than the conventional composite group.

\section{Preventive Dentistry (PD)}

PD01

Calcium Therapy Preserves Periodontium and Dentition

Mark J. Manhart DDS, Srijita Sen-Crowdhry MD, Kim Truka DDS, Thomas B Steg DDS, James Rezac DDS, Derek Golding DDS, Suzan Hahn DDS 
Calcium Therapy Inst., Omaha NE 68114, USA

It is evident that Calcium Therapy consilience is quite high. It fits well with mediating the angiogenetesis upon periodontal tissue invasion and metastasis. Dentists have used this high-impact preventive protocol for half a century. This paper affirms the hard data: thousands of calcium treatments have rendered a more complete diagnosis, preserved granulation tissues, oral bone and the dentition.

Materials \& Methods: The calcium compounds utilized provide long-term, non-invasive, reliable clinical benefits in all dental fields. On-site and full-mouth timed-release calcium materials are administered to the oral cavity that control acute and chronic inflammations and bacterial colonization for days, weeks and months.

Results \& Discusion: When oral anaerobic bacteria become rampant, a dramatic breakdown of the oral cavity ensues. For over five decades nearly every patient with minor gingival infection to "hopeless" periodontosis has undergone Calcium Therapy. Within minutes the minimum bacterial concentrations and inflammatory markers show improvement without surgery or anesthesia. The intense in-office therapy can be sustained indefinitely, even for antibiotic sensitivity patients. The toxic invasion is "turned off" and oral tissues calmed. In one simple monthly application blood serum calcium levels elevate and persist for weeks and can be safely maintained for years.

Conclusions: While calcium material investigations remain of indiscriminate disinterest to dentistry, Calcium Therapy has replaced periodontal surgery completely. The direct-site therapy rapidly manifests tremendous control over inflammatory outbreaks. Its conservative practicality improves diagnostic protocol, revitalizes the process of wound healing, preserves both the peridontium and dentition, and does no harm while providing and supporting excellent dental restoration.

\section{PD02}

A novel hand-held reflectometer to quantify enamel erosion: correlation with surface microhardness and calcium release.

Thiago S. Carvalho, Tommy Baumann, Ekaterina Rakhmatullina, Adrian Lussi

University of Bern, Bern, Switzerland

This study aimed at assessing initial in vitro enamel erosion with a novel hand-held reflectometer, and to correlate the findings with standardized laboratory methods: surface microhardness and chemical analyses of calcium. A total of 120 human enamel specimens were ground, polished, and randomly distributed into 6 groups. Initially, we measured enamel surface reflection intensity (SRI) with the novel hand-held reflectometer, and surface microhardness $(\mathrm{SMH})$ with a Knoop hardness machine. The specimens were individually eroded $\left(10 \mathrm{ml} ; 1 \%\right.$ citric acid; $\left.\mathrm{pH} 3.6,25{ }^{\circ} \mathrm{C}\right)$ for $1,2,4,6,8$, or $10 \mathrm{~min}$ (20 specimens/group). After erosion, we measured final SRI and SMH, and calculated the loss of SRI and SMH in percentage. Total amount of calcium released by each specimen to the citric acid was also analyzed with an atomic absorption spectrometer. Kruskal-Wallis tests were used to analyze the effect of erosion time on SRI, SMH and calcium release, and Spearman's Rank correlation was performed between SRI and $\mathrm{SMH}$, as well as between SRI and calcium release. Longer erosion times caused greater loss of SRI $(p<0.001)$, greater loss of SMH $(\mathrm{p}<0.001)$, and greater calcium release $(\mathrm{p}<0.001)$. A significant correlation was observed between SRI and SMH $(\mathrm{r}=$ $0.79 ; \mathrm{p}<0.001)$, and between SRI and calcium release $(\mathrm{r}=0.70$; $\mathrm{p}<0.001)$. The moderate to strong correlation observed between SRI and the two standardized methods shows that the hand-held reflectometer can be considered as a potential tool to assess initial enamel erosion.

\section{PD03}

In Vitro Monitoring of the Erosion Progression by a New Optical Device

Julia Kozik, Thiago Saads Carvalho, Adrian Lussi

University of Berne, School of Dental Medicine, Berne, Switzerland

Aim: The main objective of this study was the validation of a recently developed optical device for the detection of different stages of erosion on enamel. Another goal was to assess if the optical device can measure the preventive effect of a tin-containing $\left(\mathrm{Sn}^{2+}\right.$-containing) solution on enamel erosion.

Material and methods: Enamel samples $(n=30)$ were placed in 3 different treatment solutions: 1)tap water (control group $\mathrm{C}, \mathrm{n}=10$ ), 2) $\mathrm{NaF}$-solution (group $\mathrm{F}, \mathrm{n}=10$ ) and 3 ) $\mathrm{Sn}^{2+}$-containing solution (group $\mathrm{SF}, \mathrm{n}=10$ ). Enamel erosion was induced by incubating the samples in $0.65 \%$ citric acid $(30 \mathrm{ml} ; \mathrm{pH}=3.6)$ for a total of $1,2,4,6$ and $8 \mathrm{~min}$. Surface reflection intensity (SRI) was measured before and after each erosive challenge. In addition to this optical method, erosion quantification was performed by surface microhardness (SMH) and calcium analyses.

Results: The optical assessment of erosion showed an exponential reduction of SRI throughout the whole experiment in all groups. The final percentage SRI values in group SF were significantly higher than in the other groups, showing significant protection in group SF. The SMH values revealed significant differences between all groups, where group $\mathrm{C}$ showed greatest loss of hardness and group SF the least loss of hardness. No significant difference was observed in the total amount of calcium release between the groups. Calcium release, however, showed a satisfactory negative correlation to SRI $\left(r^{2}=-0.61\right)$.

Conclusions: Monitoring of the SRI can be applied to quantify initial erosive stages. The $\mathrm{Sn}^{2+}$-containing solution presented better protection of enamel against erosion than the other groups.

\section{PD04}

Fluoride varnish versus tray application for head and neck cancer patients' caries prevention

Melanie Nasseripour ${ }^{1}$, Siham Khoulane ${ }^{1}$, Geraldine Lescaille ${ }^{1}$, MarieJosé Javelot

${ }^{1}$ La Pitié Salpêtrière Hospital, Paris, France, ${ }^{2}$ Paris 7 University, Paris, France

Introduction: Due to radiotherapy-induced caries, head and neck cancer patients have systematic fluoride application protocol using trays. Our study will evaluate the efficacy of fluoride varnish as an alternative to this protocol. 
Methods: The double-blinded randomized clinical trial at La Pitié Salpêtrière Hospital will include patients 18 years and older, diagnosed with head and neck epidermoid carcinoma and treated with external radiotherapy. The control group is treated with fluoride trays, the test group with varnish. The clinical and radiological follow up and fluoride renewal is done every 3 months for 24 months.

Results: Five of these patients were included in our preliminary study. Clinical and radiological exam were conducted at baseline, 3, 6, and 9 months. Fluoride varnish was used at each visit. The findings were imported into Excel and cariogram ${ }^{\circledR}$. Despite the established xerostomia no new caries developed at study intervals.

Conclusion: Our preliminary study confirms as in scientific litteratture that fluoride varnish application is clinically efficient, non patientcompliance dependent, applicable despite limited mouth opening, and non-irritating oral mucosa.

\section{PD05}

\section{A national pilot study comparing prevalence of caries measured with DMF vs ICDAS in Senegal}

\author{
Adjaratou Wakha AIDARA $^{1,2}$, Fatou LEYE-BENOIST ${ }^{1}$, Denis \\ BOURGEOIS \\ ${ }^{1}$ Cheikh Anta Diop University, Dakar, Senegal, ${ }^{2}$ Claude Bernard Lyon 1 \\ University, Lyon, France
}

Aim: This pilot study has for main objective to measure the applicability and the utility of ICDAS index in a context of prevention in developing countries. Dental caries prevalence was evaluated among schoolchildren using DMF (WHO basic method) vs. ICDAS index in Senegal.

Materials and method: A representative stratified random cluster sample of 677 primary and college schoolchildren aged 12 and 15 years was examined for caries prevalence. The clinical examination was conducted in two steps for each groups. The investigator proceeded at first to the reckoning of the number of teeth decayed (D), missing (M) or filled (F) according to the WHO basic method. Then, after cleaning and drying all teeth, a two-digit ICDAS code was used to record data at each dental surface.

Results: The caries prevalence (96\%) was higher than expected in Senegal. ICDAS index provides $43 \%$ moreover information than DMF. The need for prevention (ICDAS1: $66 \% / 72 \%$ and ICDAS2 :54\%/58\%) and interception (ICDAS3: $40 \% / 42 \%$ and ICDAS4: $31 \% / 33 \%$ ) were higher than the need of curative treatment (ICDAS5: $18 \% / 23 \%$ and ICDAS6: $27 \% / 33 \%$ ) respectively among primary and college schoolchildren.

Conclusion: Preventive programs are urgently needed in Senegal. It's necessary to lead epidemiological studies in other African countries for determining caries prevalence using the ICDAS criteria to harmonize oral health regional planning.

\section{PD06}

First Iberian Clinical Practice Guideline for the prevention and non-invasive treatment of dental caries

Elias Casals-Peidró $^{1,2}$, Mari Angeles García-Pereiro ${ }^{3,4}$

${ }^{T}$ Institut Català de la Salut, Granollers (Barcelona), Spain, ${ }^{2}$ Sociedad

Española de Epidemiología y Salud Pública Oral (SESPO), Madrid,
Spain, ${ }^{3}$ Badalona Serveis Assistencials, Badalona (Barcelona), Spain, ${ }^{4}$ Universitat de Barcelona, L'Hospitalet de Llobregat (Barcelona), Spain

Introduction: Health professionals recognize the difficulty of identifying the most appropriate care and recommendations to be given to their patients due to the growing body of literature demonstrating clinical success to prevent dental caries. A clinical practice guideline (CPG) is a useful tool in helping health professionals looking for a summary of evidencebased recommendations and activities in an schematic format.

Methods: The Spanish Society of Oral Epidemiology and Public Health (SESPO) brought together experts from Spain and Portugal to develop a CPG to promote the prevention and non-invasive treatment of dental caries according to the best scientific evidence available. Results: Firstly, other national CPG regarding dental caries were evaluated. Secondly, the most valuable research was identified in the form of clinical trials. Thirdly, its applicability to Spain and Portugal was evaluated. Two different caries risk levels were stablished (high/low) and two age categories (from 0 to 5 year old/from 6 years old). The GPC introduces to already available tools to assess caries risk (Cariogram, Caries Risk Semaphore, Cambra questionaires) using tree diagrams and to the diagnosis of caries lesions. One age and risk is stablished, recommendations are given regarding the use of self-applied fluoride and non-fluoride products and also which professional care should be given. All recommendations for high-risk groups are always additional to recommendations for low risk.

Conclusions: This guide stablishes recommendations for the prevention and non-invasive treatment of dental caries reflecting the current philosophy of monitoring caries disease throughout life.

\section{PD07}

The wettability of different adhesive systems in superficial and deep dentine

Derya Merve Halaçoglu, Duygu Tuncer, Zümrüt Ceren Salman, Neslihan Arhun

Baskent University, Ankara, Turkey

The aim was to determine the wettability of different types of adhesive systems in superficial and deep dentine.

Materials and Method: 24 molar teeth were cut in bucco-lingual direction to produce deep(remaining dentine thickness $1 \mathrm{~mm}$ far from the pulp) and superficial dentine(dentine layer adjacent to enamel) on buccal and lingual surfaces, respectively. Each half of the teeth were used(n $=48)$. Dentine specimens were ground wet with 600-grit silicon carbide abrasive paper and were divided into four $\operatorname{groups}(\mathrm{n}=12)$ : All-Bond-Universal(etch-and-rinse)(AU), All-Bond-Universal(self-etch)(AS), Clearfil-SE-Bond(selfetch)(CSE) and Single-Bond-2(etch-and-rinse)(SB). 3 consecutive contact angle measurements were obtained during the application of the adhesive systems: $1.3 \mu \mathrm{l}$ drop of distilled water on prepared dentin; 2.Droplet of the adhesive resin; 3.Distilled water after polymerization of the adhesive. Data were subjected to analysis of variance for repeated measures among the groups $(\mathrm{p}<0.05)$.

Results: There was no significant difference between deep and superficial dentin for all measurements $(p<0.05)$. In 2.measurement, CSE was significantly different from AS and AU in both dentin groups. In 3.measurement, CSE bond group was 
significantly different from other adhesive systems in both dentin groups( $\mathrm{p}<0.05)$. All of the adhesive systems except CSE increases the wettability of the bond after primer application but decreases the wettability of the distilled water after bond application $(p<0.05)$ reaching the first prepared state of the dentin in both dentin levels.

Conclusion: Adhesive system choice is not correlated with the depth of dentin; however different adhesives may affect the wettability of the dentin substrates.

\section{PD08}

\section{The Relation Between Caries Risk Levels and Salivary Immunoglob-} ulins Of Individuals Aged 21-23

\author{
Aysegul Kaya ${ }^{1}$, Tijen Pamir $^{1}$, Necil Kutukculer ${ }^{2}$ \\ ${ }^{T}$ Ege University School of Dentistry, Izmir/ Bornova, Turkey, ${ }^{2}$ Ege Uni- \\ versity School of Medicine, Izmir/ Bornova, Turkey
}

Purpose: This study aimed to identify high or low caries risk levels of the individual ages between 21-23 years and to associate their salivary IgA, $\operatorname{sg} \mathrm{A}$ and $\operatorname{IgG}$ with the caries risk.

Materials and Methods: Sampling frame of this study was composed of the students (ages between 21-23 years) from Ege University, Faculty of Agriculture, İzmir, Turkey. 87 (32 female, 55 male) participations out of 1960 volunteers orally examined were enrolled in the study. Cariogram was used in order to observe risk status of the volunteers and to illustrate the interaction of their caries related factors. CRT bacteria (Ivoclar/Vivadent) was used for determination of salivary mutans streptococci and lactobacillus while CRT buffer (Ivoclar/Vivadent) for buffer capacity. After identification of high or low levels of risk status, stimulated saliva of the participations was again collected and stored at $-70{ }^{\circ} \mathrm{C}$. A direct enzyme-linked immunosorbent assay (ELISA) method was used to measure IgA, sIgA and IgG levels. All data were statistically analyzed for the correlations.

Results: It was not found any correlation between the caries risk status of the participations and their salivary $\operatorname{sIgA}\left(r_{\mathrm{s}}=-0,209, \mathrm{p}\right.$ $=0,052)$ and $\operatorname{IgA}\left(r_{\mathrm{s}}=-0.206, \mathrm{p}=0.056\right)$. However, there was negative correlation between risk status and $\operatorname{IgG}\left(\mathrm{r}_{\mathrm{s}}=-0.277, \mathrm{p}<\right.$ $0.01)$.

Conclusion: Within the limitations of this study, it seems that other factors might be responsible from the caries process rather than local and general immunity. However, the relation between dental caries and IgG should be studied further.

Keywords: Immunoglobulins, caries, ELISA test

\section{PD09}

\section{Effect of loupe and head lamp on the visual caries detection using ICDAS}

Edi Jasarevic, Isabelle Hug, Klaus Neuhaus, Adrian Lussi

$\overline{\text { Department }}$ of Preventive, Restorative and Pediatric Dentistry, University of Bern, Bern, Switzerland

Aim: We determined the effect of an additional head lamp with white light on visual caries detection using ICDAS.
Materials and Methods: Nine examiners (4 students with one year and 5 students with two years of clinical experience) evaluated caries lesions in 28 extracted human teeth mounted in a dental phantom head.

Each examiner was asked to make 8 examinations: one using an operation lamp but without any visual aids, one with an additional head lamp with white light (LED), one with dental loupes $(2.5 \mathrm{x})$ and one with a combination of head lamp and loupes. Between each examination there was an interval of one week. The International Caries Detection and Assessment System (ICDAS) was used for caries detection. Photographs of each surface of the extracted teeth served as gold standard.

Results: At D1-threshold, sensitivity using loupe/lamp was significantly lower than without any visual aids (0.44 compared to 0.55$)$. Specificities and sensitivities on the D3-threshold were not influenced by any method $(0.52$ to 0.6 ). There was a trend of higher intraexaminer agreement when optical aids were used (from 0.57 with no visual aids to 0.74 with lamp and loupes).

Conclusion: Generally speaking, an additional headlamp with white light does not support caries detection. In addition, combination of white light and loupes may hamper caries detection.

\section{PD10}

Reproducibility of near-infrared light transillumination for approximal enamel caries: a clinical controlled trial

Fabian Jost ${ }^{1}$, Jan Kühnisch ${ }^{2}$, Adrian Lussi ${ }^{1}$, Klaus Neuhaus ${ }^{1}$ ${ }^{T}$ Department of Preventive, Restorative and Pediatric Dentistry, University of Bern, Bern, Switzerland, ${ }^{2}$ Department of Conservative Dentistry and Periodontology, Ludwig-Maximilians-University of Munich, Munich, Germany

Aim: To test the reproducibility of a near-infrared (NIR) transillumination device (DIAGNOcam, KaVo) for approximal enamel caries detection.

Materials \& Methods: Seventy unrestored approximal surfaces in 35 patients with permanent dentition (age $>14$ ) were included in this study. Initially, the patients underwent visual examination and bitewing radiographs (BW) were taken, if necessary. After receiving informed consent one interdental space with either an initial lesion and/or no caries was chosen. Both teeth of this selection were photographed with NIR transillumination and an orthodontic separation rubber was placed. The gold standard was visual-tactile caries detection, which was performed upon removing the rubber the next day. The tooth surfaces were examined by two examiners using the universal visual scoring system (UniViSS) with a surgical microscope at $6.4 \mathrm{X}$. Reproducibilities were calculated using weighted kappa (wK) statistics.

Results: Out of the 70 surfaces 31 (44\%) had no caries. The intra-rater agreement of both diagnostic methods presented good wK. Intra-rater agreement (BW/NIR) was $(0.62 / 0.68)$ for examiner 1 and $(0.79 / 0.62)$ for examiner 2, respectively. The inter-rater agreement with the $\mathrm{BW}$ (wK 0.62) showed a good correlation as well, while the wK value for NIR was only moderate $(0.51)$.

Conclusion: Generally speaking, agreement with NIR was worse compared to BW. The reason for this could be the missing calibration of the examiners and shadow artifacts in the margin region of the tooth in some NIR-pictures. They may be attributable to tooth morphology, crown angulation or width of the alveolar bone. 


\section{PD11}

Oral health status of non-phobic/dentally phobic individuals: Secondary analysis of the Adult Health Dental Survey

Ellie Heidari, Avi Banerjee, Tim Newton

King's College London, London, UK

Introduction: Dental anxiety is widespread across the United Kingdom and according to Adult Dental Health Survey (ADHS, 2009 ), $11 \%$ of the adult population have severe dental anxiety. Dentate people with dental anxiety present with poorer oral health and attend dentists irregularly. Dental anxiety is higher among those from the lower social classes and among females.

The aim of this study was to analyse the oral health status of patients with and without dental anxiety through secondary analysis of data from a National survey of the UK population. We will also describe the oral health's determinants and oral health related QOL and Oral Impacts on Daily Performance [OIDP]) in both groups.

Methods: Permission to access the data set of the ADHS was sought from UK discovery.

Descriptive statistics comparing individuals with severe levels of dental anxiety (MDAS score $>=19$ ) to those with moderate or mild dental anxiety, were calculated using SPSS v11.0

Results: There were more women (16.8\% [1023]) than men (7\% [344]) who reported dental phobia. Generally, people with dental phobia were unskilled workers, single with lower educational attainment. They also were irregular attendees, had less restored dentition, increased scores of Puss, Ulceration, Fistuale, Abscess (PUFA). However, they had the similar numbers of sound and missing teeth as participants without dental phobia. Interestingly, they reported better oral hygiene practises.

Conclusion: Patients with dental phobia have greater dental treatment needs. They perceive their oral health as poor which has an implication for their oral health related quality of life.

\section{PD12}

FoodForTeeth: A novel approach to diet diaries, caries risk and erosion risk

Prateek Biyani ${ }^{1}$, Jasneet Gulati ${ }^{2}$

${ }^{1}$ The University of Sheffield, Sheffield, UK, ${ }^{2}$ Guy's and St Thomas' NHS

Foundation Trust, London, UK

The Dental team plays an important role in educating patients about the impact of diet on oral health. The two major disease processes influenced by our diet are caries and dental erosion.

Advances in mobile technology have seen a modernisation of the way that information is shared and patient education is delivered in healthcare. This includes the use of applications (apps) that can be downloaded on to most mobile devices.

The FoodForTeeth app was designed as an oral health promotion tool with the modern patient in mind. The app has two major objectives:
1. To allow patients to record a diet diary, which they can share with their dentist to receive tailored dietary advice;

2. To allow patients to search a database of common foods and drinks to learn about their caries and erosion risk.

Diet diaries are an underutilised investigation in general dental practice. The app allows patients to easily record a diet diary, and is supplemented with instructions and advice to support the patient. FoodForTeeth aims to promote the use of the diet diary as a special investigation in patients who experience caries or dental erosion.

The app allows patients to search a user-friendly food and drink database, with caries risk and dental erosion risk expressed as traffic light indicators. The role of dietary carbohydrates in the patient experience of caries is just one part of a complex disease process, but the app aims to help patients make informed decisions about their diet and thereby promote oral health.

\section{PD13}

Design, build and validation of a realistic artificial mouth model for erosion testing.

Abubaker Qutieshat, Graham Chadwick, Andrew Mason University of Dundee, Dundee, UK

To facilitate the development of a realistic erosion testing regime, an in vitro artificial mouth model has been designed, built and validated. This work was sought to mimic the interaction of saliva and enamel during the process of consuming an erosive beverage. The model allows researchers to gather data using customizable experimental diets without the technical burden of dealing with a non-realistic regime. The design and build of the artificial mouth model along with its associated equipment and parameters are described. The device is designed on a fully adjustable multitask basis in which the operator can set several variables such as the desirable salivary kinematic behaviour, offensive beverage flow rate and volume of consumption. This, subsequently, allows the samples preloaded on the system to be tested for surface characteristics to determine the extent of erosion if any. The model also allows the resultant solution to be analysed for traces of calcium and phosphate ions. To validate the model, experimental diets were performed repeatedly using specimens prepared from ostrich eggshells, and compared to those obtained from extracted human teeth specimens for the same simulated experimental setup. The associated percentage error for surface ion loss and hardness were equal to or smaller than $0.8 \%$ and $1.0 \%$ respectively. The high degree of agreement and the consistency of results showed that our model is able to mimic realistic scenarios and is capable of producing reliable, reproducible and accurate outcomes. This capability allows further research to be conducted with focus on dental erosion prevention.

\section{PD14}

Dental sealants: comparison of different materials in a prospective, controlled, split-mouth clinical trial

Matteo Basso ${ }^{1}$, Manuel Goñe Benites ${ }^{1}$, Piyush Khandelwal ${ }^{2}$, Malek Turqui $^{1}$

${ }^{I}$ University of Milan, Galeazzi Orthopaedic Institute, Dental Clinic, Milan, Italy, ${ }^{2}$ GC Europe NV, Leuven, Belgium 
The purpose of this study is to compare the performance of a flowable composite resin (GradiaFlo, GC Corporation, Japan) with a glassionomer cement (Fuji Triage, GC Corporation, Japan), evaluating their preventive action on dental caries and mechanical strength after placement as fissure sealants. 41 patients aged less than 14 years were selected. They needed sealants on all 4 first molars, for a total of 164 seals. One side of the mouth was randomly assigned to composite group, and the other half to glassionomer group (split-mouth). Follow-ups were scheduled at 2, 4, $6,12,18,24$ months. Through magnifying loupes (Zeiss, 3.5x) and intraoral photographs the state of the seals (intact, partially lost, lost) and the presence of dental caries in sealed areas were evaluated. At 24 months, in the composite group, of the initial 82 sealants, 18 were found intact. During the study were detected 15 partially-lost composite sealants, and 15 lost. In the glassionomer group 17 were intact at 24 months, with 27 partially lost and 19 completely lost. It was detected the occurrence of one new caries for each group after 2 years. After statistical analysis, no difference is reported by the number of restorations that were completely lost for the 2 groups (T-test $p=0.23$ ). The glassionomer group has a greater tendency to partial loss $(p=0.008)$ while the composite group has a greater tendency in preservation of the integrity $(\mathrm{p}=0.02)$. Despite these differences, both materials appear equally effective in preventing tooth decay. 